\title{
COMPARISON OF SYSTEM IDENTIFICATION TECHNIQUES WITH FIELD VIBRATION DATA FOR STRUCTURAL HEALTH MONITORING OF BRIDGES
}

\author{
A thesis submitted to \\ the Faculty of Graduate Studies and Postdoctoral Affairs \\ in Partial Fulfillment of the requirements for the degree \\ Master of Applied Science \\ by
}

Muhammad Shamiur Rahman

Department of Civil and Environmental Engineering Carleton University

Ottawa-Carleton Institute of Civil and Environmental Engineering

May 2012

C2012 Muhammad Shamiur Rahman 
Library and Archives

Canada

Published Heritage

Branch

395 Wellington Street

Ottawa ON K1A ON4

Canada
Bibliothèque et

Archives Canada

Direction du

Patrimoine de l'édition

395 , rue Wellington

Ottawa ON K1A ON4

Canada
Your file Votre référence

ISBN: 978-0-494-91520-2

Our file Notre référence

ISBN: $978-0-494-91520-2$
NOTICE:

The author has granted a nonexclusive license allowing Library and Archives Canada to reproduce, publish, archive, preserve, conserve, communicate to the public by telecommunication or on the Internet, loan, distrbute and sell theses worldwide, for commercial or noncommercial purposes, in microform, paper, electronic and/or any other formats.

The author retains copyright ownership and moral rights in this thesis. Neither the thesis nor substantial extracts from it may be printed or otherwise reproduced without the author's permission.
AVIS:

L'auteur a accordé une licence non exclusive permettant à la Bibliothèque et Archives Canada de reproduire, publier, archiver, sauvegarder, conserver, transmettre au public par télécommunication ou par l'Internet, prêter, distribuer et vendre des thèses partout dans le monde, à des fins commerciales ou autres, sur support microforme, papier, électronique et/ou autres formats.

L'auteur conserve la propriété du droit d'auteur et des droits moraux qui protege cette thèse. $\mathrm{Ni}$ la thèse ni des extraits substantiels de celle-ci ne doivent être imprimés ou autrement reproduits sans son autorisation.
In compliance with the Canadian Privacy Act some supporting forms may have been removed from this thesis.

While these forms may be included in the document page count, their removal does not represent any loss of content from the thesis.
Conformément à la loi canadienne sur la protection de la vie privée, quelques formulaires secondaires ont été enlevés de cette thèse.

Bien que ces formulaires aient inclus dans la pagination, il n'y aura aucun contenu manquant. 


\section{Abstract}

Modal analysis together with finite element method (FEM) has evolved as a key technology in dynamic characterization of structures. The traditional modal analysis, known as Experimental Modal Analysis (EMA), identifies a modal model from the measured input forces applied to the test structure and the measured vibration response. More recently, system identification techniques has been developed to identify the modal model only from the vibration response of structure under its operational conditions. These techniques, referred as Operational Modal Analysis (OMA) or output-only modal analysis, take benefit of the available ambient excitation such as wind, traffic and turbulence. One of the prime motives for researchers to shift from the more established EMA to OMA is the problems associated with testing of large, complex systems such as civil structures like bridges, buildings, etc as it is very difficult to apply sufficient artificial excitation to large structures. Even under circumstances where it is possible to excite a large structure by controllable external sources, the corresponding high costs would likely make it not practical to employ EMA techniques for testing large structures. Another important advantage of OMA is that the structure can remain in its normal operating condition allowing identification of more realistic modal information for in-operation structures. Moreover, if the objective is continuous monitoring of structure, there is no alternative other than OMA. In OMA application, the role of the 
input signal is replaced by the assumption that the response is a realization of a stochastic process with unknown white noise as input. Due to the unavailability of input information in OMA, the parameter estimation process is significantly more complex than that in EMA. This thesis presents an in-depth discussion on existing system identification techniques and identifies the pros and cons of OMA. Four popular system identification algorithms namely Frequency Domain Decomposition (FDD), Eigensystem Realization Algorithm (ERA), Stochastic Subspace Identification (SSI) and recently developed right matrix fraction based Polyreference Least Square Complex Frequency Estimator ( $p$-LSCF) have been implemented on a software platform "Signal Processing Platform for Analysis of Structural Health" (SPPLASH), as part of an integrated monitoring system for continuous, real-time remote monitoring of the Confederation Bridge in Canada. In this thesis, system identification results by the these four algorithms applied to the processing of field measured ambient vibration data of the Confederation Bridge are compared on the basis of accuracy and efficiency of estimated modal parameters. 


\section{Acknowledgements}

I wish to express my gratitude to the people and institutions who made this thesis possible. Above all, to the Department of Civil Engineering at Carleton University for the facilities it offers for research, in particular to the Faculty of Graduate Studies and Post-doctoral Affairs for the Scholarship and teaching assistantship which provided me with the necessary funding to pursue graduate studies.

I owe special thanks to my thesis supervisor, Professor David T. Lau, who gave me the opportunity of participating in the Confederation Bridge project as a research assistant. It was a pleasure to work with him and learn from his experiences. I would like to thank my family for their encouragement and in terest in my studies. My deepest gratitude goes to Mitu for her support, patience, and encouragement throughout my studies.

Finally, I would like to mention my friends in Ottawa, especially Adam, Anton, Mitra, Mostafa, Amir, Amin, Mohammad and Waad. They made my study at Carleton University a rewarding and unforgettable experience. 


\section{Nomenclature}

$A_{c}, B_{c}, C_{c}, D_{c}$

$A_{d}, B_{d} C_{d} D_{d}$

$A, B$

$B_{2}$

$C_{a}, C_{v}$ and $C_{d}$

$C(\omega)$

$f(t)$

G

$G^{\text {ref }}$

$<g_{k}^{T}>$

$H(s)$

$h(k)$

I

$J$

$L_{c}^{T}$

$L_{m}^{T}$

$l$
Continuous-time state-space model

Discrete-time state-space model

Coefficient of matrix polynomial

Input location matrix (Finite element model)

Output location matrix for displacement, velocity

and acceleration

Singular value matrix of spectra

Excitation force at time $t$

Next-state output covariance matrix

Reduced next state-output covariance matrix

Operational reference factor for mode $k$

Transfer function matrix

Impulse response at time instant $k$

Identity matrix

Jacobian matrix

Participation matrix in state-space form

Participation matrix in modal form

Number of outputs 


$\begin{array}{ll}M, C, K & \text { Mass, damping and stiffness matrix for FE model } \\ m & \text { Number of inputs } \\ N_{b} & \text { Number of blocks } \\ n & \text { State-space model order } \\ n_{\max } & \text { Maximum state-space model order } \\ n_{2} & \text { Degrees of freedom in FE model } \\ n_{\alpha} & \text { AR model order } \\ n_{r} & \text { MA model order } \\ 0_{i} & \text { Observability matrix of order } i \\ q(t) & \text { Displacement vector at time } t \text { (FE model) } \\ R_{i} & \text { Output covariance matrix at lag time } i \\ R_{i}^{r e f} & \text { Reduced output covariance matrix at lag time } i \\ R_{u u} & \text { Input spectra for white noise } \\ S_{u u} & \text { Input spectra } \\ S_{y y} & \text { Output spectra } \\ T_{1 \mid i}^{r e f} & \text { Output covariance block Toeplitz matrix } \\ u(t) & \text { Input at time } t \\ u_{k} & \text { Mode shape vector } \\ V_{c} & \text { vit time instant } k \\ \left\{v_{k}\right\} & \\ v_{k} & \\ & \end{array}$




\begin{tabular}{|c|c|}
\hline $\boldsymbol{w}_{\boldsymbol{k}}$ & Process noise at time instant $k$ \\
\hline$X(s)$ & State in the Laplace domain \\
\hline$X(z)$ & State in the $\mathrm{z}$ domain \\
\hline$x(t)$ & State vector at time $t$ \\
\hline$x_{m}(t)$ & Modal state at time $t$ \\
\hline$x_{k}$ & State vector at time instant $k$ \\
\hline$Y(s)$ & Output in the Laplace domain \\
\hline$y(t)$ & Output at time $t$ \\
\hline$y_{k}$ & Output at time instant $k$ \\
\hline$\alpha, \beta$ & Rayleigh damping constants \\
\hline$\alpha$ & Matrix coefficient for PolyMAX method \\
\hline$\beta_{o}$ & PolyMAX matrix coefficient for outputo \\
\hline$\alpha_{i}$ & AR matrix parameters \\
\hline$\Gamma_{1}^{\text {ref }}$ & Reduced reverse stochastic controllability matrix \\
\hline $\boldsymbol{\theta}$ & Complex eigenvector of FE model \\
\hline $\boldsymbol{\theta}$ & Model parameter matrix for PolyMAX method \\
\hline$\Lambda$ & Diagonal matrix of $\mathrm{FE}$ eigenvalues \\
\hline$\Lambda_{c}$ & Diagonal matrix of continuous-time eigenvalues \\
\hline$\Lambda_{d}$ & Diagonal matrix of discrete-time eigenvalues \\
\hline$\lambda_{i}$ & Continuous time eigenvalues \\
\hline$\mu_{i}$ & Discrete time eigenvalue \\
\hline$\emptyset$ & Real eigenvector matrix of finite element model \\
\hline
\end{tabular}


$\omega$

$(\cdot)^{*}$

$(\cdot)^{\dagger}$

$(\cdot)^{H}$

(ी)

$\mathrm{E}[\cdot]$

$\mathcal{L}[\cdot]$

$\mathrm{Z}[\cdot]$

ARMA

ARX

CE

CMIF

ERA

EMA

FDD

IV

ITD

LSCE
Real eigenvector of finite element model

Damping ratio of mode $i$

Complex eigenvector matrix of state-space model

Diagonal matrix of circular eigenfrequencies, $\omega_{i}$

Circular frequency

Complex conjugate

Moore-Penrose pseudo-inverse

Complex conjugate transpose

Estimated value

Expected operator

Laplace transform

z-domain transform

Auto-Regressive Moving Average

Auto-Regressive with eXogenous

Complex exponential

Complex Mode Indication Function

Eigensystem Realization Algorithm

Experimental Modal Analysis

Frequency Domain Decomposition

Instrumental variable

Ibrahim Time Domain

Least Square Complex Exponential 
LSCF

ML

MPE

OMA

PEM

p-LSCE

p-LSCF

SSI-COV

SSI-Data

SVD

$\mathrm{ZOH}$
Least Square Complex Frequency Estimator

Maximum Likelihood

Modal Parameter Estimation

Operational Modal Analysis

Prediction Error Method

Polyreference LSCE

Polyreference LSCF (PolyMAX)

Covariance Driven Stochastic Subspace

Identification

Data-Driven Stochastic Subspace Identification

Singular Value Decomposition

Zero-order Hold 


\section{Table of Contents}

Abstract ...........................................................................................................................1I

Acknowledgements.................................................................................................

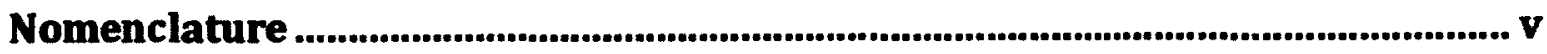

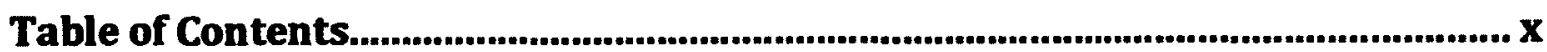

List of Tables ................................................................................................ Xiv

List of Figures............................................................................................................... XV

Chapter 1 - Introduction .................................................................................. 1

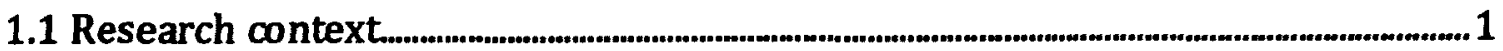

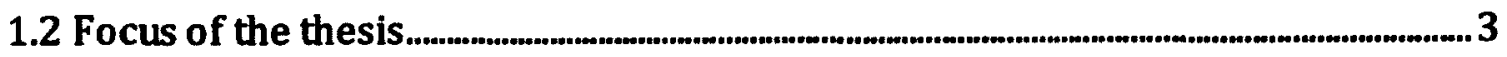

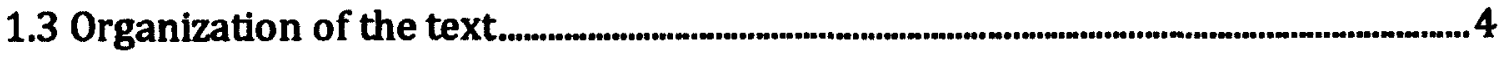

Chapter 2 - Models of vibrating structures .................................................5

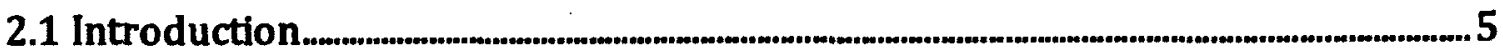

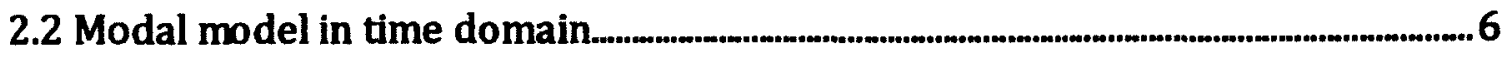

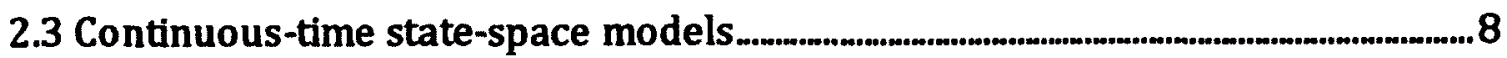

2.4 Discrete-time state-space models

2.5 Modal model in frequency-domain

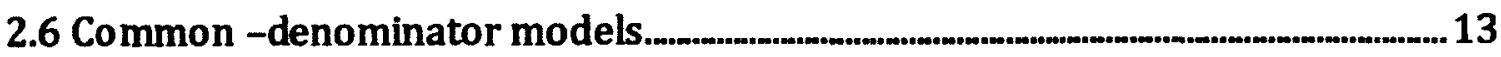


2.7 State-space models in the frequency-domain

2.8 Matrix fraction polynomial models

2.8.1 Left matrix fraction description (LMFD) models ........................................................... 16

2.8.2 Right matrix fraction description (RMFD) models............................................... 18

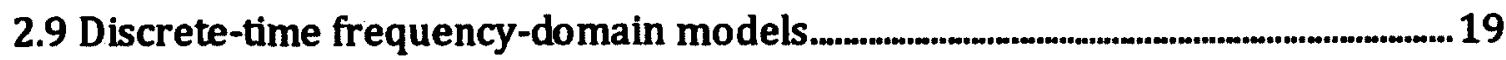

2.10 Primary identification data ........................................................................................................21

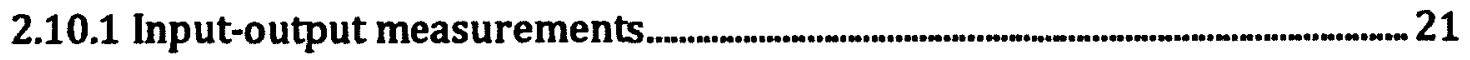

2.10.2 Output-only measurements: stochastic approach................................................ 22

2.11 Conclusions

Chapter 3 - Stochastic System Identification ..........................................................28

3.1 Introduction

3.2 A literature review of EMA algorithms .................................................................................... 29

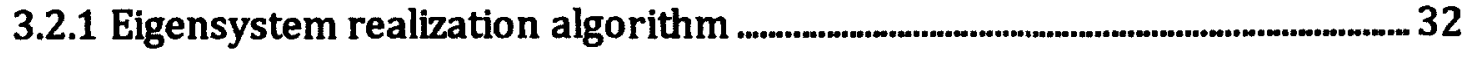

3.3 OMA algorithms

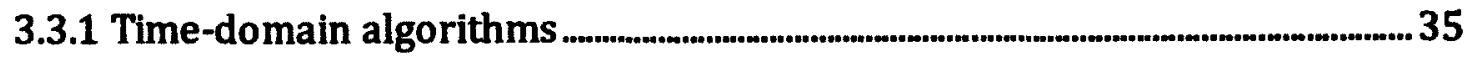

3.3.2 Spatial-domain algorithms

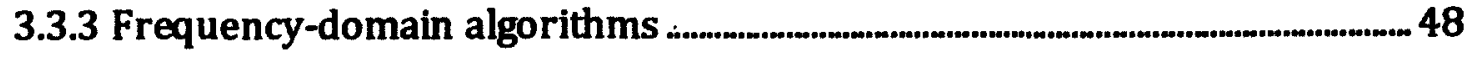

3.4 OMA associated data processing

Chapter 4 - Implementation of OMA: Pros and Cons.............................................66

4.1 Introduction

4.2 OMA applications

4.3 Major issues of OMA 


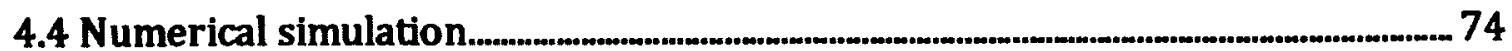

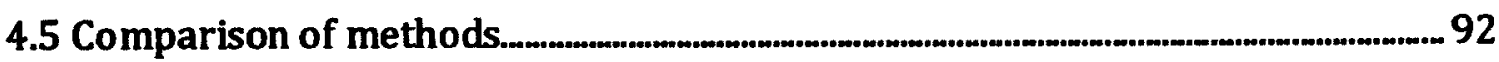

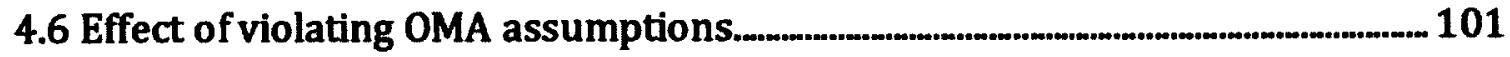

4.7 Damping estimate in OMA application

4.7.1 Cyclic averaging

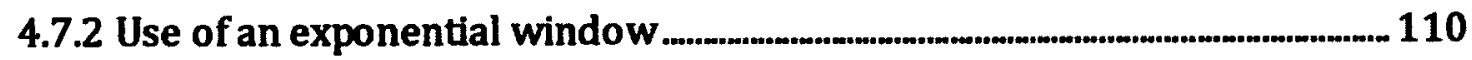

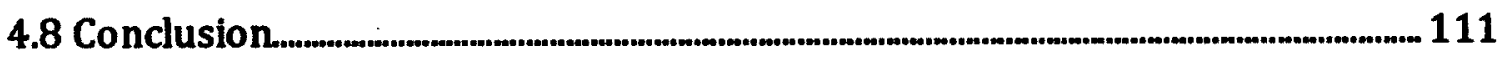

Chapter 5 - Application to Civil Structures........................................................ 113

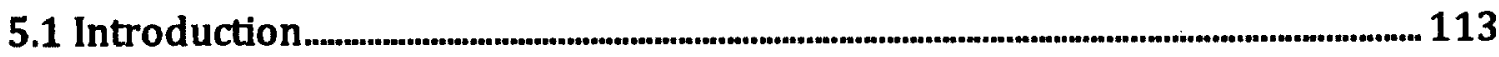

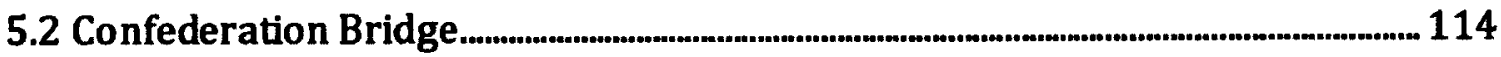

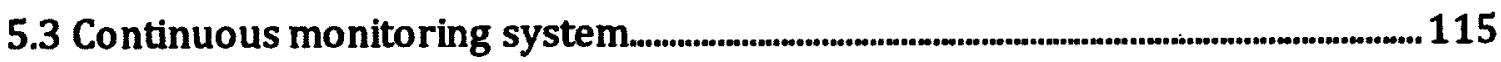

5.4 Software platform

5.5 Typical vibration behavior

5.6 Monitoring datasets

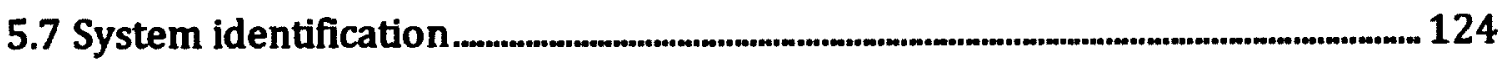

5.7.1 Stochastic subspace identification (SSI) $\ldots . . . \ldots \ldots$

5.7.2 Polyreference Weighted Least-Squares Complex Frequency Domain

Estimator (PolyMAX)

5.7.3 Eigensystem Realization Algorithm (ERA)

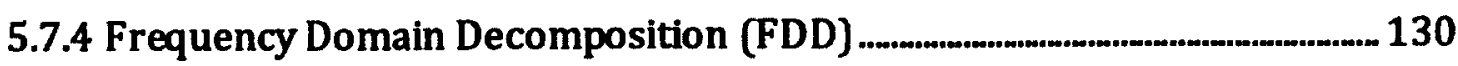

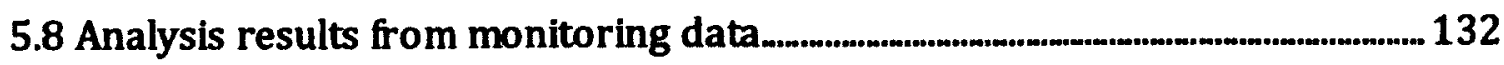

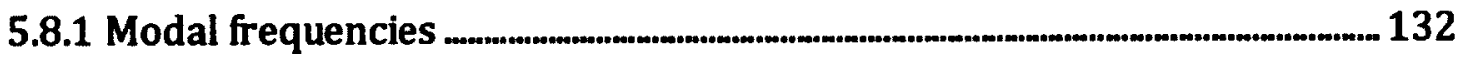




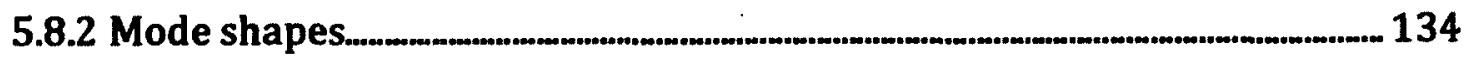

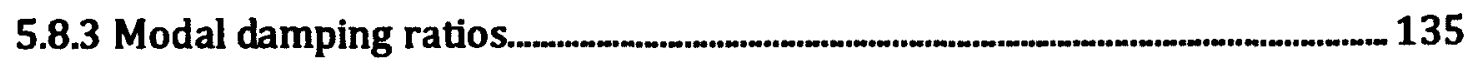

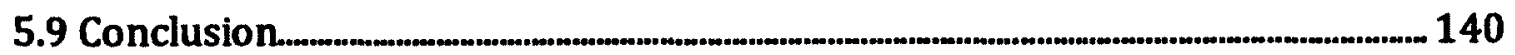

Chapter 6 - Conclusions and Recommendations for Future Work............... 142

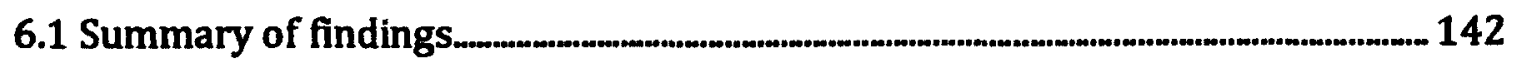

6.2 Future work

References ................................................................................................................................ 147 


\section{List of Tables}

Table 4-1: Modal frequencies and damping of analytical 8 DOF system ..............................75

Table 4-2: Modal parameters estimated using different OMA algorithms ...................... 92

Table 4-3: Modal parameter estimation results from 100 Monte-Carlo simulations.

The relative bias and relative std. deviations are given................................. 100

Table 4-4: Effect of correlated force on damping estimate ............................................................ 106

Table 4-5: Effect of cyclic averaging

Table 4-6: Effect of correlation lag time on damping estimate.................................................. 111

Table 5-1: Summary of modal frequencies identified by 4 algorithms and comparison to expected design values from finite element models

Table 5-2: Summary of modal damping ratios and Modal Assurance Criterion values for vibration modes retrieved by 4 system identification algorithms 


\section{List of Figures}

Figure 3-1: Stabilization diagram based on p-LSCF estimator with $\alpha_{1}=I_{r}$ constraint

Figure 3-2: Stabilization diagram based on p-LSCF estimator with $\alpha_{n+1}=I_{r}$ constraint

Figure 3-3: Schematic of positive power spectra estimation ...................................................... 65

Figure 4-1: Simulated 8-dof system.............................................................................................................74

Figure 4-2: Part of the first white input signal.................................................................................76

Figure 4-3: Part of the first output signal..................................................................................... 76

Figure 4-4: Part of the impulse response at 1 st DOF.................................................................77

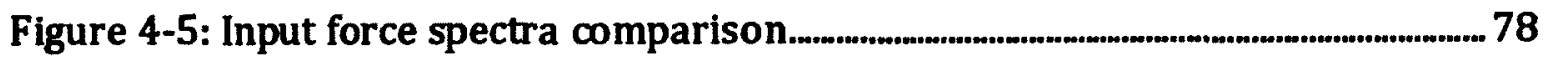

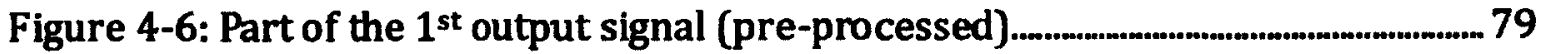

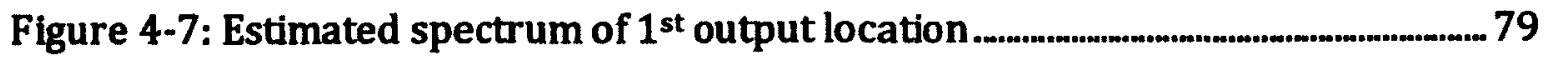

Figure 4-8: Estimated output covariance of $1^{\text {st }}$ output location..........................................80

Figure 4-9: $1^{\text {st }}$ four singular values obtained by SVD of the full spectrum matrix......81

Figure 4-10: Identified frequencies from pick picking of singular value........................... 82

Figure 4-11: MAC plots for FDD algorithm (with the analytical mode shapes)..............82

Figure 4-12: Singular values of the block Hankel matrix......................................................... 84

Figure 4-13: MAC plot between ERA identified modes and true modes.............................. 85 
Figure 4-14: Stabilization diagram for SSI method

Figure 4-15: Synthesized auto spectra of reference sensor 2

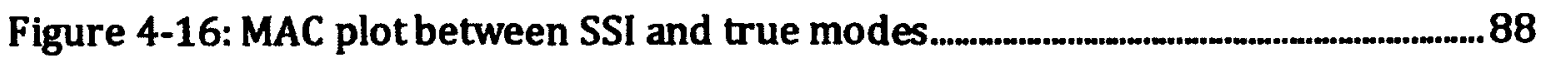

Figure 4-17: Clear stabilization diagram for PolyMAX method..............................................99

Figure 4-18: MAC plot between PolyMAX identified modes and theoretical modes........

Figure 4-19: Synthesized auto spectra for reference sensor 2 ................................................ 91

Figure 4-20: MAC plot for one run (PolyMAX)......................................................................96

Figure 4-21: MAC plot for same run (PolyMAX) …............................................................................96

Figure 4-22: Normalized frequency estimate from 100 Monte-Carlo simulations ...........

Figure 4-23: Normalized damping estimate from 100 Monte-Carlo simulations

.98

Figure 4-24: Mode shape estimation results from 100 Monte-Carlo simulations

Figure 4-25: SSI stabilization diagram in the presence of harmonics 102

Figure 4-26: PolyMAX stabilization diagram in the presence of harmonics ............... 103

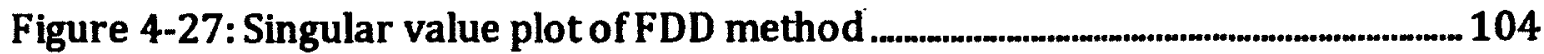

Figure 4-28: Comparison of force spectrum in case of partially correlated forces. 105

Figure 4-29: Effect of cyclic averaging for $1^{\text {st }}$ output location........................................... 109

Figure 5-1: Typical frame layout of Confederation Bridge....................................................... 115 
Figure 5-2: Locations of accelerometers in Confederation Bridge monitoring system

Figure 5-3: Average concrete temperature and average wind speed of 14 datasets

Figure 5-4: Stabilization diagram for SSI method................................................................. 126

Figure 5-5: Clear stabilization diagram for PolyMAX method.................................................. 128

Figure 5-6: Singular vale of block Hankel matrix ……............................................................ 130

Figure 5-7: Average singular values of the spectra matrices for both vertical and lateral direction

Figure 5-8(a): Comparison of experimental and analytical mode shapes between 0 $\mathrm{Hz}$ to $1 \mathrm{~Hz}$ 138

Figure 5-8(b): Comparison of some experimental and analytical mode shapes above $1 \mathrm{~Hz}$ 139 


\section{Chapter 1 - Introduction}

\subsection{Research context}

Modal analysis has evolved as a key technology in dynamic characterization of structures. Though initially developed as a simple technique for trouble shooting, it has become a standard approach in mechanical product development Continuously expanding its application area, modal analysis is today successfully applied in a wide variety of industries including aerospace, automotive, rotating machinery, civil structures such as buildings and bridges, etc.

Experimental modal analysis (EMA) identifies a modal model from the measured input forces applied to the test structure and the measured vibration responses. The extracted modal model defined by resonance frequency, damping ratio, mode shape and participation vector depends on the geometry, material properties and boundary conditions of the tested structure. The results from EMA application are used for a variety of purposes such as troubleshooting dynamics related problems, correlating and updating finite element model as well as simulation and prediction of structural modifications. Moreover, EMA has been effectively applied in the area 
of sensitivity analysis, force identification, structural damage detection and health monitoring, active vibration control, etc.

More recently, system identification techniques has been developed to identify the modal model only from the vibration response of structure under its operational conditions. These techniques, referred as operational modal analysis (OMA) or output-only modal analysis, take benefit of the available ambient excitation such as wind, traffic and turbulence. One of the prime motives for researchers to shift from the traditional and more established EMA to OMA is the problems associated with testing of large, complex systems such as civil structures like bridges, buildings, etc as it is almost impossible to provide sufficient artificial forced excitation to such huge structures. Even under circumstances when it is possible to excite the structure artificially, the corresponding high costs calls into question the acceptance of EMA in testing large structures. Another important advantage of OMA is that the structure can remain in its normal operating condition allowing identification of more realistic modal information for in-operation structures.

Nevertheless, the availability of technologies like Finite Element Method (FEM) has aided in changing the role of testing. However, there are serious limitations which restrict the use of FEM to complex, real life structures. These problems arise mostly due to the inability of FEM to properly model structural properties like damping, nonlinearity, boundary conditions as well as difficulties with the realization of in operation conditions. Especially there is no straight forward answer for damping information in the real life. So, these methodologies are accompanied by suitably 
designed experiments for acaurately determining the dynamic characteristics of the structure in consideration.

\subsection{Focus of the thesis}

The purpose of this study is to evaluate the efficiency and accuracy of different modal identification methods, particularly in the presence of high level of uncertainty and noise related to field measurement data. A total of 9 state-of-the-art stochastic system identification methods are critically reviewed and discussed in the thesis. They are divided into time-domain, spatial-domain and frequency-domain algorithms. Among these, 4 output-only system identification methods namely Frequency-Domain Decomposition (FDD), Eigensystem Realization Algorithm (ERA), Stochastic Subspace Identification (SSI) and the PolyMAX method have been compared also by means of a simulation study.

On the level of implementation, 3 modules related to the PolyMAX, Frequency Domain Decomposition (FDD) and Eigensystem Realization Algorithm (ERA) have been added to the existing Confederation Bridge monitoring software platform, Signal Processing PLatform for Analysis of Structural Health (SPPLASH).

Finally, a thorough analysis of the Confederation Bridge monitoring data by these output-only system identification techniques has been presented. 


\subsection{Organization of the text}

This thesis is divided into 6 chapters. The following provides a brief description of each chapter.

Chapter 1 presents motivation and objectives of the research. Chapter 2 discusses several models of vibrating structures that are useful in system identification. Instead of estimating the modal parameters directly from the measurements, the algorithms presented in chapter 3 identify first mathematical polynomial or state space models. These mathematical models then can be related to the modal parameters in a next step. Chapter 3 deals with stochastic system identification methods. These methods identify some of the models of Chapter 2 from output-only data. Chapter 3 also introduces OMA associated signal processing techniques. Chapter 4 focuses on the drawbacks of OMA and presents an up to data research work to overcome these limitations. Also, to clarify the theory and illustrate the practical use, the FDD, ERA, SSI and PolyMAX methods are applied to a simulation example. Furthermore, an attempt has been made to estimate damping correctly in OMA application using existing signal processing techniques. Chapter 5 presents the performance of 4 output-only system identification techniques with the Confederation Bridge monitoring data. Chapter 6 summarizes the conclusion of the thesis along with recommendations for future work. 


\section{Chapter 2 - Models of vibrating}

\section{structures}

\subsection{Introduction}

This chapter introduces several mathematical models that can be used to describe the vibrational behaviour of a structure with a limited number of parameters. From an engineering viewpoint the modal model of a structure offers the best physical understanding. However, as this model has high level of non-linearity in its parameters, most identification algorithms do not directly identify the modal parameters. Instead, the modal parameter estimation (MPE) methods introduced in the next chapter identify state space model, right matrix fraction description model and common denominator model, also known as scalar matrix fraction model from the experimental measurements. In the next sections, the relation between these models and the modal parameters both in time-domain and frequency-domain are discussed. The discussion is further subdivided into continuous-time and discretetime models. Additionally, a distinction is made between data driven and spectral 
function driven identification algorithms. Finally, the rationale behind using these models to identify modal parameters from both input-output measurements and from output-only measurements along with the concepts of an Experimental Modal Analysis and Operational Modal Analysis are introduced.

\subsection{Modal model in time domain}

The dynamic behaviour of a discrete mechanical system consisting of $\boldsymbol{n}_{2}$ masses connected through springs and dampers is described by the following matrix differential equation:

$$
M \ddot{q}(t)+C \dot{q}(t)+K q(t)=f(t)=B_{2} u(t)
$$

Here, $M, C, K \in \mathbb{R}^{n_{2} \times n_{2}}$ are the mass, damping and stiffness matrices; $q(t) \in \mathbb{R}^{n_{2}}$ is the displacement vector at continuous time $t$. The vector $f(t) \in \mathbb{R}^{n_{2}}$ is the excitation force. It is factorized into a matrix $B_{2} \in \mathbb{R}^{n_{2} \times m}$ that specifies the locations of the inputs and the vector $u(t) \in \mathbb{R}^{m}$ describing the $m$ inputs in time. Equation (2-1) is obtained as the finite element (FE) approximation of the system subdivided into only $n_{2}$ degree-of-freedom (DOF). From the geometry and material properties of the elements, the global mass matrix $M$ and stiffness matrix $K$ can be derived. The presence of the damping term is partially based on physical observation and partially on mathematical formulation of the physical phenomenon of energy dissipation in the dynamic behaviour of the system. In the formulation here, viscous damping is assumed to describe the observed decaying vibration behaviour. 
However due to the lack of identifiable or measurable material constants that govern the global damping behaviour of a structure, it is generally impossible to assemble the damping matrix $C$ in the same way as the mass and stiffness properties of the system. In most of the practical cases, damping is modeled as the special case of proportional damping. Equation (2-1) is used in the discussion here as a starting point to derive other models that are more suited in an experimental modeling context.

The computation of the eigenvalue and eigenvectors from Equation (2-1) is briefly studied next. Although these concepts are well known, it is useful to summarize the concepts that are relevant to this study here, both for the sake of completeness and for ease of referencing in later discussions in the subsequent chapters. The solution of the homogenous finite element model of the system without damping

$$
M \ddot{q}(t)+K q(t)=0
$$

has the following form:

$$
q(t)=\varphi_{i} e^{\lambda_{i} t}
$$

By inserting Equation (2-3) into Equation (2-2), a generalized eigenvalue problem is obtained:

$$
K \emptyset=M \emptyset \Omega^{2}
$$

where $\emptyset \in \mathbb{R}^{n_{2} \times n_{2}}$ contains the eigenvectors and $\Omega=\left[\because \omega_{i} \cdot\right] \in \mathbb{R}^{n_{2} \times n_{2}}$ is a diagonal matrix containing the eigen frequencies $\omega_{t}$. The eigenvectors diagonalize the mass and stiffness matrices. For the special case of proportional damping, the eigenvectors also diagonalize the damping matrix, $C$. A special case of proportional 
damping is also known as Rayleigh damping of which the damping matrix is a linear combination of the mass and stiffness matrices

$$
C=\alpha M+\beta K
$$

where $\alpha$ and $\beta$ are two scalar constants. As mentioned earlier in light of the high uncertainties of the many factors that can influence the damping behaviour of a structural system, it is often considered acceptable to adopt the proportional damping assumption in the finite element model of the system.

\subsection{Continuous-time state-space models}

\section{The state vector}

By casting the second order equation of motion (Equation (2-1) in first order form, an equation similar to the state equation from control theory is obtained. Reformulating Equation (2-1) as follows

$$
\left\{\begin{array}{l}
q(t) \\
\ddot{q}(t)
\end{array}\right\}=\left[\begin{array}{cc}
0 & I \\
-M^{-1} K & -M^{-1} C
\end{array}\right]\left\{\begin{array}{l}
q(t) \\
q(t)
\end{array}\right\}+\left[\begin{array}{c}
0 \\
M^{-1} B_{2}
\end{array}\right]\{u(t)\}
$$

results in a state-space formulation given by

$$
x(t)=A_{c} x(t)+B_{c} u(t)
$$

where $x(t)=\left(\begin{array}{l}q(t) \\ \dot{q}(t)\end{array}\right) \in \mathbf{R}^{n}$ is called the state-vector and $A_{c}$ is the state-space models in continuous-time. The related eigenvalue problem is given as follows

$$
A_{c} \Psi=\Psi \Lambda_{c}
$$


where $\Psi \in \mathbf{C}^{n x n}$ contains the $n=2 n_{2}$ complex eigenvectors and $\Lambda_{c} \in C^{n \times n}$ is a diagonal matrix containing the $n$ complex eigenvalue $\lambda_{i} . \Lambda_{c}$ and $\Psi$ have the following structure

$$
\Lambda_{c}=\left[\begin{array}{cc}
\Lambda & 0 \\
0 & \Lambda^{*}
\end{array}\right], \quad \Psi=\left[\begin{array}{cc}
\theta & \theta^{*} \\
\theta \Lambda & \theta^{*} \Lambda^{*}
\end{array}\right]
$$

where superindex, ${ }^{* * \prime}$ denotes complex conjugate. $\Lambda, \theta \in \mathbb{C}^{n_{2} x n_{2}}$ are the eigenvalues and eigenvectors of the original second order system. The complex eigenvalues $\lambda_{i}$ are written as:

$$
\lambda_{i}=\xi_{i} \omega_{i} \pm j \sqrt{1-\xi_{i}^{2}} \omega_{i}
$$

where $\xi_{i}$ is modal damping ratio corresponding to modal frequency $\omega_{i}$ for mode $i$ and $j$ is the imaginary unit defined as $j^{2}=-1$.

The observation equation

If the measurements are taken at $l$ locations the observation equation, in general, can be written as follows

$$
y(t)=C_{a} \ddot{q}(t)+C_{v} \dot{q}(t)+C_{d} q(t)
$$

where $y(t) \in \mathbb{R}^{l}$ are the outputs and $C_{a}, C_{v}, C_{d} \in \mathbf{R}^{l x_{2}}$ are the output location matrices for acceleration, velocity and displacement respectively. Using Equation (21) to eliminate $\ddot{q}(t)$ and with the definition of the state vector, Equation $(2-10)$ can be rewritten as follows

$$
y(t)=C_{c} x(t)+D_{c} u(t)
$$

where $C_{c} \in \mathbb{R}^{l \times n}$ is the output matrix and $D_{c} \in \mathbb{R}^{l \times m}$ is the direct transmission matrix. They are related to the finite element model matrices as follows: 


$$
C_{c}=\left(C_{d}-C_{a} M^{-1} K \quad C_{v}-C_{a} M^{-1} C\right), \quad D_{c}=C_{a} M^{-1} B_{2}
$$

The classical continuous-time state-space model is found by combining Equations (2-7) and (2-12). The order of the state space model $n$ is defined as the dimension of the state vector. The equation can now be used to compute the response $y(t)$ of the structure to a given input $u(t)$. The state space model is:

$$
\begin{aligned}
& x(t)=A_{c} x(t)+B_{c} u(t) \\
& y(t)=C_{c} x(t)+D_{c} u(t)
\end{aligned}
$$

By using the similarity transformation, it can be easily proved that there are infinite numbers of state-space representations that produce same input-output description. In order to better understand the relation to classical modal analysis, the state-space model is transformed to complex modal states $x_{m}(t) \in \mathbb{C}^{n}$ by using the special similarity transformation:

$$
x(t)=\Psi x_{m}(t)
$$

The modal state-space model is obtained by inserting the Equation (2-15) in Equation (2-14) and using the modal decomposition of $A_{c}$ (Equation 2-8) as follows

$$
\begin{gathered}
x_{m}(t)=\Lambda_{c} x_{m}(t)+L_{c}^{T} u(t) \\
y(t)=V_{c} x_{m}(t)+D_{c} u(t)
\end{gathered}
$$

where the following definition have been introduced:

$$
\begin{aligned}
& L_{c}^{T}=\Psi^{-1} B_{c} \\
& V_{c}=C_{c} \Psi
\end{aligned}
$$

The relations to notions from classical modal analysis are clear by taking a closer look at the modal input and output matrices $L_{c}^{T}, V_{c}$. By introducing the definition of $B_{c}$ (Equation 2-6) and $\Psi$, it can be easily shown that a row of modal input matrix, $L_{c}^{T}$ quantifies how "well" each degree of freedom is excited from a specific input 
location. Classically, the modal input matrix $L_{c}^{T}$ is called modal participation matrix and its rows are the modal participation factors. Similarly, by using the definition of $C_{c}$ (Equation 2-13) and $\Psi$ (Equation 2-9), the modal output matrix, $V_{c}$ can be described as the part of the mode shapes that can be observed from the data.

\subsection{Discrete-time state-space models}

Until now all equations were expressed in continuous time, whereas in the practice measurements are taken at discrete time instants. In order to fit models to measurements (i.e. system identification), these models need to be converted to discrete time. Furthermore, a discrete time model is needed for performing simulations in the absence of analytical solution and in most cases this is the reality. Typical for sampling of a continuous time equation is that certain behaviour of the time-dependent variables between two samples has to be assumed. A Zero-Order Hold ( $\mathrm{ZOH})$ assumption for instance, means that the input is piecewise constant over the sampling period. Under this assumption, by choosing a certain fixed sampling period $\Delta t$, the continuous-time state-space model Equation $(2-14)$ is converted to the discrete-time state-space model:

$$
\begin{aligned}
& x_{k+1}=A_{d} x_{k}+B_{d} u_{k} \\
& y_{k}=C_{d} x_{k}+D_{d} u_{k}
\end{aligned}
$$

The matrices $A_{d}, B_{d}, C_{d}, D_{d}$ are related to their continuous-time (Equation 2-14) counterparts as follows 


$$
\begin{gathered}
A_{d}=e^{A_{c} \Delta t}, \quad B_{d}=\int_{0}^{\Delta t} e^{A_{c} \tau} d \tau B_{c} \\
C_{d}=C_{c}, \quad D_{d}=D_{c}
\end{gathered}
$$

Detail derivations of these relations are well known and for instance, derived in the reference [1]. It is noted that $C_{c}$ and $D_{c}$ are not influenced by ZOH-sampling. For the rest of the thesis, $\mathrm{ZOH}$-sampling is assumed for discrete time sampling.

\subsection{Modal model in frequency-domain}

The Laplace transform

The Laplace transform ( $\mathrm{s}$-transform) converts linear time-variant differential equations to algebraic equations. The one-sided Laplace transform of a timedependent variable $q(t)$ is defined as:

$$
Q(s)=\mathcal{L}[q(t)]=\int_{0}^{\infty} e^{-s t} q(t) d t
$$

where $s \in \mathbb{C}$ is a square complex variable. It is assumed that $q(t)=0$ prior to $t=0$. It is said that function $q(t)$ in the time -domain is transformed into the function $Q(s)$ in the Laplace-domain. Form the practical view point, the Laplace variable is restricted to purely imaginary values $s=j \omega$ where $\omega[\mathrm{rad} / \mathrm{s}]$ can be any frequency of interest.

The modal model

Using the Laplace transform of Equation (2-1) and neglecting the initial conditions results in the frequency-domain equivalent given by 


$$
Z(s) Q(s)=F(s)
$$

with the dynamical stiffness $Z(s)=M s^{2}+C s+K$ and $s=j \omega$. Inverting Equation (2-21) yields:

$$
Q(s)=H(s) F(s)
$$

with $H(s)=Z(s)^{-1}$ the transfer function matrix. The transfer function matrix can be formulated in its modal form [2], [3] as:

$$
H(s)=\theta\left(s I_{n_{2}}-\Lambda\right)^{-1} L_{m}^{T}+\theta^{*}\left(s I_{n_{2}}-\Lambda^{*}\right)^{-1} L_{m}^{H}
$$

where superindex, ' $\mathrm{H}$ ' stands for Hermitian transpose. And the modal parameters $\lambda_{i}, \Theta_{i}, L_{i, m}^{T}$ are respectively the pole, mode shape and modal participation factor of mode $i$.

\subsection{Common -denominator models}

The common-denominator, also called scalar matrix fraction model [4], considers the relation between output $o$ and input $k$ as a rational fraction of two polynomials, of which the denominator polynomial is common for all input-output relations. The transfer function matrix $H(s)$ can be expressed as:

$$
H(s)=\frac{Z_{a d j}(s)}{|Z(s)|}
$$


with $Z_{\text {adj }}(s)$ the adjoin matrix, containing polynomials of order $2 n_{2}-1$. The common-denominator is given by the characteristic equation $|Z(s)|$, a polynomial in $s$ of order $2 n_{2}$, which roots are the poles of the structure. In general the commondenominator model can be expressed as follows

$$
H(s)=\frac{\left[\begin{array}{ccc}
B_{11}(s) & \ldots & B_{1 N_{k}}(s) \\
\vdots & \ddots & \vdots \\
B_{N_{o} 1}(s) & \ldots & B_{N_{o} N_{k}}(s)
\end{array}\right]}{A(s)}
$$

The relation between the modal model and the common-denominator model is obtained by considering the frequency response function between output $o$ and input $k$

$$
\begin{aligned}
H_{o k}(s) & =\sum_{i=1}^{n_{2}}\left(\frac{\theta_{o i} L_{k i}}{s-\lambda_{i}}+\frac{\theta_{o i}^{*} L_{k i}^{*}}{s-\lambda_{i}^{*}}\right) \\
& =\frac{B_{o k}(s)}{A(s)}
\end{aligned}
$$

From this equality it is clear that the structure poles are given by the roots of the denominator, $A(s)$ while the mode shapes and participation factors are obtained from singular value decomposition (SVD) of the residue matrix $Q_{i} \in \mathbb{R}^{l \times m}$ of mode $i$ :

$$
Q_{i}=\theta_{i} L_{i}^{T}
$$

with the elements of the residue matrix $Q_{i}$ given by:

$$
\begin{aligned}
Q_{o k, i} & =\lim _{s \rightarrow \lambda_{i}}\left(s-\lambda_{i}\right) H_{o k}(s) \\
& =\theta_{o i} L_{k i}
\end{aligned}
$$


with $\theta_{i}=\left[\begin{array}{llll}\theta_{1 i} & \theta_{2 i} & \ldots . . \theta_{l i}\end{array}\right]^{T}$ and $L_{i}=\left[\begin{array}{llll}L_{1 i} & L_{2 i} & \ldots . . L_{m i}\end{array}\right]^{T}$. It follows from modal analysis theory that this residue matrix is of rank 1 . But the common denominator model does not ensure rank 1 in the residue matrices of the measurements. Therefore, if there are poles with multiplicity 2 or more, then it is not possible to uniquely determine the corresponding mode shapes and modal participation vectors.

\subsection{State-space models in the frequency-domain}

Taking a Laplace transform of the Equation (2-6), the well known state-space model in frequency-domain is obtained:

$$
\left\{\begin{array}{c}
s Q(s) \\
s^{2} Q(s)
\end{array}\right\}=\left[\begin{array}{cc}
0 & I \\
-M^{-1} K & -M^{-1} C
\end{array}\right]\left\{\begin{array}{c}
q(s) \\
s q(s)
\end{array}\right\}+\left[\begin{array}{c}
0 \\
M^{-1} B_{2}
\end{array}\right]\{U(s)\}
$$

Reformulating Equation (2-29) results in the following

$$
\begin{gathered}
s X(s)=A_{c} X(s)+B_{c} U(s) \\
Y(s)=C_{c} X(s)+D_{c} U(s)
\end{gathered}
$$

where $X(s)=\left\{\begin{array}{c}Q(s) \\ s Q(s)\end{array}\right\} \in \mathrm{C}^{n}$ is the state vector. Identification algorithms based on a state-space model identify the system matrices $A_{c}, B_{c}, C_{c}$ and $D_{c}$ from the measurement data. The transfer function matrix between the outputs and inputs is then given by

$$
H(s)=C_{c}\left(s I_{n}-A_{c}\right)^{-1} B_{c}+D_{c}
$$

Similar as in time-domain, modal decomposition can be applied to this expression. 
By inserting the eigenvalue decomposition of $A_{c}$ (Equation 2-8) and definition of the participation factors $L_{c}^{T}$ and the observed mode shapes (Equation 2-17) in Equation 2-31, the following can be obtained

$$
H(s)=V_{c}\left(s I_{n}-\Lambda_{c}\right)^{-1} L_{c}^{T}+D_{c}
$$

Equation (2-32) is entirely equivalent to the more commonly used expression of the modal model in frequency given by Equation (2-23).

\subsection{Matrix fraction polynomial models}

The common-denominator model (scalar matrix fraction description) can be considered as a special case of multivariable transfer function models described using a Matrix Fraction Description (MFD), i.e. the ratio of two matrix polynomials. The multivariable transfer function models can be divided into two types of model, left MFD (LMFD) and a right MFD (RMFD). [5] [6].

\subsubsection{Left matrix fraction description (LMFD) models}

Left matrix fraction description (LMFD) models consider all input-output measurements simultaneously in the following form

$$
H(s)=A^{-1}(s) B(s)
$$

with $A(s)=I s^{n}+A_{n-1} s^{n-1}+\cdots \cdots+A_{0}$ a matrix polynomial with $l \times l$ matrix coefficients and $B(s)=B_{n} s^{n}+B_{n-1} s^{n-1}+\cdots \cdots+B_{0}$ a matrix polynomial with $l \times m$ matrix coefficients. This LMFD model can be obtained from the state-space 
model. The denominator coefficients are obtained by solving the following linear set of equations [7]:

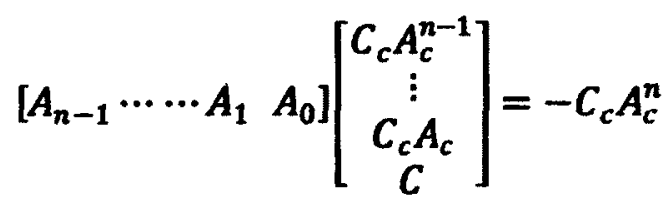

The numerator coefficients are obtained by considering the following set of equations:

$$
\begin{aligned}
{\left[\begin{array}{c}
H(s) \\
s H(s) \\
\vdots \\
s^{n} H(s)
\end{array}\right]=} & {\left[\begin{array}{c}
C_{c} \\
C_{c} A_{c} \\
\vdots \\
C_{c} A_{c}^{n}
\end{array}\right] X(s) } \\
& +\left[\begin{array}{cccc}
D_{c} & 0 & \ldots & 0 \\
C_{c} B_{c} & D_{c} & \ldots & 0 \\
\vdots & \ddots & \cdots & \vdots \\
C_{c} A_{c}^{n-1} B_{c} & C_{c} A_{c}^{n-2} B_{c} & \ldots & D_{c}
\end{array}\right]\left[\begin{array}{c}
I \\
s I \\
\vdots \\
s^{n} I
\end{array}\right]
\end{aligned}
$$

or in compact form as follows:

$$
H_{n}=O_{n} X(s)+\Gamma I_{n}
$$

Pre-multiplying Equation (2-36) by $\left[A_{0} \cdots A_{n-1} \eta\right]$ and using Equation (2-34) yields

$$
\left[\begin{array}{lll}
A_{0} & \cdots & A_{n-1}
\end{array}\right] \mathrm{H}_{\mathrm{n}}=\left[\begin{array}{llll}
A_{0} & \cdots & A_{n-1} & I
\end{array}\right] \mathrm{I}_{\mathrm{n}}
$$

The left-hand side is the same as $A(s) H(s)$ and thus by comparing this equation with Equation (2-33) the right hand side must be equal to $B(s)$. Knowing the coefficients of $A_{n}$, a generalized eigenvalue problem is obtained from $A(s)=0$ which results in $n \times l$ eigenvalues and the corresponding left eigenvectors [8]. The participation factors can be obtained from the coefficients of $B$ [5], or from a least 
squares problem in a second step estimation procedure known as Least Square Frequency Domain (LSFD) [3] method. Both the poles and participation factors are known, the frequency response functions (FRF) are a linear function of mode shapes.

\subsubsection{Right matrix fraction description (RMFD) models}

In case of the left matrix fraction description (LMFD) models, the size of the coefficient matrices is proportional to $l$ whereas for the right matrix fraction description (RMFD), it is proportional to $m$. In typical modal analysis application where the number of outputs is much higher than the number of inputs, this means that a huge number of mathematical poles are estimated by LMFD models. This drives the motivation for describing the FRF as RMFD models. The RMFD model is given by

$$
H(s)=B(s) A^{-1}(s)
$$

where $A(s)=I s^{n}+A_{n-1} s^{n-1}+\cdots \cdots+A_{0}$ a matrix polynomial with $m \times m$ matrix coefficients and $B(s)=B_{n} s^{n}+B_{n-1} s^{n-1}+\cdots \cdots+B_{0}$ a matrix polynomial with $l \times m$ matrix coefficients. This RMFD model can be considered as a LMFD model of the transposed transfer matrix $H^{T}(s)$.

$$
H^{T}(s)=A^{-T}(s) B^{T}(s)
$$

and by consequence similar relations between the state-space model and the RMFD model can be derived. In practice, since the number of input $m$ is typically much smaller than the number of modes $n_{2}$, RMFD models have a higher model order $n$ than LMFD models, but the denominator coefficients have smaller dimensions. 
Similar as for the LMFD model, the poles $\lambda_{t}$ and modal participation vectors are obtained from reformulating $A(s)=0$ into a generalized eigenvalue problem, resulting in $n \times m$ eigenvalues and the corresponding left eigenvectors. The mode shapes can be obtained from the $B$ coefficients or from a second step estimation procedure by solving a linear least-squares problem, since the modal model is linear in the mode shapes [9].

\subsection{Discrete-time frequency-domain models}

For time-domain models, a distinction is made between continuous-time and discrete-time models. Unlike in the time domain, system identification in frequencydomain does not require discrete-time models. It is, for instance, possible to identify a continuous-time frequency-domain model from samples of the Fourier transforms of the discrete time signals. However, these models using a Laplace-domain formulation, typically involve the inversion of a matrix containing powers of the frequency-axis of the data and thus one quickly runs into numerical conditioning problems and severe constraints to both the frequency range and the modal order range of the analysis. Previously, to solve this numerical problems, a number of orthogonal polynomial basis for the frequency-domain model have been proposed at the cost of increased computational time and memory requirements. This is the main rationale behind the formulation of discrete-time system identification models in frequency-domain. And at the same time, it is noted that an identified time- 
domain model can be analytically converted to frequency domain, which then can be used to compare with the non-parametric estimate of the frequency data by applying FFT to the sampled data.

The z-transform

The $z$-transform is the discrete-time analogy of the $s$-transform. It is defined as:

$$
X(z)=Z\left[x_{k}\right]=\sum_{k=0}^{\infty} x_{k} z^{-k}
$$

where $z \in \mathbb{C}$ is a scalar complex variable. By taking the $s$-transform of a continuoustime signal that equals a given discrete-time signal at the discrete samples and is zero elsewhere, it can be shown that the $z$-transform coincides with the $s$-transform by setting $z=e^{s \Delta t}[1]$. Therefore, a restriction to purely imaginary values of the $s$ variable in continuous-time corresponds to a restriction to values on the unit circle in discrete-time:

$$
z=e^{j \omega \Delta t}
$$

So, any power of $z$ variable does not increase the range of values as it boils down to a rotation in complex z-plane. As a result, the inversion of a matrix containing the frequency-axis of the data in $z$-domain is not affected by numerical conditioning problem.

The proposed continuous time models i.e. the modal model, the commondenominator model, the LMFD and RMFD models and state-space models all have their discrete-time equivalent, which are of the same form but the basis functions $s^{n}$ are replaced by $z^{n}$. 


\subsection{Primary identification data}

In the previous sections different types of mathematical models both in time and frequency domain are briefly discussed. In this section the primary data which are used to identify mathematical models to describe the behaviour of a system or structure are discussed. First, the basis of identifying mathematical models for the case of input-output measurements is introduced and then the idea is extended to output only measurements. Next, the difference between data driven identification models and average-based spectral (ABS) function driven identification models with reference to characteristics and requirements of sample data are discussed.

\subsubsection{Input-output measurements}

It is well known that linear time invariant systems can be modeled by using from input-output measurements. The identified parametric model essentially contains the same information of the studied system as the original non-parametric data, but is often preferred because of its compact form and possible physical interpretation. Previous studies on the subject of system identification include [10], [11] and [12] in the time- domain, and in the frequency-domain [13]. Identification of modal model for linear time invariant mechanical systems or structures from input-output data is known as Experimental Modal Analysis (EMA). Experimental modal analysis starts from measuring impulse responses when artificially excited in one or more input locations. The impulse responses are the outputs of the system when excited by an impulse applied at any of the $m$ input locations. These $m$ response vectors are 
usually combined in a $l \times m$ impulse response matrix. Under zero initial conditions, it is straightforward to prove from Equation $(2-18)$ that the impulse response matrices $h(k) \in \mathbb{R}^{l \times m}$ can be computed from the system matrices as follows

$$
\begin{aligned}
& h(0)=D_{d} \\
& h(k)=C_{d} A^{k-1} B_{d}
\end{aligned}
$$

The relation between impulse responses and state-space matrices (Equation 2-42) originates from the famous paper by Ho and Kalman [14]. Many identification methods (the so-called realization methods) are based on this property. In the final step, the impulse response can be decomposed to modal model from which the system modal properties are identified.

\subsubsection{Output-only measurements: stochastic approach}

In some applications, it is very difficult to apply artificial force to the system and thus one has to rely upon available ambient sources as external excitation. It is practically impossible to measure this natural excitation and consequently outputs are the only information that can be passed to the system identification algorithms. This type of system identification technique is known as Operational Modal Analysis (OMA). Operational modal analysis is particularly suitable in civil engineering where it is very difficult and expensive to excite large structures e.g. bridges and buildings with shaker or hammer to obtain artificially induced vibration levels that exceed the natural vibrations due to traffic or wind. In OMA application, the role of the input signal is replaced by the assumption that the response is a realization of a stochastic process with unknown white noise as input. Identifying system parameters from 
these responses only is referred to as stochastic system identification [15]. It has been shown that [11] for zero mean stationary stochastic process; correlations between the output responses play the same role as impulse response in the inputoutput systems. So just like impulse response, correlations of the output can be factorized into system matrices from which system modal properties can be easily obtained. In frequency-domain, the relation between input and output response can be described in terms of power spectra as follows

$$
S_{y u}=H(\omega) S_{u u}
$$

where $S_{y u}, H(\omega)$ and $S_{u u}$ are the power spectra between input and output, the frequency spectra and auto spectra between inputs. The power spectrum of a white noise input is

$$
S_{u u}=R_{u u}=\sigma_{u}^{2} I
$$

here $R_{u u}$ is the spectrum of white input noise. So from Equation (2-43),

$$
S_{y u}=H(\omega) \sigma_{u}^{2}
$$

i.e. the cross power spectrum is proportional to the frequency response of the system. Equation (2-45) shows that frequency spectra of the system can be directly obtained from the cross-power spectrum under the assumption of white noise input.

In the field of stochastic system identification one can generally divide the identification techniques in two basic subcategories of data-driven stochastic identification algorithms and correlation or power spectral density driven stochastic identification algorithms. 
Data-driven stochastic identification algorithms

In data-driven stochastic identification algorithms, the algorithms start from the output time sequences or output spectra. The famous identification algorithm, Auto Regressive Moving Average (ARMA), falls within this category. An ARMA [12] model is written as:

$$
y_{k}+\alpha_{1} y_{k-1}+\ldots+\alpha_{n_{\alpha}} y_{k-n_{\alpha}}=e_{k}+\gamma_{1} e_{k-1}+\ldots+\gamma_{n_{y}} e_{k-n_{\gamma}}
$$

where, as before, $y_{k}$ is the output vector and $e_{k}$ a white noise vector sequence. The left-hand side of Equation (2-46) is called the Auto-Regressive (AR) part and the right-hand side the Moving Average (MA) part, hence the name of the model. The matrices $\alpha_{i} \in \mathbb{R}^{l \times l}$ are the AR matrix parameters whereas matrices $\gamma_{i} \in \mathbb{R}^{l \times l}$ are the MA matrix parameters. It has been shown that [15] ARMA model that is deduced from a state-space model has the same AR order $n_{\alpha}$ as MA order $n_{r}$ A major drawback of the formulation given by Equation (2-46) is that for the coefficients $\gamma_{i} \neq 0$ the identification problem becomes highly non-linear in the system parameters. For systems with large number of outputs $l$, which is typically the case of modal testing, the algorithms become computationally intensive and thus inefficient in practical applications.

A second type of stochastic data driven models are stochastic state-space models. State-space models are derived from the output measurement response. Prominent references on stochastic state-space model identification by subspace methods include the study by [11] and their application to civil engineering structures [16]. The stochastic time-domain state-space model is given by 


$$
\begin{aligned}
x_{k+1} & =A x_{k}+w_{k} \\
y_{k} & =C x_{k}+v_{k}
\end{aligned}
$$

with $w_{k}$ and $v_{k}$, the white noise representing the immeasurable ambient force. In [9], a frequency-domain counterpart subspace algorithm is developed to identify state-space models from output Fourier spectra only.

Correlation or power spectral density driven stochastic identification

These algorithms first estimate the power spectra between the outputs and certain reference sensors and then from these functions a deterministic model related to stochastic model is extracted. In this section the modal decomposition of power densities and correlations is briefly discussed. It has been shown that the modal decomposition of an FRF matrix $H(\omega) \in \mathbb{C}^{l \times m}$ can be written as follows [3]:

$$
H(\omega)=\sum_{k=1}^{n_{2}} \frac{\left\{v_{k}\right\} \times<1_{k}^{T}>}{j \omega-\lambda_{k}}+\frac{\left\{v_{k}^{*}\right\} \times<l_{k}^{H}>}{j \omega-\lambda_{k}^{*}}
$$

where $n_{2}$ is the number of modes. $\left\{v_{k}\right\}$ is an $l \times 1$ vector of the $k^{\text {th }}$ mode shape, $\left\langle l_{k}^{T}\right\rangle$ is the $1 \times m$ vector of the $k^{\text {th }}$ modal participation factor and $\lambda_{k}$ is the $k^{\text {th }}$ system pole, which are related to the modal frequencies and damping ratios. The FRF relates the input spectra $S_{\mathrm{uu}} \in \mathbb{C}^{m \times m}$ and output spectra $S_{\mathrm{yy}} \in \mathbb{C}^{l \times l}$ as follows [12]:

$$
S_{y y}(\omega)=H(\omega) S_{u u} H(\omega)^{H}
$$

Since white noise has a constant power spectrum, it is independent of frequency [17]. The modal decomposition can now be reformulated as follows [18]

$$
\mathrm{S}_{\mathrm{yy}}(\omega)=\sum_{\mathrm{k}=1}^{\mathrm{n}_{2}} \frac{\left\{\mathrm{v}_{\mathrm{k}}\right\} \times\left\langle\mathrm{g}_{\mathrm{k}}^{T}\right\rangle}{j \omega-\lambda_{\mathrm{k}}}+\frac{\left\{\mathrm{v}_{\mathrm{k}}^{*}\right\} \times\left\langle\mathrm{g}_{\mathrm{k}}^{H}\right\rangle}{j \omega-\lambda_{\mathrm{k}}^{*}}+\frac{\left\{\mathrm{g}_{\mathrm{k}}\right\} \times\left\langle\mathrm{v}_{\mathrm{k}}^{T}\right\rangle}{-j \omega-\lambda_{\mathrm{k}}}+\frac{\left\{\mathrm{g}_{\mathrm{k}}^{*}\right\} \times\left\langle\mathrm{v}_{\mathrm{k}}^{H}\right\rangle}{-j \omega-\lambda_{\mathrm{k}}^{*}}
$$


where $\left\langle\mathbf{g}_{\mathbf{k}}^{\mathbf{T}}\right\rangle$ are operational reference factors, which replace the modal participation factors in the input-output case of experimental modal analysis. It should be noticed that the modal decomposition of the power densities of the outputs has a symmetry in the poles i.e. both the positive and negative poles are present in the model. This symmetry is referred to as 4-quadrant symmetry. Because of the similarity between the modal decomposition of the auto and cross spectral densities of the outputs and the modal decomposition of the FRFs, modal parameter estimation techniques for FRFs can be applied to the power spectra in the output-only case. Taking the inverse Fourier transform (IFT) of Equation (250), yields the correlation function matrix at positive and negative lag time $k$ [19]. The time-domain modal decomposition of the causal part of the correlations is similar to the modal decomposition of impulse response functions (IRF) and hence classical modal parameter estimators can be used.

\subsection{Conclusions}

This chapter presents several models of vibrating structures. They differ whether they are in continuous-time or discrete-time; whether they are input-output or output-only models and whether they are in time domain or in frequency domain. A distinction is also made based on the type of the data used for identification i.e. data driven or spectral/correlation driven. These models form the basis of the 
identification algorithms presented in the next chapter. It will be shown how these models can be obtained from measurements. 


\section{Chapter 3 - Stochastic System}

\section{Identification}

\subsection{Introduction}

In previous chapter, several equivalent models for a vibrating structure are presented. In this chapter an overview on the system identification methods utilized to obtain estimates of the parameters of the dynamic models discussed in the previous chapter is presented. Even though the main focus of the thesis is operational modal analysis, a brief overview of Experimental Modal Analysis (EMA) algorithms is also presented in order to comprehend the underlying concept of Operational Modal Analysis (OMA) applications. Next the concepts of EMA are extended to OMA cases with the assumption of white noise as input excitation and different stochastic identification algorithms are discussed. These algorithms are divided into the categories of time, frequency and spatial-domain algorithms. This chapter ends with the associated nonparametric processing of primary data required by the identification algorithms. 


\subsection{A literature review of EMA algorithms}

The traditional methods of system identification include a different class of methods based on different general approached and assumptions [2]. These traditional methods from the early 1980 's are developed to address the problem of modal identification of structural and mechanical systems with low damping. In early studies, the systems are assumed to operate under noise free environments. The influences of noise and uncertainty on the accuracy of system identification algorithms are ignored. Later on, noise was also incorporated. A few extensively used methods are reviewed here. Complex exponential (CE) algorithm based on Prony's method [2] is the first important single-input single-output (SISO) parametric modal identification method. Prony's method extracts periodic information from a uniformly sampled signal by building a series of damped complex exponentials, or sinusoids. This allows the estimation of frequency, amplitude, phase, and damping components of a signal. Although this method is normally applied to data of free decay response of a system generated by release of an initial condition, it is also applied quite generally to analyze impulse response function data. Since impulse response function data is scaled to include the forcing condition, use of this method on impulse response function data yields properly scaled modal parameters that can be used to calculate generalized mass and stiffness. This is not possible if free decay responses are used. By extending the concept of Prony's method, Ibrahim time domain (ITD) method [20] was the first 
modal identification method formulated for single input multiple output (SIMO) systems. In the SIMO approach, the method computes the poles and residues in one step based on a specific initial vibration condition of the structure. A number of different initial conditions can be considered to establish the required system behaviour information analogous to the practice of using several exciter positions in ordinary single input modal surveys until all important modes are excited. Although this technique is based upon free decay data, the ITD method can also be used with operating inputs if the free decay is computed from the operating inputs by using random decrement averaging or from measured auto and cross correlation functions. Again, it should be emphasized that this can only be done if there are no poles or zeros in the input spectrum in the frequency range of interest. The first multiple-input multiple-output modal identification method, Polyreference Least Square Complex Exponential (p-LSCE) [21], was developed as an extension of the least squares complex exponential algorithm. In contrast to the ITD approach, the Poly-Reference Time Domain approach utilizes all measured damped complex exponential information, from all references or initial conditions simultaneously in the estimation of modal frequencies. Since the cases of multiple initial conditions or reference data are considered in the algorithm, the p-LSCE method is the first algorithm able to handle the vibration behaviour of systems with closely-spaced or repeated roots. It overcomes the problem that sometimes occurs when using a SIMO method where one mode of vibration may not be excited because the excitation may be located close to a node of the structure. The Eigensystem realization algorithm 
(ERA) [22] was developed based on the general state-space description for linear dynamic systems. This method is similar to the other damped complex exponential methods in that all involve solutions of a matrix eigenvalue problem. Since the ERA approach utilizes multiple reference data, the ERA approach is similar to the p-LSCE approach. This means that repeated roots can be identified with this approach as in the case of p-LSCE approach. Other significant attributes of this approach include the extensive use of accuracy indicators to assess effects of noise and nonlinearities as well as rank information provided by singular value decomposition techniques. A well-known unified matrix polynomial approach that includes many early system identification algorithms for modal identification is the common algorithm framework based on multiple-dimension auto-regression exogenous (ARX) model [23].

In frequency-domain, one of the earliest system identification methods is the frequency response function (FRF) peak picking method [24]. However, the FRF peak picking technique is often found to be inaccurate, especially for mode shape and damping estimation. A more advanced method, Complex Mode Indicator Function (CMIF) alleviates some of these difficulties by applying Eigenvalue Decomposition or Singular Value Decomposition (SVD) of FRF matrix while retaining the advantages of the classical pick picking method [25]. In practical calculation, the normal matrix formed from the FRF matrix, is calculated at each spectral line; then eigenvalues of this matrix are obtained. The CMIF plot is a plot of these eigenvalues as a function of frequency in log magnitude scale. Each peak of 
CMIF plot represents a single degree of freedom system and the corresponding frequency is identified as damped natural frequency. A method to estimate damping using the enhanced frequency response function was proposed subsequently [25]. There are a number of other frequency-domain methods in the literature, and a complete review of these methods can be found in the reference in [2].

In the next subsection, the popular EMA algorithms ERA method is briefly presented as later its performance under the assumption of white input noise is compared to other OMA algorithms.

\subsubsection{Eigensystem realization algorithm}

The ERA approach is based on well-established realization (state-space) theory using the concepts of controllability and observability. The approach determines a complete discrete time state-space model based upon the important principles of minimal realization theory by Ho and Kalman [14]. The ERA approach begins with a block data matrix formulated from damped complex exponential functions, such as free decay responses or pulse response. The response of the structure in discrete time domain to a unit pulse is called pulse response, or markov parameters [1]. The markov parameters contain system information, and are function system matrices as shown in Section 2.10.1. The ERA method uses the pulse response $h(k)$ to form a matrix known as the Hankel matrix: 


$$
H(k-1)=\left[\begin{array}{cccc}
h(k) & h(k+1) & \vdots & h(k+c) \\
h(k+1) & h(k+2) & \vdots & h(k+c+1) \\
\cdots & \cdots & \vdots & \cdots \\
h(k+r) & h(k+r+1) & \vdots & h(k+c+r)
\end{array}\right]
$$

Substituting $k=1$ in Equation (3-1) and replacing the markov parameters (Equation 2-42) yields,

$$
H(0)=\left[\begin{array}{cccc}
C_{d} B_{d} & C_{d} A_{d} B_{d} & \vdots & C_{d} A_{d}^{c-1} B_{d} \\
C_{d} A_{d} B_{d} & C_{d} A_{d}^{2} B_{d} & \vdots & C_{d} A_{d}^{c} B_{d} \\
\cdots & \cdots & \vdots & \cdots \\
C_{d} A_{d}^{r-1} B_{d} & C_{d} A_{d}^{r} B_{d} & \vdots & C_{d} A_{d}^{r+c-2} B_{d}
\end{array}\right]=P_{o} Q_{c}
$$

where $P_{o}$ and $Q_{c}$ are given by:

$$
\begin{aligned}
& P_{o}=\left[\begin{array}{llllll}
C_{d} & C_{d} A_{d} & C_{d} A_{d}^{2} & \cdots & \cdots & C_{d} A_{d}^{r-1}
\end{array}\right]^{T} \\
& Q_{c}=\left[\begin{array}{llllll}
B_{d} & A_{d} B_{d} & A_{d}^{2} B_{d} & \cdots & \cdots & A_{d}^{c-1} B_{d}
\end{array}\right]
\end{aligned}
$$

Performing singular value decomposition of $H(0)$ yields:

$$
H(0)=\mathrm{R} \Sigma \mathrm{S}^{\mathrm{T}}
$$

where $\Sigma$ is a diagonal matrix with the singular values in the diagonal, and the matrices $R$ and $S$ are square and unitary. The matrices $R_{N}, \Sigma_{N}$ and $S_{N}$ are obtained eliminating the rows and columns corresponding to small singular values produced by computational modes. Since $\Sigma_{N}$ is a diagonal matrix Eq. (3-4) can be expressed as,

$$
H(0)=\left(\mathrm{R}_{\mathrm{N}} \Sigma_{\mathrm{N}}^{\frac{1}{2}}\right)\left(\Sigma_{\mathrm{N}}^{\frac{1}{2}} S_{\mathrm{N}}\right)
$$

From Eq. (3-2) it can be shown that, 


$$
\begin{aligned}
& P_{o}=R_{N} \Sigma_{N}^{\frac{1}{2}} \\
& Q_{c}=\Sigma_{N}^{\frac{1}{2}} S_{N}^{T}
\end{aligned}
$$

Using the discrete-time state space form of the equation and the Markov parameters, Equation (3-1) becomes:

$$
\begin{aligned}
& H(k-1)=P_{o} A_{d}^{k-1} Q_{c} \\
& =\left[\begin{array}{c}
C_{d} \\
C_{d} A_{d} \\
\vdots \\
C_{d} A_{d}^{r-1}
\end{array}\right] A_{d}^{k-1}\left[A_{d} x(0) \quad A_{d}^{2} x(0) \quad A_{d}^{3} x(0) \quad \ldots \quad \ldots \quad A_{d}^{c-1} x(0)\right]
\end{aligned}
$$

For $k=2$

$$
H(1)=P_{o} A_{d} Q_{c}
$$

Combining Equations (3-6), (3-7), (3-8) the system and the output matrices can be found as:

$$
\begin{aligned}
H(1) & =\mathrm{R}_{\mathrm{N}} \Sigma_{N}^{1 / 2} A_{d} \Sigma_{\mathrm{N}}^{1 / 2} \mathrm{~S}_{\mathrm{N}}^{\mathrm{T}} \\
A_{d} & =\Sigma_{\mathrm{N}}^{-1 / 2} \mathrm{R}_{\mathrm{N}}^{\mathrm{T}} H(1) \mathrm{S}_{\mathrm{N}} \Sigma_{\mathrm{N}}^{-1 / 2} \\
C_{d} & =\left[\begin{array}{ll}
I & 0
\end{array}\right] \mathrm{R}_{\mathrm{N}} \Sigma_{\mathrm{N}}^{1 / 2}
\end{aligned}
$$

Where $l$ is an identity matrix and 0 is a zero matrix, both of proper dimensions for the necessary matrix products. The natural frequencies and the mode shapes are obtained from $A_{d}$ and $C_{d}$ matrices. A rigorous mathematical proof of this is presented in [1]. The natural frequencies $\omega_{i}$ and $\xi_{i}$ are obtained from the eigenvalues $\Lambda_{d}$ of the state matrix $A_{d}[26]$ :

$$
\left[\begin{array}{lll}
\ddots & & \\
& \omega_{i} & \\
& & \ddots
\end{array}\right]=[\Omega]=\frac{\left|\Lambda_{c}\right|}{2 \pi}
$$




$$
\left[\begin{array}{lll}
\ddots & & \\
& \xi_{i} & \\
& & \ddots
\end{array}\right]=\frac{-100 \times \text { real }\left|\Lambda_{c}\right|}{|\Omega|}
$$

where $\Lambda_{c}=\ln \Lambda_{d} / \Delta t, \Lambda_{c}$ is the eigenvalue of continuous time state space matrix $A_{c}$ and $\Delta t$ is the sampling time. Accuracy indicators such as the rank of the block data matrix, modal amplitude coherence, modal phase collinearity, and data reconstruction are used to identify the final set of modal parameters. The Eigensystem Realization Algorithm (ERA) generates a matrix polynomial characteristic equation with matrix dimension equal to the number of response sensors $N_{o}$ and low model order. The primary limitation of this and other low order methods is the amount of computer memory required to solve the problem for cases with a large number of response sensors.

\subsection{OMA algorithms}

In general, most OMA algorithms are essentially extensions of the traditional EMA algorithms. In this section the most common and most widely used OMA algorithms are discussed.

\subsubsection{Time-domain algorithms}

The utilization of Auto-Regressive Moving Average (ARMA) procedure for estimating modal parameters using response data only has been developed in the 1970's [27], [28], [29] to estimate the characteristics of the structural dynamic behaviour of buildings excited by wind forces by assuming the input forces as white 
random noise. However, it is since early 1990's that researchers have more seriously considered utilizing output response only based techniques.

\section{Natural Excitation Technique (NExT) and Other Similar Algorithms}

The Natural Excitation Technique (NExT) [30], one of the earliest OMA algorithms was developed during the early 1990 's for modal testing of the vertical-axis wind turbines. The theoretical basis of NExT is the cross-correlation functions between the response measurements and a single reference measurement satisfy the homogeneous differential equation of motion of the linear system and, therefore, can be treated as free responses. The Natural Excitation Technique (NExT) has been used extensively for numerical and experimental analysis showing that, even in the case of band-limited ambient vibrations with questionable stationarity, free responses can be obtained reliably with sufficient frequency content to detect the dominant modes of vibration.

Rewriting the equations of motion for $n_{2}$ degree of freedom, time invariant, linear system described by Equation (2-1)

$$
M \ddot{q}(t)+C \dot{q}(t)+K q(t)=f(t)
$$

Post multiplying this equation by the displacement of any of the degrees of freedom measured $q_{i}\left(t_{1}\right)$, referred to as the reference signal, and taking the expected value of each side yields:

$$
M R_{\tilde{q} q_{i}}\left(t, t_{1}\right)+C R_{\dot{q} q_{i}}\left(t, t_{1}\right)+K R_{q q_{i}}\left(t, t_{1}\right)=R_{F q_{i-}}\left(t, t_{1}\right)
$$

where $R($.$) denotes the vector of correlation functions and t_{1}$ denotes different time time instant. For weakly stationary processes, it can be shown that [24]: 


$$
R_{q q_{i}}(\tau)=R_{\dot{q} q_{i}}(\tau)=-R_{q q_{i}}(\tau)
$$

where $(\tau)=t-t_{1}$. Assuming that the displacement, velocity and acceleration processes are weakly stationary and uncorrelated with future disturbances and taking the fourth derivative of the Equation (3-14):

$$
M R_{\tilde{q} \tilde{q}_{i}}+C R_{\tilde{q} \tilde{q}_{i}}+K R_{\tilde{q} \tilde{q}_{i}}=0
$$

Equation (3-16) shows that the cross-correlation function of the responses of the structure with a reference signal satisfies the homogeneous equation of motion and can be treated as free responses. So, under the assumption that the system is excited by stationary white noise, correlation functions between the response signals can also be expressed as a sum of decaying sinusoids. Each decaying sinusoid has a damped natural frequency and damping ratio that is identical to that of a corresponding structural mode. Consequently, the classical modal parameter techniques using impulse response functions as input like p-LSCE, ERA and ITD methods are also appropriate to extract the modal parameters from the response only data measured under operational conditions.

\section{Prediction Error Method (PEM) and Instrumental Vartable (IV) Method}

A Prediction-Error Method (PEM) based on the ARMA model discussed in Chapter 2 can be derived to identify modal parameters by minimizing the prediction error [12]. This approach results in a highly nonlinear optimization problem and algorithms based on this approach is sensitive to initial values and is computationally intensive with convergence not guaranteed; all of which makes it 
unsuitable for OMA purposes, especially for analyzing large structures. A MIMO version of this algorithm, PEM-ARMAV (Vector ARMA), was proposed by Andersen [31]. The nonlinear nature of the ARMA based PEM arises due to the Moving Average (MA) polynomial coefficients. For the purpose of identifying modal parameters though, only the Auto-Regressive (AR) polynomial coefficients are needed. The Instrument Variable (IV) method [32] uses this approach for system parameter identification, thus avoiding the limitations of PEM. The ARMA representation of a vibrating structure is given by Equation (2-46):

$$
y_{k}+\alpha_{1} y_{k-1}+\ldots+\alpha_{n_{\alpha}} y_{k-p}=e_{k}+\gamma_{1} e_{k-1}+\ldots+\gamma_{n_{r}} e_{k-p}
$$

If the ARMA order $p$ times the number of outputs $l$ is equal to or larger than the system order $n(p l \geq n)$, the system poles are included in the model. The idea of the system identification is to "fit" such a model to measured data $y_{k}$. A good parameter estimation method should extract the maximum information from the data, leaving residuals $e_{k}$ that are uncorrelated with past data. This is formally written as:

$$
\forall i>0: \mathrm{E}\left[e_{k} y_{k-i}^{T}\right]=\mathrm{E}\left[e_{k}\right]\left[y_{k-i}^{T}\right]=0
$$

where $\mathrm{E}$ is the expectation operator. The first equality says that $e_{k}$ and $y_{k-i}$ are uncorrelated; and the second equality follows from the zero-mean property of the noise sequence. If on the other hand, the residuals are correlated with past data, they still contain useful but unmodeled information and the model is not ideal. The derivation of the IV method starts by imposing conditions like (3-17) to the ARMA model in order to eliminate the right hand side of Equation (2-46). The "oldest" noise term in Equation (2-46) is $e_{k-p}$; so by post-multiplying the ARMA model with 
$y_{k-p-i}^{T}$ and by taking expected value, the following expression can be obtained

$$
\forall>0, \mathrm{E}\left[y_{k} y_{k-p-i}^{T}\right]+\alpha_{1} \mathrm{E}\left[y_{k-1} y_{k-p-i}^{T}\right]+\ldots+\alpha_{p} \mathrm{E}\left[y_{k-p} y_{k-p-t}^{T}\right]=0
$$

Because of stationary assumption, the correlation function $R_{i}$ at lag time $i$ can be defined as follows

$$
\mathrm{E}\left[y_{k} y_{k-i}^{T}\right]=\mathrm{E}\left[y_{k+i} y_{k}^{T}\right]=R_{i}
$$

Thus the basic IV equation can be written in terms of the output covariances:

$$
\forall>0, R_{p+i}+\alpha_{1} R_{p+i-1}+\ldots+\alpha_{p} R_{i}=0
$$

By writing down the equation for all available time lags $i$, the AR parameters can be estimated by solving the resulting over-determined set of equations in a least squares sense. Finally, the eigenvalues and the observed mode shapes are obtained from the eigenvalue decomposition of the companion matrix of the AR coefficients. A more general discussion on the formulation of the IV methods can be found in [12].

\section{Covariance-driven Stochastic Realization-based algorithms (SSI-COV)}

Covariance driven stochastic realization based algorithms are inspired from the classical realization theory as explained by Ho and Kalman [14]. The deterministic system realization method is subsequently refined by use of Singular Value Decomposition (SVD) to reduce the effect of noise [33] \& [34]. The ERA method belongs to this class of algorithm commonly used in EMA.

The discrete-time deterministic state-space model is given by Equation (2-18)

$$
\begin{aligned}
& x_{k+1}=A_{d} x_{k}+B_{d} u_{k} \\
& y_{k}=C_{d} x_{k}+D_{d} u_{k}
\end{aligned}
$$


This discrete-time state-space model is a deterministic formulation, requiring complete knowledge of the input values. For an output-only system the sampled excitation vector $u_{k}$ is not measured due to practical reasons. Consequently, the model formulation is adapted to a stochastic model. To accomplish this, input terms $B u_{k}$ and $D u_{k}$ in Equation (2-18) are replaced with stochastic white noise terms. The discrete-time state-space model can now be written as follows:

$$
\begin{aligned}
x_{k+1} & =A_{d} x_{k}+w_{k} \\
y_{k} & =C_{d} x_{k}+v_{k}
\end{aligned}
$$

where $w_{k}$ and $v_{k}$ are white, zero-mean stochastic processes, independent of the state vector. Assuming stationarity of the state vector and whiteness of the input terms, it can be shown that the correlations of the outputs $R_{i}$ can be factorized into a triplet containing the state matrices [11]:

$$
R_{i}=C_{d} A_{d}^{i-1} G
$$

where $R_{i}$ is defined as $R_{i}=\mathrm{E}\left[\mathrm{y}_{\mathrm{k}+1} \mathrm{y}_{\mathrm{k}}^{\mathrm{T}}\right]$ with $\mathrm{E}$ denoting the expectation operator, and $\mathrm{G}=\mathrm{E}\left[\mathrm{x}_{\mathrm{k}+1} \mathrm{y}_{\mathrm{k}}^{\mathrm{T}}\right]$ as next-state output covariance matrix. The basis of SSI can be derived from Equation (3-22) which demonstrates that decomposition of the correlations yields the state-space matrices, which contain the structural parameters of the system. The correlations can be estimated from the data as:

$$
R_{i}=\frac{1}{N} \sum_{k=0}^{N-1} y_{k+i} y_{k}^{r}
$$

where $y_{k}$ is a column vector of measured response at the instant $k \Delta t$ and $N$ is the number of samples. These correlations significantly compress the data while 
maintaining the crucial modal information. The estimation of correlation from data is discussed in detail in the next section.

In operational modal analysis, data from multiple sensors are used to obtain accurate mode shapes. As all the sensors contain essentially the same information with respect to frequency and damping ratios, there is often redundancy in the data. Due to this, the computational requirements can be greatly reduced by calculating the data correlations with respect to a small set of reference sensors. A block Toeplitz matrix of data correlations at different time lags is then assembled as follows:

$$
T_{1 \mid i}^{r e f}=\left[\begin{array}{cccc}
R_{i}^{r e f} & R_{i-1}^{r e f} & \cdots & R_{1}^{r e f} \\
R_{i+1}^{r e f} & R_{i}^{r e f} & \cdots & R_{2}^{r e f} \\
R_{2 i-1}^{r e f} & R_{2 i-2}^{r e f} & \cdots & R_{i}^{r e f}
\end{array}\right]
$$

where $R_{i}^{r e f}=\mathrm{E}\left[y_{k+i}\left(y_{k}^{r e f}\right)^{T}\right], y_{k}^{r e f}$ is an $r \times 1$ vector of the sampled responses for the reference sensors at the time instant $k, r$ is the number of reference sensors, and $i$ is the parameter which defines the number of time lags of the correlations to be used. This parameter must satisfy the relation $r \times i>n$, where $n$ is the order of the statespace model. Based on Equation (3-22), the block Toeplitz matrix can be expressed as follows:

$$
T_{1 \mid i}^{r e f}=\left[\begin{array}{c}
C_{d} \\
C_{d} A_{d} \\
C_{d} A_{d}^{i-1}
\end{array}\right]\left[\begin{array}{lll}
A_{d}^{i-1} G^{r e f} & \cdots A_{d} G^{r e f} \quad G^{r e f}
\end{array}\right]=\mathrm{O}_{\mathrm{i}} \Gamma_{\mathrm{i}}^{\text {ref }}
$$


where $O_{1}$, the extended observability matrix, $\Gamma_{i}^{\text {ref }}$ is the reversed extended stochastic controllability matrix, both of which are rank of $n$. These factors can be estimated by Singular Value Decomposition (SVD) of the block Toeplitz matrix as follows:

$$
T_{1 k}^{r e f}=U S V^{T}=\left[\begin{array}{ll}
U_{1} & U_{2}
\end{array}\right]\left[\begin{array}{cc}
S_{1} & 0 \\
0 & 0
\end{array}\right]\left[\begin{array}{l}
V_{1}^{T} \\
V_{2}^{T}
\end{array}\right]=U_{1} S_{1} V_{1}^{T}
$$

where $S_{1}$ is a diagonal matrix containing singular values in descending order. Using Equations (3-25) and (3-26) the internally balanced realization may be obtained as follows:

$$
\begin{aligned}
& \mathrm{O}_{\mathrm{i}}=\mathrm{U}_{1} \mathrm{~s}_{1}^{1 / 2} \\
& \Gamma_{1}^{\mathrm{ref}}=\mathrm{S}_{1}^{1 / 2} \mathrm{~V}_{1}^{\mathrm{T}}
\end{aligned}
$$

As the true model order of the system is unknown, solutions of increasing model orders, from 1 to $n_{\max }$, where $n_{\max }$ is the maximum model order, are obtained by taking an increasing number of singular values from SVD to determine the estimates of $\mathrm{O}_{\mathrm{i}}$ and $\Gamma_{\mathrm{i}}^{\text {ref }}$. For each model order, the state matrices $A_{d}$ and $C_{d}$ can be extracted fromo $\mathrm{i}_{1}$ and $\Gamma_{1}^{\mathrm{ref}}$. The eigenvalue decomposition of $A_{d}$ yields the system's complex eigenvalues and vectors as follows:

$$
A_{d}=\Psi \Lambda_{d} \Psi^{-1}
$$

where $\Psi$ is the complex eigenvector matrix and $\Lambda_{d}$ is the diagonal matrix which contains the discrete-time eigenvalues $\mu_{i}$, which are directly related to the system poles $\lambda_{i}$. The poles contain the modal frequencies $\omega_{i}$ and damping ratios $\xi_{i}$ as follows: 


$$
\lambda_{i}=\frac{\log _{e}\left(\mu_{i}\right)}{\Delta t}=-\xi_{i} \omega_{i}+j \sqrt{1-\xi_{i}^{2}} \omega_{i}
$$

The modal frequencies are then obtained as follows:

$$
\omega_{i}=\frac{\operatorname{imag}\left(\lambda_{i}\right)}{2 \pi}
$$

and the damping ratios are calculated as follows:

$$
\xi_{i}=\frac{-\operatorname{real}\left(\lambda_{i}\right)}{\left|\lambda_{i}\right|}
$$

The mode shapes are obtained as follows:

$$
V_{c}=V_{d}=C_{c} \Psi=C_{d} \Psi
$$

where $C_{c}$ can be obtained as the first $l$ rows of $\mathrm{O}_{1}$ as seen in Equation (3-25)

In theory the order of the system can be determined by counting the number of nonzero singular values in the matrix $S$. However, when analyzing real structures there is noise in the data which results in the matrix $S$ having all non-zero singular values. This noise may be a result of:

- Modeling assumptions

- Measurement inaccuracies

- The use of finite data sets to compute estimates of the correlations

- Non-stationarity and non-linearity behaviour of the data

In order to overcome the uncertainty problem of an unknown system order, a stabilization diagram is constructed. For a stabilization diagram, a set of model orders are identified by including a different number of singular values and vectors

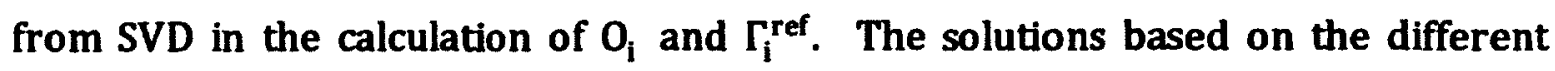


model orders are presented in the stabilization diagram, allowing for an experienced analyst to distinguish between true solutions and spurious solutions, which are a result of noise in the data. This technique is outlined herein. A more indepth discussion of the SSI method can be found in the reference [15]. Application of SSI-COV to actual bridge monitoring in the field can be found in [35].

\section{Data-driven Stochastic Reallzation-based algorithms (SSI-DATA)}

The SSI-DATA [36], [16] algorithm involves projecting the row space of the future outputs into row space of the past outputs by means of $Q R$ decomposition of the data Hankel matrix. This step is different from the SSI-COV method as, in this case, the data reduction is achieved by means of $Q R$ decomposition rather than the covariance calculation in the SSI-COV method. The QR decomposition is followed by the application of SVD to the computed projection matrix to obtain its Kalman filter state. Finally least square approach is used to extract the modal parameters. This algorithm is numerically more robust as it uses a square root algorithm, whereas in the SSI-COV method the matrices are squared to find covariance functions. It also avoids the leakage effect and other issues associated with calculation of covariance. The SSI-DATA method can also yield prediction error and modal contributions which is not always the case with SSI-COV. However, the SSI-DATA algorithm requires more computation time compared to the calculation of the covariance functions in the SSI-COV method which can be efficiently computed by inverse Fourier transform of the power spectra by Fast Fourier transform techniques. 


\subsubsection{Spatial-domain algorithms}

\section{Frequency Domain Decomposition (FDD) and enhanced Frequency Domain Decomposition (eFDD)}

The derivations of spatial domain algorithms in OMA are inspired from the popular EMA algorithm of Complex Mode Indicator Function (CMIF). The so called Frequency Domain Decomposition method (FDD) was firstly presented in [37]. The method aims to be a simple and user-friendly technique allowing at the same time the separation of closely spaced modes and the identification of modal damping ratios. It is a frequency domain non-parametric method that utilizes output spectrum matrices estimated by the Welch method [38].

The derivations starts from the modal decomposition of transfer function and the relation between spectrum and transfer function presented in chapter 2 . For the sake of completeness, these two equations are restated here:

$$
\begin{aligned}
H(\omega) & =V_{c}\left(s I_{n}-\Lambda_{c, m}\right)^{-1} L_{c, m}^{T} \\
\mathrm{~S}_{\mathrm{yy}}(\omega) & =H(\omega) \mathrm{S}_{\mathrm{uu}} \mathrm{H}(\omega)^{H}
\end{aligned}
$$

where $V_{c}$ is expressed as follows (Equation 2-17)

$$
V_{c}=C_{c} \Psi
$$

And $\Lambda_{c, m}, L_{c, m}$ are as follows

$$
\begin{aligned}
& \Lambda_{c, m}=\left[\begin{array}{ll}
\Lambda & \\
& \Lambda^{*}
\end{array}\right] \\
& L_{c, m}^{T}=\left[\begin{array}{l}
L_{m}^{T} \\
L_{m}^{H}
\end{array}\right]
\end{aligned}
$$

where $\Lambda$ is a diagonal matrix containing system poles and $L_{m}^{T}$ is the participation matrix in modal space introduced in Section 2.5. 
Using these two equations the following output spectrum is obtained:

$$
\mathrm{S}_{\mathrm{yy}}(\omega)=\mathrm{V}_{\mathrm{c}}\left(s I_{n}-\Lambda_{c, m}\right)^{-1} L_{c, m}^{T} S_{u m} L_{c, m}^{*}\left(s I_{n}-\Lambda_{c, m}\right)^{-1 *} V_{c}^{H}
$$

Under the assumption of uncorrelated input and orthogonal mode shapes, the participation factors are also orthogonal. Then Equation (3-34) can be simplified as follows

$$
S_{y y}(\omega)=V_{c} C(\omega) V_{c}^{H}
$$

where $C(\omega)$ is a diagonal matrix and each of the diagonal element depends of frequency and damping ratio of single mode of structure. Even if the inputs are correlated but modal inputs are uncorrelated $\left(L_{c, m}^{T} u(t)\right)$, the same simplification can be done. Moreover, for the lightly damped structures, in the neighborhood of the resonant frequencies, the simplification presented in Equation (3-35) is still approximately true even if there is some correlation between the inputs or the modal inputs, as it may occur for the wind dominant excitation. However, the orthogonality of the mode shapes has to be maintained at least between closely spaced modes [37].

On the other hand, the estimated full output spectrum $S_{y y}(\omega)$ using Welch method can be decomposed by SVD as follows:

$$
\mathrm{S}_{\mathrm{yy}}(\omega)=U \Sigma U^{H}
$$

where $U$ is a orthonormal matrix that contains the singular vectors of $S_{y y}(\omega)$ and $\Sigma$ is a diagonal matrix of corresponding singular values. Comparing Equation (335)and(3-36), the columns of $U$ corresponding to the larger singular values is a 
structural mode. The related singular values are associated with the ordinates of spectra of single degree of freedom system. The rank of spectrum matrix or significant nonzero singular values determines the number of contributing mode at a particular frequency.

The FDD algorithm gives the frequency and mode shapes but for damping estimation and also for more accurate estimation of modal frequency one has to utilize the eFDD algorithm [37]. In the eFDD algorithm power spectra of a SDOF system is identified around a peak of resonance (a peak in the SVD plot). A user defined Modal Assurance Criterion (MAC] [39] rejection level is applied to compare the singular vectors around a peak with the associated singular vector of the peak and the corresponding portion of the spectra is identified as SODF spectra. The MAC can be defined as a measure of correlation between two mode shapes $\left(\theta_{i}, \theta_{j}\right)$ and expressed as follows

$$
M A C_{\theta_{i} \theta_{j}}=\frac{\left(\theta_{i}^{T} \theta_{j}\right)^{2}}{\left(\theta_{i}^{T} \theta_{i}\right)\left(\left(\theta_{j}^{T} \theta_{j}\right)\right.}
$$

Next this SDOF spectrum is taken back to time-domain by IFFT and the related autocorrelation is obtained. As mentioned in chapter2, under the white noise input assumption, the correlations are like impulse responses, so the damping of the mode can be obtained by using the classical impulse response relation of a SDOF system:

$$
y(t)=a e^{-\xi_{k} 2 \pi f_{k} t} \sin \left(2 \pi f_{k} t\right)
$$


where $\xi_{k}, f_{k}$ are modal damping ratio and natural frequency respectively. Before estimating the damping, one can obtain an enhanced estimate of the frequency by determining zero crossing time of SDOF correlation function.

\subsubsection{Frequency-domain algorithms}

The use of frequency domain algorithms for OMA purposes is not very common due to the numerical conditioning of the Vandermonde matrix that arises due to the least square fitting of the non parametric spectrum to the parametric spectrum model. For application of frequency-domain algorithms in OMA application field, the 4-quadrant symmetry of the power spectra, as mentioned in chapter 2 , together with the higher order polynomial can also seriously affect the numerical conditioning [40].

\section{Least Square Complex Frequency-Domain (LSCF) Estimator}

Originally the Least Square Complex Frequency-Domain Estimator (LSCF) method was developed to find the initial guess for the iterative Maximum Likelihood (ML) method [41]. The method estimates so called common denominator transfer model introduced in chapter 2. Quickly it was found that these initial values yield almost accurate modal parameters with a very little computational effort [41], [42], [43]. The added advantage of this method compared to other modal parameter estimation (MPE) algorithm can be attributed to clear stabilization diagram which is obtained by proper parameter constraint. A detailed study of different variant of common denominator model is discussed in [43]. It was found that identified common denominator model closely fits the FRF estimated from the data but when 
a SVD is performed to convert it to modal model Equation (2-27), the quality of the fitted model severely degrades. Furthermore, in case of a common denominator model only pole information is available to construct the stabilization diagram. Neither mode shape nor participation factors are available at the first stage. This theoretical drawback can seriously affect the modal parameter estimation as closely spaced poles will erroneously show up as a single pole. These two limitations provide the motivation for the polyreference version of LSCF method.

Polyreference Weighted Least Squares Complex Frequency Domain Estimator

This algorithm is the right matrix fraction based polyreference variant of the LSCF algorithm in frequency domain. It is also known as LMS PolyMAX. The LMS PolyMAX method is the only discrete time frequency domain model available for OMA application. This method was introduced in [44] together with a simulated example of closely spaced modes. In [45] PolyMAX was validated with an industrial data set and finally this method was extended to cover the OMA application in [46] . The mathematical background of this algorithm is summarized here.

The modal decomposition of spectrum matrix can be written as (Equation 2-50)

$$
S_{y y}(\omega)=\sum_{k=1}^{n_{2}} \frac{\left\{v_{k}\right\} \times\left\langle g_{k}^{T}\right\rangle}{j \omega-\lambda_{k}}+\frac{\left\{v_{k}^{*}\right\} \times\left\langle g_{k}^{H}\right\rangle}{j \omega-\lambda_{k}^{*}}+\frac{\left\{g_{k}\right\} \times\left\langle v_{k}^{T}\right\rangle}{-j \omega-\lambda_{k}}+\frac{\left\{g_{k}^{*}\right\} \times\left\langle v_{k}^{H}\right\rangle}{-j \omega-\lambda_{k}^{*}}
$$

Under the assumption of stationary stochastic process, a spectrum is calculated as the Discrete Fourier Transform of correlation function. So the discrete time spectrum equals: 


$$
\mathrm{S}_{\mathrm{yy}}(\omega)=\sum_{i=-\infty}^{\infty} R_{i} e^{-j \omega i \Delta t}
$$

where $R_{i}$ is output covariance calculated at lag time $i$ defined in Equation (3-23). In case of a stationarity process, the following property holds [24]:

$$
R_{-i}=R_{i}^{T}
$$

So, the spectrum can be written as:

$$
S_{y y}(\omega)=S_{y y}^{+}(\omega)+\left(S_{y y}^{+}(\omega)\right)^{H}
$$

where $S_{y y}^{+}(\omega)$, the "positive power spectra" is defined as [15]:

$$
S_{y y}^{+}(\omega)=\frac{R_{o}}{2}+\sum_{i=1}^{\infty} R_{i} e^{-j \omega t \Delta t}
$$

However as mentioned earlier, under the flat input spectrum assumption, the correlation function is similar to impulse response function. So just like an impact testing it seems logical to apply exponential window before taking the DFT Equation (3-39). This window is utilized to reduce the effects of the higher variance associated with the higher lag time of estimated correlation. Moreover, the effect of an exponential window on pole estimation can be corrected. This is not the case when a Hanning window is used; such a window always leads to a bias estimate of damping. Thus the weighted estimated spectrum is:

$$
\mathrm{S}_{\mathrm{yy}}(\omega)=\sum_{i=-L}^{L} w_{i} R_{i} e^{-j \omega i \Delta t}
$$


where $L$ is the maximum lag time at which correlations are estimated. It has been shown that in the context of modal analysis it is possible to simply calculate the half spectra of the system [46] :

$$
\mathrm{S}_{\mathrm{yy}}^{+}(\omega)=\frac{w_{0} R_{0}}{2}+\sum_{i=1}^{L} w_{i} R_{i} e^{-j \omega i \Delta t}
$$

It has been shown in [19] that the modal decomposition of the half spectra contains first two terms of Equation 2-50:

$$
S_{y y}^{+}(\omega)=\sum_{k=1}^{n_{2}} \frac{\left\{v_{k}\right\} \times<g_{k}^{T}>}{j \omega-\lambda_{k}}+\frac{\left\{v_{k}^{*}\right\} \times<g_{k}^{H}>}{j \omega-\lambda_{k}^{*}}
$$

The added advantage is that models of lower order models can be fitted without the affecting the quality. The positive power spectra can then be modeled as a right matrix-fraction model as follows (Equation 2-38):

$$
S_{y y}^{+}(\omega)=B(\omega) A(\omega)^{-1}
$$

where $\mathrm{B}(\omega)$ is the complex $I \times r$ numerator matrix, and $\mathrm{A}(\omega)$ is the complex $r \times r$ denominator matrix, where $l$ is the number of outputs of the system, and $r$ is the number of selected reference sensors. Each row of the model can be formulated as:

$$
S_{y y}^{+}(\omega)_{0}=B(\omega)_{0} A(\omega)^{-1}
$$

where $B(\omega)_{0}$ and $A(\omega)$ are formulated as follows:

$$
\begin{aligned}
& B(\omega)_{0}=\sum_{i=0}^{n_{\max }} z_{i}(\omega) \beta_{o i} \\
& A(\omega)=\sum_{i=0}^{n_{\max }} z_{i}(\omega) \alpha_{i}
\end{aligned}
$$


where $z_{1}$ is the basis function:

$$
z_{i}(\omega)=e^{j \omega i \Delta t}
$$

The coefficients can be written as follows:

$$
\beta_{0}=\left(\begin{array}{c}
\beta_{00} \\
\beta_{01} \\
\beta_{02} \\
\vdots \\
\beta_{0 n}
\end{array}\right), \alpha=\left(\begin{array}{c}
\alpha_{0} \\
\alpha_{1} \\
\alpha_{2} \\
\vdots \\
\alpha_{n}
\end{array}\right), \theta=\left(\begin{array}{c}
\beta_{1} \\
\beta_{2} \\
\vdots \\
\beta_{1} \\
\alpha
\end{array}\right)
$$

where $\beta_{o}$ is an $\left(n_{\max }+1\right) \times r$ matrix which can be constructed for all outputs of the system from $0=1 \rightarrow 1, \alpha$ is an $r\left(n_{\max }+1\right) \times r$ matrix and $\theta$ is an $(l+r)\left(n_{\max }+1\right) \times r$ matrix.

Equation (3-46) must be solved in order to determine all the values of $\beta$ and $\alpha$, or in this case $\theta$. This can be achieved by formulating a non-linear least squares equation and minimizing the associated errars as follows:

$$
\begin{aligned}
& \varepsilon_{0}^{\mathrm{N} L S}\left(\omega_{i}, \theta\right)=w_{0}\left(\omega_{i}\right)\left[S_{0}\left(\omega_{i}, \theta\right)-S_{0}\left(\omega_{i}\right)\right] \\
& \varepsilon_{0}^{N L S}\left(\omega_{i}, \theta\right)=w_{0}\left(\omega_{i}\right)\left[\beta_{0}\left(\omega_{i}, \theta\right) A^{-1}\left(\omega_{i}, \alpha\right)-S_{0}\left(\omega_{i}\right)\right]
\end{aligned}
$$

where $w_{0}\left(\omega_{1}\right)$ is a weighting function incorporated to account for the location of the measurements defined as [43]:

$$
w_{o}\left(\omega_{i}\right)=\frac{\left|S_{y y}^{+}\left(\omega_{i}\right)\right|}{\operatorname{var}\left(S_{y y}^{+}\left(\omega_{i}\right)\right)}
$$

Equation (3-50) is combined for all outputs and frequencies in the following cost function:

$$
\mathrm{I}^{\mathrm{NLS}}(\theta)=\sum_{0=1}^{1} \sum_{\mathrm{i}=1}^{\mathrm{N}_{\mathrm{f}}} \operatorname{tr}\left[\left(\varepsilon_{0}^{\mathrm{NL} S}\left(\omega_{1}, \theta\right)\right)^{\mathrm{H}} \varepsilon_{0}^{\mathrm{NLS}}\left(\omega_{i}, \theta\right)\right]
$$


In order to approximate this non-linear problem, the non-linear least squares problem of Equation (3-50), is multiplied by the numerator matrix A, resulting in a linear least squares problem as follows:

$$
\begin{aligned}
& \varepsilon_{0}^{L S}\left(\omega_{i}, \theta\right)=w_{0}\left(\omega_{i}\right)\left[B_{0}\left(\omega_{i}, \theta\right)-S_{0}\left(\omega_{i}\right) A\left(\omega_{i}, \alpha\right)\right] \\
& \varepsilon_{0}^{i S}\left(\omega_{i}, \theta\right)=w_{0}\left(\omega_{i}\right) \sum_{i=0}^{n_{\text {max }}}\left[z_{i}\left(\omega_{i}\right) \beta_{o k}-z_{1}\left(\omega_{i}\right) S_{0}\left(\omega_{i}\right) \alpha_{1}\right]
\end{aligned}
$$

These equation errors can now be combined in a single matrix consisting of every value of $\omega$ to be taken into account:

$$
E_{0}^{L S}(\theta)=\left[\begin{array}{c}
\varepsilon_{0}^{L S}\left(\omega_{1}, \theta\right) \\
\varepsilon_{0}^{L S}\left(\omega_{2}, \theta\right) \\
\vdots \\
\varepsilon_{0}^{L S}\left(\omega_{\left.N_{f^{\prime}}, \theta\right)}\right.
\end{array}\right]=\left[\begin{array}{ll}
X_{0} & Y_{0}
\end{array}\right]\left[\begin{array}{c}
\beta_{0} \\
\alpha
\end{array}\right]
$$

where $X_{0}$ and $Y_{o}$ are defined as follows:

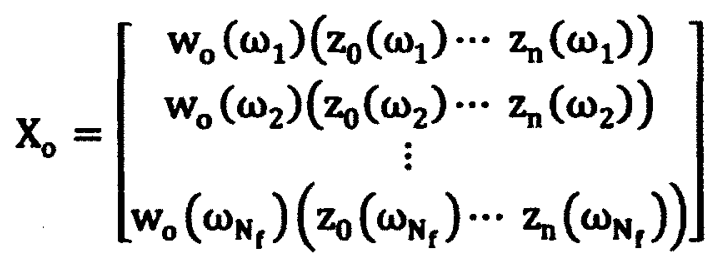

$$
\begin{aligned}
& Y_{0}=\left[\begin{array}{ccc}
-w_{0}\left(\omega_{1}\right)\left(z_{0}\left(\omega_{1}\right)\right. & \cdots & \left.z_{n}\left(\omega_{1}\right)\right) \otimes S_{0}\left(\omega_{1}\right) \\
-w_{0}\left(\omega_{2}\right)\left(z_{0}\left(\omega_{2}\right)\right. & \cdots & \left.z_{n}\left(\omega_{2}\right)\right) \otimes S_{0}\left(\omega_{2}\right) \\
& \vdots \\
-w_{0}\left(\omega_{N_{f}}\right)\left(z_{0}\left(\omega_{N_{f}}\right) \cdots\right. & \left.z_{n}\left(\omega_{N_{f}}\right)\right) \otimes S_{o}\left(\omega_{N_{f}}\right)
\end{array}\right]
\end{aligned}
$$

where $\otimes$ is defined as the Kronecker product, which is defined as

$$
A \otimes B=\left[\left[\begin{array}{ccc}
A_{11} B & \cdots & A_{1 n} B \\
\vdots & \ddots & \vdots \\
A_{m 1} B & \cdots & A_{m n} B
\end{array}\right]\right]
$$


where $A$ is an $m \times n$ matrix and $B$ is a $c \times d$ matrix, resulting in an $m c \times n d$ matrix. The cost function of Equation (3-53) can now be formulated based on the linearised equation errors:

$$
\mathrm{l}^{L S}(\theta)=\sum_{0=1}^{1} \sum_{k=1}^{N_{f}} \operatorname{tr}\left[\left(\varepsilon_{0}^{L S}\left(\omega_{k}, \theta\right)\right)^{H} \varepsilon_{0}^{L S}\left(\omega_{k}, \theta\right)\right]
$$

Minimizing this cost function results in a weighted linear least square problem. Using Equation (3-54) and (3-55), the cost function can be written as:

$$
\begin{aligned}
& l^{L S}(\theta)=\sum_{0=1}^{1} \operatorname{tr}\left[\left(E_{0}^{L S}(\theta)\right)^{\mathrm{H}} E_{0}^{L S}(\theta)\right] \\
& l^{L S}(\theta)=\sum_{0=1}^{1} \operatorname{tr}\left[\left(\begin{array}{ll}
\beta_{0}^{T} & \alpha^{T}
\end{array}\right)\left(\begin{array}{l}
X_{0}^{H} \\
Y_{0}^{H}
\end{array}\right)\left(\begin{array}{ll}
X_{0} & Y_{0}
\end{array}\right)\left(\begin{array}{c}
\beta_{0} \\
\alpha
\end{array}\right)\right. \\
& \mathrm{l}^{\mathrm{LS}}(\theta)=\operatorname{tr}\left(\theta^{\mathrm{T}} \mathrm{J}^{\mathrm{T}} \mathrm{J} \theta\right)
\end{aligned}
$$

where $J \in \mathbb{C}^{\mathbb{I N}_{f} \times(1+r)(n \max +1)}$ is the Jacobian matrix defined as:

$$
J=\left[\begin{array}{ccccc}
\mathrm{X}_{1} & 0 & \ldots & 0 & \mathrm{Y}_{1} \\
0 & \mathrm{X}_{2} & \cdots & 0 & \mathrm{Y}_{2} \\
& \vdots & \ddots & & \vdots \\
0 & 0 & \cdots & \mathrm{X}_{1} & \mathrm{Y}_{1}
\end{array}\right]
$$

It has been shown [46] that real part of $\mathrm{H}^{\mathrm{H}} \mathrm{J}$ can be utilized in the cost function in case of real valued coefficients $\theta$ :

$$
\mathrm{l}^{\mathrm{LS}}(\theta)=\operatorname{tr}\left(\theta^{\mathrm{T}} \operatorname{Re}\left(J^{\mathrm{H}} \mathrm{J}\right) \theta\right)
$$

where $\operatorname{Re}\left({ }^{\mathrm{H}}\right)$ is defined as: 


$$
\operatorname{Re}\left(J^{H} \mathrm{~J}\right)=\left[\begin{array}{ccccccc}
\mathrm{R}_{1} & 0 & & & 0 & S_{1} \\
0 & R_{2} & \cdots & & 0 & S_{2} \\
& \vdots & & \ddots & & & \vdots \\
0 & 0 & & R_{1} & S_{1} \\
S_{1}^{T} & S_{2}^{T} & \cdots & S_{1}^{T} & \sum_{0=1}^{I} T_{0}
\end{array}\right]
$$

where $R_{0}, S_{0}$, and $T_{0}$ are defined as follows:

$$
\begin{aligned}
& R_{o}=\operatorname{Re}\left(X_{0}^{H} X_{0}\right) \\
& S_{0}=\operatorname{Re}\left(X_{0}^{H} Y_{0}\right) \\
& T_{o}=\operatorname{Re}\left(Y_{o}^{H} Y_{0}\right)
\end{aligned}
$$

Now the cost function of Equation (3-60) is minimized by setting the partial derivatives with respect to $\theta$ equal to zero:

$$
\begin{aligned}
& \frac{\partial l^{L S}(\theta)}{\partial \beta_{0}}=2\left(R_{0} \beta_{o}+S_{0} \alpha\right)=0 \\
& \frac{\partial l^{L S}(\theta)}{\partial \alpha}=2 \sum_{0=1}^{l}\left(S_{0}^{T} \beta_{o}+T_{0} \alpha\right)=0
\end{aligned}
$$

Equation (3-65) can be written synthetically using Equations (3-59) and (3-49)

$$
2 \operatorname{Re}\left(J^{H}\right) \theta=0
$$

As the denominator matrix $\alpha$ contains the information for the poles and operational reference factors, $\beta_{o}$ can be eliminated from Equation (3-65) as follows:

$$
\beta_{0}=-R_{0}^{-1} S_{0} \alpha
$$

which results in: 


$$
\begin{aligned}
& 2 \sum_{0=1}^{1}\left(S_{0}^{T}\left(-R_{0}^{-1}\right) S_{0}+T_{0} \alpha\right)=0 \\
& \left(2 \sum_{0=1}^{1} T_{0}-S_{0}^{T} R_{0}^{-1} S_{0}\right) \alpha=0 \\
& M \alpha=0
\end{aligned}
$$

A $n_{\max }$ order right matrix fraction based PolyMAX model will yield $r \times n_{\max }$ poles. In order to efficiently construct the necessary stabilization diagram needed to determine the stable physical poles of the system, the least square problem of Equation (3-68) is formulated for the maximum model order $\mathbf{n}_{\max }$. Following this formulation, submatrices of appropriate dimensions can be constructed for the model order $n$. In order to solve Equation (3-68) a constraint must be placed on the system of equations to exclude the trivial solution of $\alpha=0$. The selection of this constraint plays an important role in the clarity of the resulting stabilization diagrams. Figure 3-1 demonstrates the clean well-defined diagrams provided when the constraint $\alpha_{1}=I_{r}$ is applied. This constraint results in negative damping values for the mathematical poles, allowing for their exclusion prior to plotting. A constraint of $\alpha_{n+1}=$ Ir does not provide a distinction between physical and mathematical poles with regards to damping ratios, thus all poles must be plotted, resulting in an indistinguishable diagram as shown in Figure 3-2. A detail study of the effect of the parameter constraint on the quality of the stabilization diagram can be found [47]. 


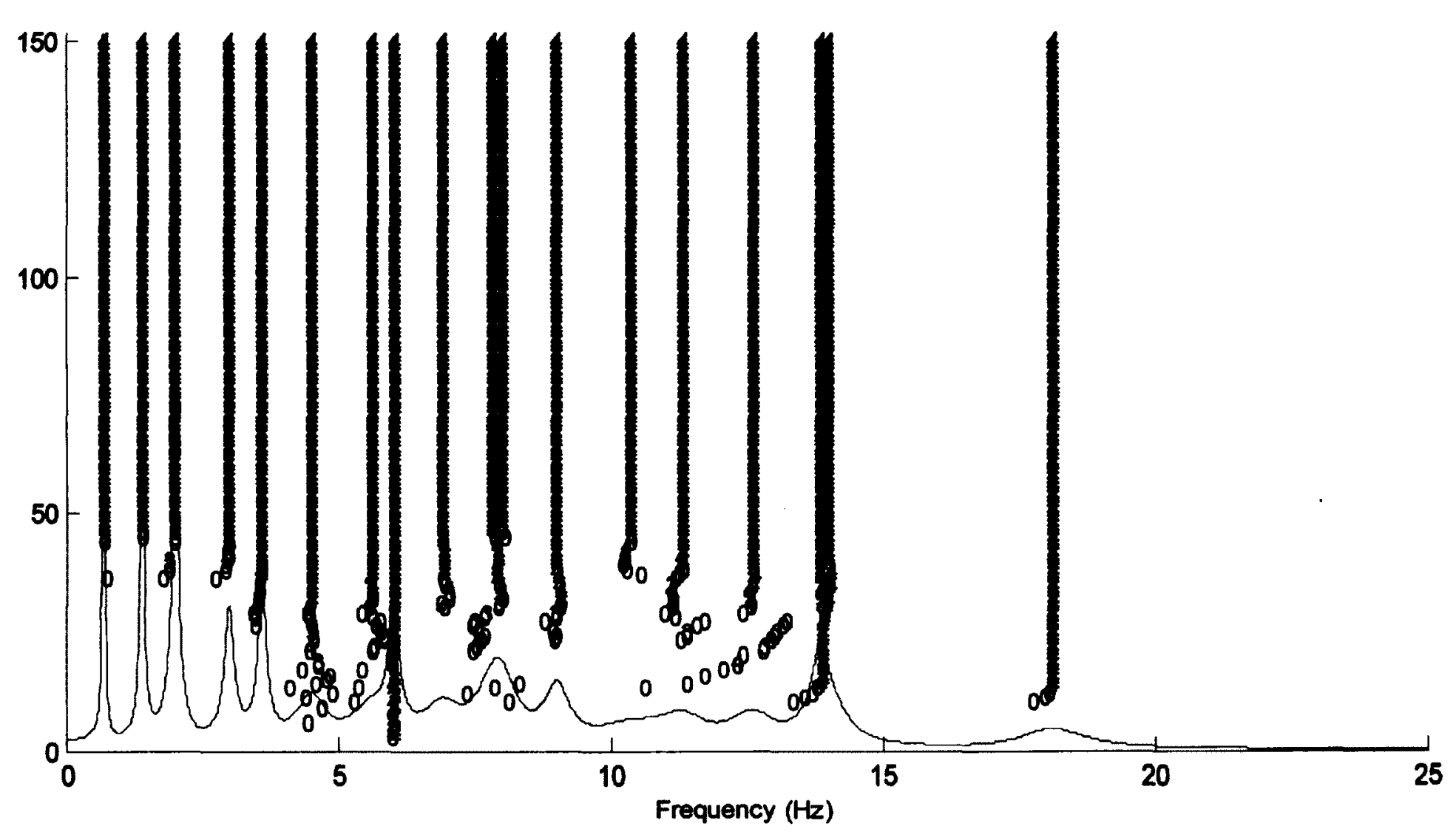

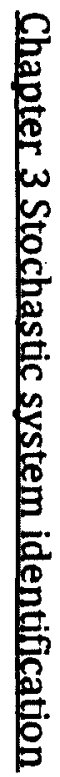

Figure 3-1: Stabilization diagram based on p-LSCF estimator with $\alpha_{1}=l_{r}$ constraint 


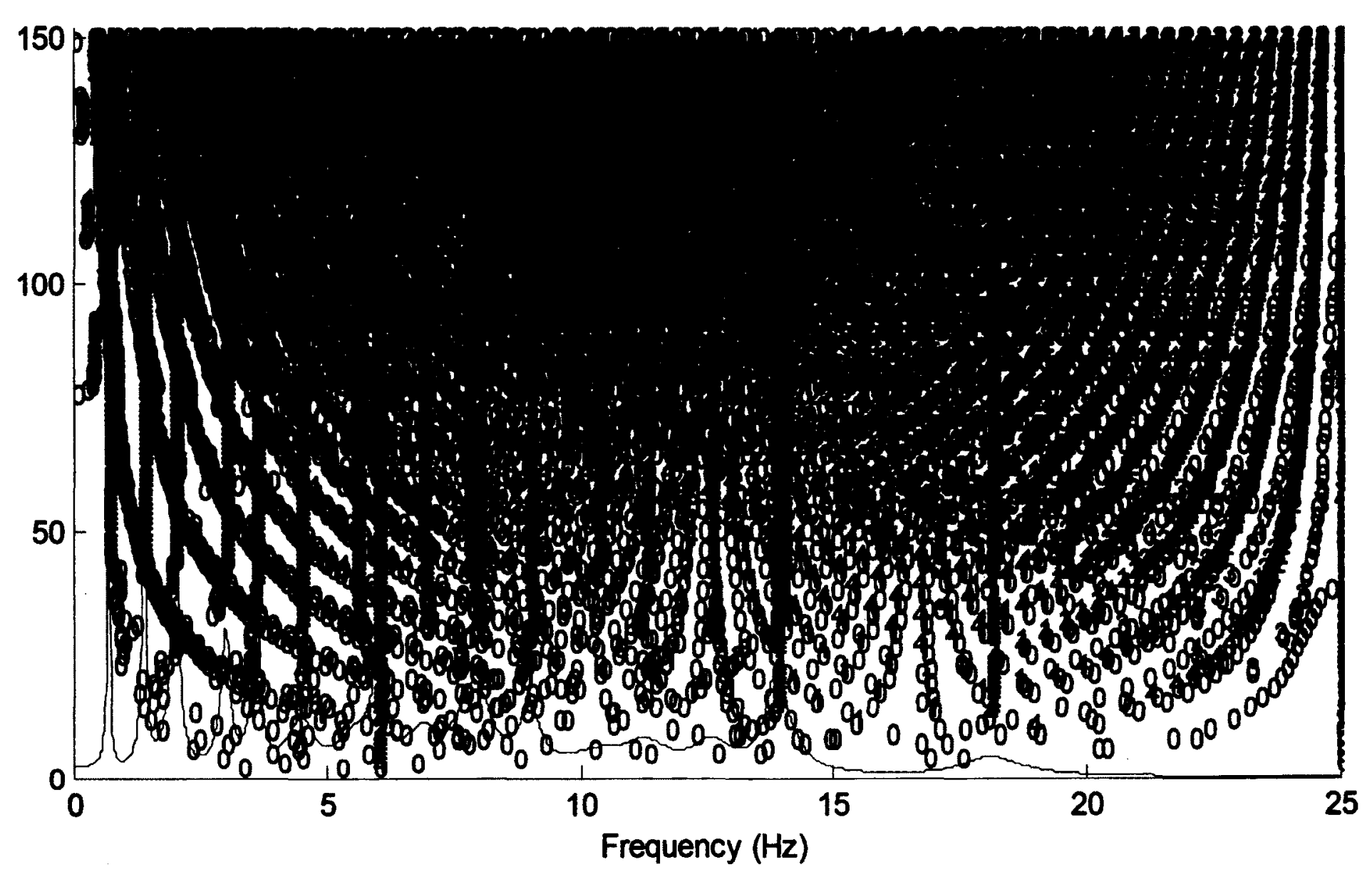

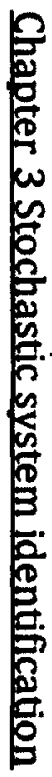

Figure 3-2: Stabilization diagram based on p-LSCF estimator with $\alpha_{n+1}=I_{r}$ constraint 
Based on the constraint $\alpha_{1}=k$, the following problem can be formulated:

$$
\mathbf{A x}=\mathbf{B}
$$

where $A=M(r+1: r(n+1), r+1: r(n+1))$ and $B=-M(r+1: r(n+1), 1: r)$.

Upon solving Equation (3-69) the least-squares estimate of Equation (3-68) can be written as follows:

$$
a_{L S}=\left[\begin{array}{l}
I_{r} \\
x
\end{array}\right]
$$

The discrete-time poles are then found as the eigenvalues of the following companion matrix:

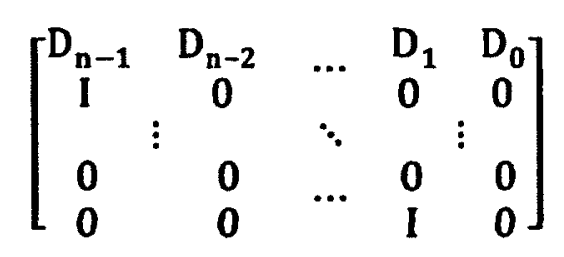

where $D_{j}=-\hat{\alpha}_{n}^{-1} \alpha_{j}$.

The last $m$ rows of the eigenvector matrix correspond to the operational reference factors. The interpretation of stabilization diagram yields a set of stable poles and reference factors. The mode shapes can be obtained from Equation (3-45):

$$
S_{y y}^{+}(\omega)=\sum_{k=1}^{n_{2}} \frac{\left\{v_{k}\right\} \times<l_{k}^{T}>}{j \omega-\lambda_{k}}+\frac{\left\{v_{k}^{*}\right\} \times<l_{k}^{H}>}{j \omega-\lambda_{k}^{*}}+\frac{L R}{j \omega}+j \omega U R
$$

where $L R, U R \in \mathbb{R}^{1 \times r}$ respectively the lower and upper residuals and is used to model the effect of out of band modes in the considered frequency band. The only unknown in this equation is mode shapes $\left\{v_{k}\right\}$. They are easily obtained by solving this equation in a linear least square sense. This step is commonly known as Least Square Frequency Domain (LSFD) method [3]. 


\section{Maximum Likelihood (ML) based algorithms}

Maximum Likelihood (ML) identification is an optimization based method that estimates the parameters of a model by minimizing an error norm. A discussion on the use of the ML estimator to identify parametric frequency-domain models can be found in [48] [49]. This algorithm involves estimation of the same model as represented by Equation (3-45) by Gauss-Newton optimization of the negative loglikelihood function given as

$$
\mathrm{l}_{\mathrm{ML}}(\theta)=\sum_{\mathrm{o}=1}^{l} \sum_{\mathrm{f}=1}^{\mathrm{m}} \sum_{\mathrm{f}=1}^{\mathrm{N}_{\mathrm{f}}} \frac{\left|\mathrm{S}_{\mathrm{oi}}\left(\theta, \omega_{\mathrm{f}}\right)-\mathrm{S}_{\mathrm{oi}}\left(\omega_{\mathrm{f}}\right)\right|^{2}}{\operatorname{var}\left(\mathrm{S}_{\mathrm{oi}}\left(\omega_{\mathrm{f}}\right)\right)}
$$

Thus ML method results in equations that are non-linear in the unknown system parameters. Since the ML estimator algorithm uses an optimization scheme, it is iterative and requires good starting values. However, it seems that these drawbacks have been overcome and in [41] ML identification is used to find the modal parameters of a structure from a large and noisy data set. Originally intended for application to FRFs, the method was extended to use spectra as primary data, so that it also could be used in output-only cases [19]. 


\subsection{OMA associated data processing}

The identification methods presented in the previous sections are based on discrete time series of measured accelerations. These discrete time series have to be processed accordingly prior to their use by the identification algorithm.

\section{Correlation estimate}

The unbiased discrete-time correlation estimate between the response signals $\left\{y_{k}\right\} \in R^{N_{o} \times 1}$ for $(\mathrm{k}=0, \ldots, \mathrm{N}-1)$ and the reference output signals $\left\{y_{m}^{\text {ref }}\right\} \in R^{N_{\text {ref }} \times 1}$ is given by:

$$
\begin{array}{ll}
R_{k}=\frac{1}{N-k} \sum_{m=0}^{N-k-1}\left\{y_{k+m}\right\}\left\{y_{m}\right\}_{r e f}^{T} & \text { for } 0 \leq k<N-1 \\
R_{k}=\frac{1}{N-|k|} \sum_{k=0}^{N-|k|-1}\left\{y_{m}\right\}\left\{y_{m+|k|}\right\}_{r e f}^{T} & \text { for }-(M-1) \leq k<0
\end{array}
$$

with $k$ the correlation lag time. The biased correlation estimate uses $1 / N$ rather than $1 /(N-k)$. It is shown that linear correlation can be efficiently calculated by discrete Fourier transform using a technique called zero-padding [50] [9]. In this process, the output signal is divided in $N_{b}$ data blocks. Each of the $N_{b}$ adjacent data blocks, each $\mathrm{N}$ output samples, is extended by an additional block of $\mathrm{N}$ zeros. In other words, new blocks $y_{b}(k)$ of length $2 \mathrm{~N}$ are created such that 


$$
\begin{array}{ll}
y_{b}(k)=y_{b}(k) & \text { for } 0 \leq k \leq N-1 \\
y_{b}(k)=0 & \text { for } N-1<k \leq 2 N-1
\end{array}
$$

Now, consider the DFT without applying a window

$$
Y_{b}\left(\omega_{k}\right)=\sum_{m=0}^{2 N-1} y_{b}(m) z_{k}^{-m}
$$

And calculating the corresponding power spectra by

$$
S_{y y}^{r e f}\left(\omega_{k}\right)=\frac{1}{N_{b}} \sum_{b=1}^{N_{b}} Y_{b}\left(\omega_{k}\right) Y_{b}^{r e f, H}\left(\omega_{k}\right) \in C^{N_{o} \times N_{r e f}}
$$

Then it is shown in [50] that the IDFT of these power spectra followed by a correction given by Equation (3-79) results in the linear correlation function $R(i)$

$$
\begin{gathered}
R_{i}^{c}=\frac{1}{N} \sum_{m=0}^{2 N-1} S_{y y}^{r e f}\left(\omega_{k}\right) z_{m}^{k} \\
R_{i}=\frac{N}{N-k} R_{i}^{c}
\end{gathered}
$$

In fact this correlation $R_{\mathfrak{l}}$ is exactly the same correlation function as the one by averaging the correlation functions from the $N_{b}$ adjacent blocks, but the use of the DFT and IFT functions speeds up the calculation time. This procedure is shown in Figure 3-3

\section{Spectrum Estimation}

DFT of correlation sequence is defined as spectrum of the signal. Probably the most widely used non parametric estimate of spectrum is Periodogram power density estimator, also known as the Welch estimator [38]. The basic idea of the periodogram estimator is to divide the response signals in $N_{b}$ blocks of equal length. 
Next, the Fourier spectra, for each block weighted with a time window $w(m)$, are computed as:

$$
Y_{b}\left(\omega_{k}\right)=\sum_{m=0}^{N-1} w(m) y_{b}(m) z_{k}^{-m}
$$

with $\mathrm{N}$ the number of time samples within a block. The time window $w(m)$ (e.g. Hanning, Hamming) typically reduces the influence of leakage. Since the window reduces the contribution of the data at the beginning and end of the record, introducing an overlap between the adjacent blocks in order to obtain a better contribution of each raw time data sample is needed. The power density spectra are given by:

$$
S_{y y}^{r e f}\left(\omega_{k}\right)=\frac{1}{N_{b}} \sum_{b=1}^{N_{b}} Y_{b, k} Y_{b, k}^{r e f, H} \in C^{N_{o} \times N_{\text {ref }}}
$$

The choice of the number of samples $\mathrm{N}$ within a block and thus the number of blocks $N_{b}$ is a trade-off between variance and bias on the estimated power spectra. Choosing a higher amount of data samples $\mathbf{N}$ and a lower amount of blocks $N_{b}$ reduces the effect of leakage but increases the stochastic uncertainty i.e. the variance on the estimated power spectra.

In the Correlogram approach the output spectrum is calculated from the DFT of the output correlation matrix. To reduce the effect of leakage the use of an adequate ( $2 \mathrm{~N}$ +1)-point time window $w$ symmetric around the origin is advised. This window reduces the effect of leakage and thus the bias error in the power spectra. So the spectrum with respect to a reference sensor is: 


$$
S_{y y}\left(\omega_{k}\right)=\sum_{m=-N}^{N} w(m) R(m) e^{j 2 \pi m k / N}
$$

The spectrum thus calculated has 4-quadrant symmetry (Equation 2-50). From the modal analysis point of view, it means more computational effort and less good numerical conditioning. Most importantly the power spectrum has both stable and unstable poles, thus has less interesting interpretation of the stabilization diagram. Keeping all these points in mind, it is advised to calculate the positive power spectrum which is equivalent to FRF modal decomposition (Equation (3-45). The estimation of the positive power spectra $S_{y y}^{+}(\omega)$ can be summarized as follows and is visually given by Figure 3-3

1. Divide the time records in $N_{b}$ adjacent blocks of $\mathrm{N}$ data samples. Extend each block with an additional $\mathrm{N}$ zeros.

2. Based on these extended blocks, calculate the power spectra with the periodogram method. The inverse Fourier transform of these power spectra results in the linear correlation functions.

3. The positive power spectra $S_{y y}^{+}(\omega)$ by considering only the first $N_{w}$ positive lags of the correlation function. 

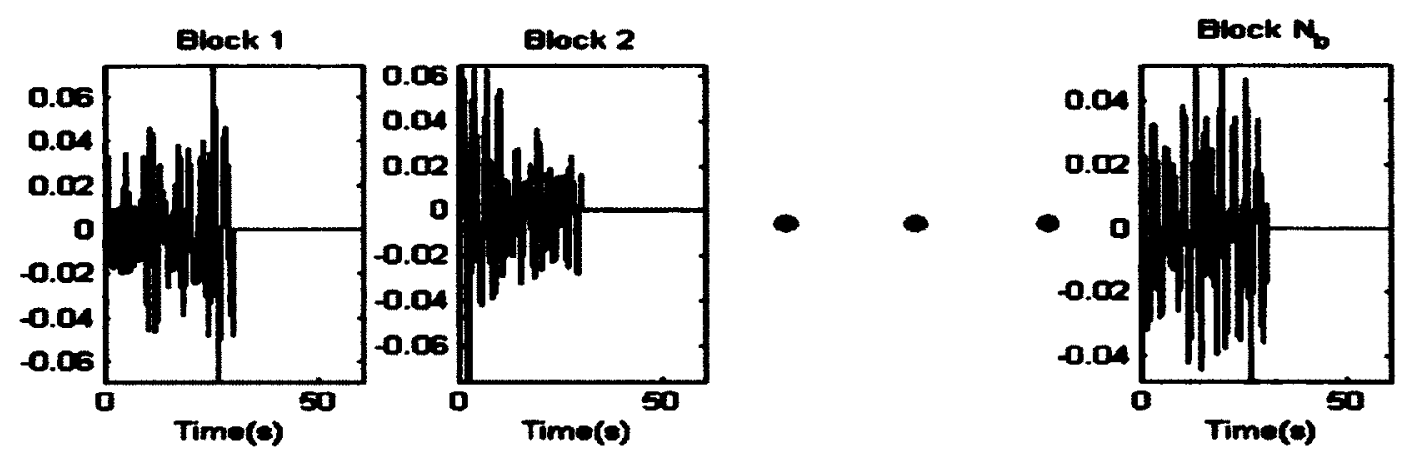

$\frac{0}{5}$
$\frac{0}{0}$
0
0
0
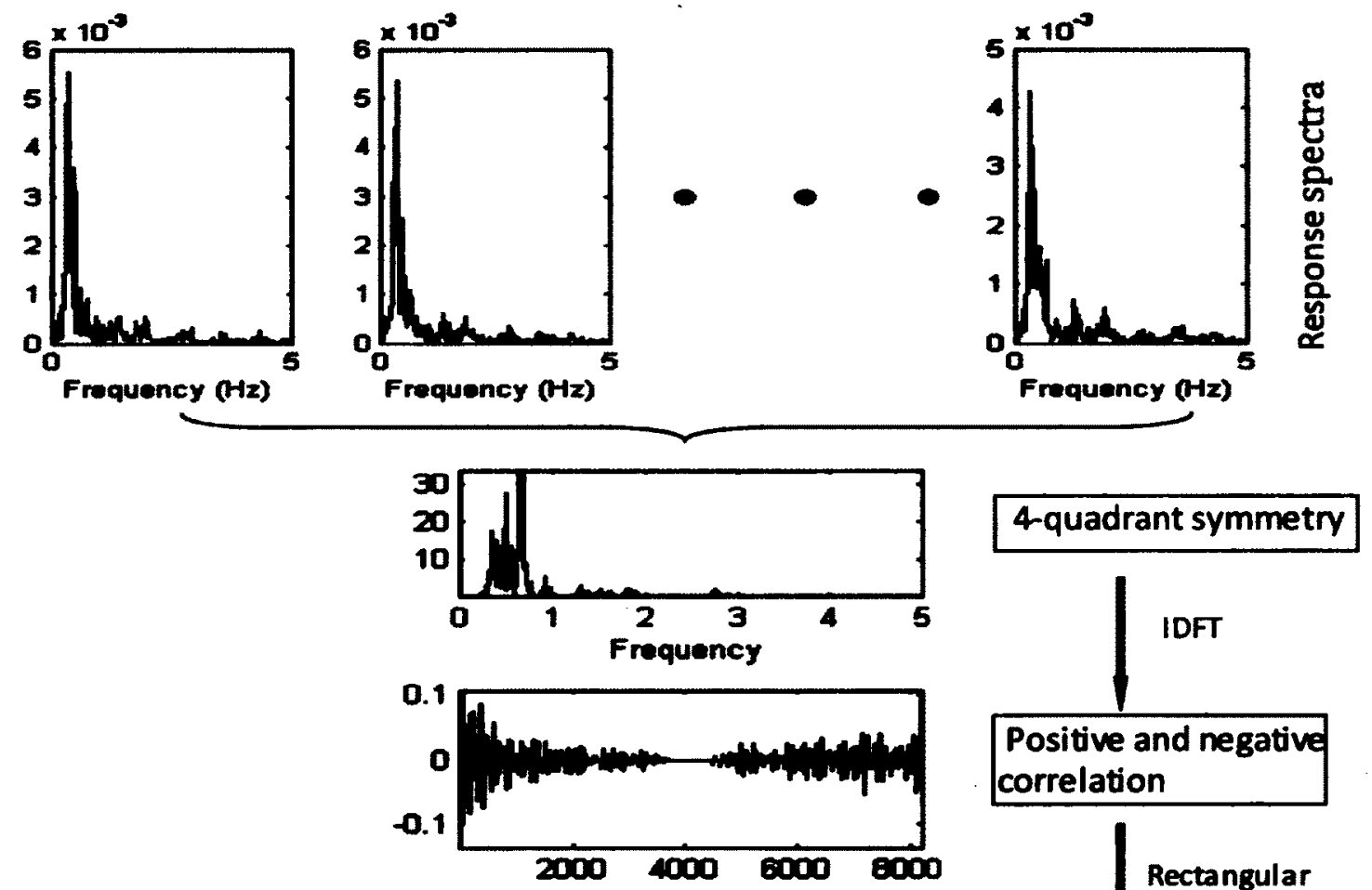

Positive and negative correlation

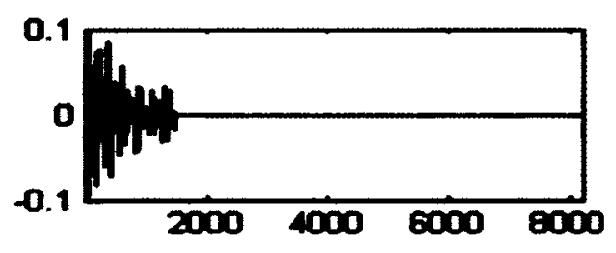

Rectangular window

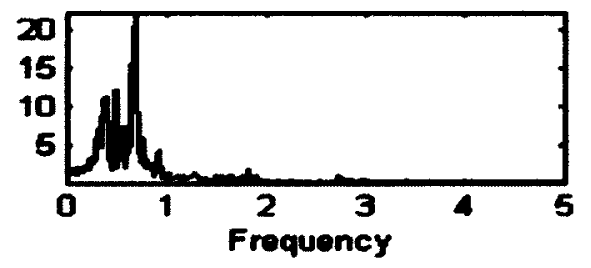

DFT

Figure 3-3: Schematic of positive power spectra estimation 


\section{Chapter 4 - Implementation of}

\section{OMA: Pros and Cons}

\subsection{Introduction}

This chapter starts with a brief overview of operational modal analysis (OMA) application in civil engineering. Based on the existing application examples, several issues with OMA applicability are presented followed by an up to date research work to overcome these limitations. Then a systematic comparison of identification algorithms in terms of modal parameter estimation is performed using a simulated example. The effects of violating the basic OMA assumptions are also explored in this study. It has been observed that damping estimate from OMA algorithms are not as reliable as frequency estimate and sometimes the errors can be significant. Thus an attempt has been made to identify the causes for damping inaccuracy and assess the currently available signal processing technique for correcting this problem. 


\subsection{OMA applications}

The difficulties encountered during attempts of Experimental modal analysis (EMA) algorithms for large complex structures such as buildings, bridges and airport drive the motivation of the development of Operational modal analysis (OMA) algorithms. In general, civil engineering structures are most popular application area of OMA as it is very difficult and expensive to excite the large structure with a hammer or shaker and to obtain the artificially induced vibration levels that exceed the natural vibration level due to ambient excitation. In current literature, there are some field application cases of OMA techniques to civil structure for parameter estimation. The Swiss Z24 highway bridge was tested considerably and data were collected under different loading scenarios including ambient loading conditions. Several OMA parameter estimation methods were applied to analyze the obtained data to compare the differences in the performance of the different OMA algorithms. The OMA methods considered in the studies include from simple peak picking to more advanced methods Stochastic Subspace Identification (SSI), Auto Regressive Moving Average (ARMAV) estimation using Prediction Error method (PEM) and Polyreference Least Square Complex Exponential (p-LSCE) methods. It is reported that all these methods yielded comparable modal parameters. The results are discussed in details in the reference [51]. In a follow-up study to this paper, the performance of OMA techniques are compared with the EMA methods [52]. In this study, seven research teams from around the world were invited to independently 
analyze the same set of measured vibration data using their own system identification methods of choice. This study provided insights to various OMA methods from a practical application point of view and the results corroborated that SSI method gave the most complete and consistent modal parameters estimation. It is worth noting that this technique performs very well at extracting closely spaced vibration modes. It was also suggested that if the structure has low natural frequencies, below $1 \mathrm{~Hz}$, then they are best excited by ambient sources or by drop weight excitation. In such cases use of a shaker as external excitation source might not be advantageous. Further, it is noted that high frequency modes are typically not well excited by ambient sources. In more recent field monitoring cases of long-term continuous structural health monitoring of critical bridges, ambient excitations are the only practical alternatives as the sources of vibration excitation to the structures [52]. Findings from the study on the ambient vibration behavior of the Heritage Court Tower 2 in Vancouver, Canada and the results are summarized in [53]. As in Z24 bridge study [51], this study also concluded that among the different algorithms used, the SSI method provides the most consistent modal parameter estimation results. Ambient vibration data from the Vasco Da Gamma Bridge were analyzed by Frequency Domain Decomposition (FDD) and SSI methods in reference [54] and it was stated that both the methods are more robust compared to traditional PeakPicking method.

Operational modal analysis (OMA) algorithms have also been used in Automo tive and Aerospace industry. In the study by Hermans and Van der Auweraer [55] p- 
LSCE and Canonical Variance Analysis (CVA) and Balance Realization (BR) variants of covariance based SSI (SSI-COV) algorithm were applied to identify the modal parameters from the data of the rear suspension system of a passenger car. The operational data in this case was collected by running the vehicle on a rough asphalt road at a speed on $50 \mathrm{~km} /$ hour. The main purpose of the study was to identify the source of a booming interior noise at around $80 \mathrm{~Hz}$. With the help of OMA tests, the first bending mode of the rear suspension twist beam was identified around 70-80 $\mathrm{Hz}$ which was the potential cause of the problem. This study mentioned that the mode was more easily identified by CVA and BR algorithms in comparison to p-LSCE [55]. This paper also presents a case study where OMA is applied to flight flutter test data to find out the robustness and suitability of these techniques to nonstationary data as those encountered in flight flutter testing. It is reported that SSICOV methods gave better results than the p-LSCE algorithm. Further in another study, flight flutter data was analyzed using least squares time and frequency domain algorithms as well as maximum likelihood (MLE) based approach [56]. It was observed that time domain LSCE does not perform as well as the other methods. Frequency domain PolyMAX is extended to output only cases in [46]. Both SSI and PolyMAX methods were applied to analyze to flight flutter test data and similar results are obtained, though it is mentioned that PolyMAX method can identify more stable poles compared to SSI method. The performance of PolyMAX is again compared with SSI by ambient vibration of stadium during a football game [57]. 


\subsection{Major issues of OMA}

Two major assumptions regarding OMA are as follows:

- The nature of the input force is assumed to be random, broadband and smooth indicating no poles or zeros in the frequency range of interest of the input power spectrum.

- The input on the structure is assumed to be uniformly distributed spatially. It is noted that the very assumptions that make OMA procedure possible are also the cause of its limitations. The lack of the input information of the external excitation applied to the structure affects the analysis processes in various stage of modal parameter estimation. These issues are briefly summarized here.

- From OMA application point of view, it is important to have sufficient excitation being provided by natural sources in order to get a good signal-to noise ratio of the vibration response data. In addition to this, longer time histories are required to compute accurate estimate of the response spectrum in the frequency domain, or correlation in the time domain compared to FRF estimate in EMA.

- From Natural Excitation Technique (NExT) point of view, any output measurements can be used as the reference sensor. The same can be said of the SSI or PolyMAX algorithm. To minimize the computational effort only a subset of sensors are chosen as reference sensors. This results in an important subjective step in the identification process requiring the judgment and experience of the 
analyst. Different selections of reference sensors may lead to slightly different identification results, including the omission or poor identification of important vibration modes. The criteria in selecting the reference outputs are analogous to the selection of input locations in traditional input-output modal testing.

- When confronted with large structures or when a high spatial resolution is required, measurement may not always be performed in all experimental DOFs at once. Therefore, schemes of moving multiple sensor setups are sometimes necessary. However, it is important to recognize that merging of data from different patches will fail if the response process is nonstationary for different records. A covariance matrix normalization technique has been developed to resolve this issue [58]. Interesting to note that normalization has the advantage of smoothing out the non-stationarity in the data. Two other approaches for patching non-stationary data are introduced in [18] for frequency domain OMA which is also applicable to time domain method.

- Most operational modal identification procedures, as in their counterparts in EMA use Ordinary Least Square solution to solve an over determined set of linear equations. Therefore bias error can occur due to noise and un-modeled effects even in the case of recently developed frequency domain PolyMAX method. Theoretically, bias can be reduced by using Total Least Square (TLS) or Partial Least Square (PLS) instead of the conventional Least Square (LS) approach. However, experiences have shown that the improvement is relatively little compared to the rather significant increases in computational effort 
required. In time domain PEM and in frequency domain MLE methods have the ability to extract the unbiased modal parameters with confidence level. However, as mentioned in Chapter 3 , these methods are computationally intensive, does not guarantee convergence and need an "initial" guess for the iterative search.

- Operational analysis assumes that the input signal is a white noise sequence. A violation of this assumption will lead to extra peaks in the cross power spectra that are not evidence of structural modes as discussed in Section 4.6. Clearly, these extra peaks need to be distinguished from the true structural modes. In most cases, some pre-knowledge about the working conditions of the inoperation structure is sufficient to discriminate these colored excitation poles from true physical modes. One of the simple ways to distinguish between a structural mode and harmonic excitation is that modes appearing due to harmonic excitation will have very low (near zero) damping [59]. An overview of other possible solutions is given in [60].

- As no input information is available, one cannot be sure whether all the modes of interest are excited or not. This is the basis of second assumption which states that excitation should be spatially complete.

- Mode shape is a description of the relative motion between degrees-of-freedom but the length of the mode vector can be arbitrarily selected. The modal scaling constant helps to preserve the proper scaling relationship between the mode shapes and the system modal mass, modal damping and modal stiffness. When 
mass matrix $[M]$ is available, the mode vectors can be scaled so that modal mass is unity. However, from an experimental viewpoint no mass matrix is available for computing the modal mass; even then the scaling factor can be computed if the complete loading information is available like in traditional EMA application cases [4]. A prime concern in OMA is that it does not allow mode shape scaling because of the lack of information of input excitation. To allow the identified modal model to be used for structural response simulation or modal updating, the scaling factor of the mode shape must be known. Also in health monitoring applications where damage is to be identified, the scaling factor of mode shape is important as several damage detection algorithms depend on the change of amplitude of modal co-ordinate. Some suggestions are provided for solving this problem in [61]. However, according to this method, knowledge of the full set of the vibration modes is needed. Recently a new approach based on repeated testing where the scaling factor can be obtained by mass change method is proposed [18]. The idea is based on the fact that if a small mass modification is performed in such a manner that mode shapes remain same but the natural frequency of the system changes slightly. If the mass modification matrix is proportional to the original mass matrix of the system, the error in scaling factor estimation tend to be diminished. However, in real applications, this is often not practically feasible. Thus in case where scaling factor is a necessary, the EMA techniques are preferred. 


\subsection{Numerical simulation}

Having discussed different identification algorithms and related issues with OMA application, next a comparative study is performed with an 8 DOF system (Figure 4-1) excited by "uncorrelated" random white noise at all DOF simultaneously. Mass and stiffness matrices of the simulated system are presented below. Damping is modeled as the special case of proportional damping.

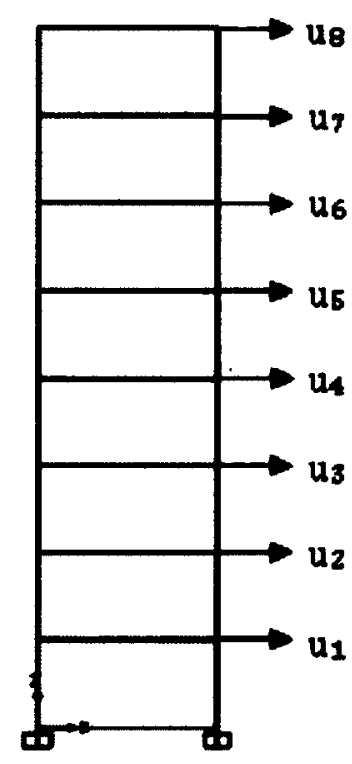

Figure 4-1: Simulated 8-dof system

$$
M=\left[\begin{array}{llllllll}
8 & 0 & 0 & 0 & 0 & 0 & 0 & 0 \\
0 & 8 & 0 & 0 & 0 & 0 & 0 & 0 \\
0 & 0 & 8 & 0 & 0 & 0 & 0 & 0 \\
0 & 0 & 0 & 8 & 0 & 0 & 0 & 0 \\
0 & 0 & 0 & 0 & 8 & 0 & 0 & 0 \\
0 & 0 & 0 & 0 & 0 & 4 & 0 & 0 \\
0 & 0 & 0 & 0 & 0 & 0 & 4 & 0 \\
0 & 0 & 0 & 0 & 0 & 0 & 0 & 4
\end{array}\right] \times 10^{6} \mathrm{Kg}
$$




$$
\mathrm{K}=\left[\begin{array}{rrrrrrrr}
2 & -1 & 0 & 0 & 0 & 0 & 0 & 0 \\
-1 & 1.5 & -0.5 & 0 & 0 & 0 & 0 & 0 \\
0 & -0.5 & 1 & -0.5 & 0 & 0 & 0 & 0 \\
0 & 0 & -0.5 & 1 & -0.5 & 0 & 0 & 0 \\
0 & 0 & 0 & -0.5 & 1 & -0.5 & 0 & 0 \\
0 & 0 & 0 & 0 & -0.5 & 1 & -0.5 & 0 \\
0 & 0 & 0 & 0 & 0 & -0 & 1 & -0.5 \\
0 & 0 & 0 & 0 & 0 & 0 & -0.5 & 0.5
\end{array}\right] \times 10^{3} \mathrm{KN} / \mathrm{mm}
$$

The system is converted to continuous time state space model. Simulated acceleration response of the system is measured by accelerometer sensors. Dimension of the state-space model order are $n=16$, the number of inputs $m=8$ and the number of outputs $l=8$. Assuming Zero-Order Hold (ZOH) on the inputs and a sampling period $\Delta t=0.01 \mathrm{~s}$, a discrete-time state-space model is obtained. Note that the sampling frequency $f_{s}=1 / \Delta t$ is chosen such that the Nyquist frequency $f_{N}=f_{s} / 2$ is well above the largest eigenfrequency: $f_{8}=3.248 \mathrm{~Hz}$ (see Table 4-1). The data was collected for 700s. The first input signal is shown in Figure 4-2 and the first output signal is shown in Figure 4-3. A typical impulse response, computed from the discrete state-space model according to Equation $(2-18)$ is shown in Figure 4-4.

Table 4-1: Modal frequencies and damping of analytical 8 DOF system

\begin{tabular}{ccc}
\hline$\#$ & Eigenfrequency, $f_{i}(\mathrm{~Hz})$ & Damping Ratio (\%) \\
\hline 1 & 0.324 & 1.3000 \\
2 & 0.869 & 1.0392 \\
3 & 1.320 & 1.2386 \\
4 & 1.779 & 1.5118 \\
5 & 2.220 & 1.8000 \\
6 & 2.485 & 1.9798 \\
7 & 2.998 & 2.3368 \\
8 & 3.248 & 2.5132 \\
\hline
\end{tabular}




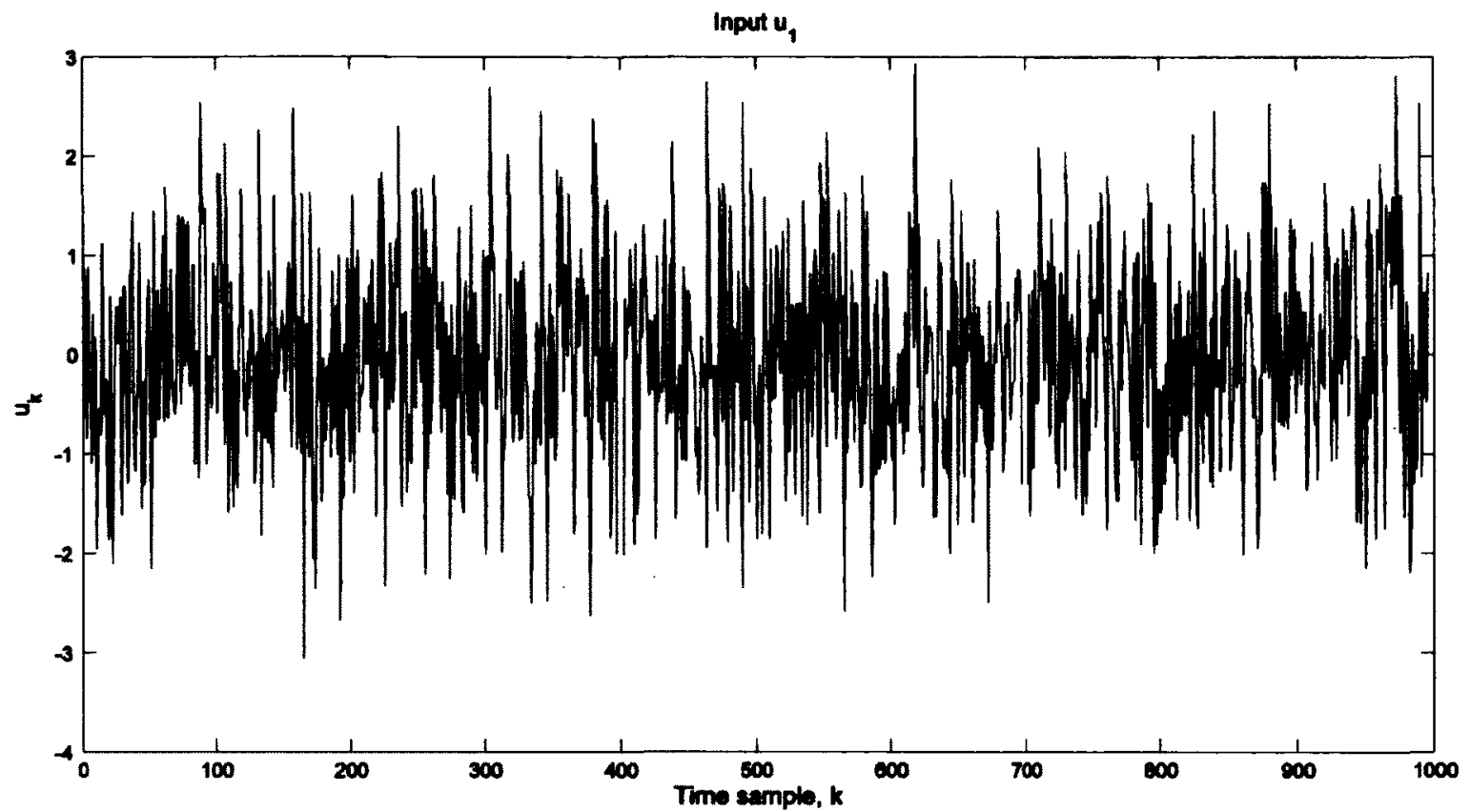

Figure 4-2: Part of the first white input signal

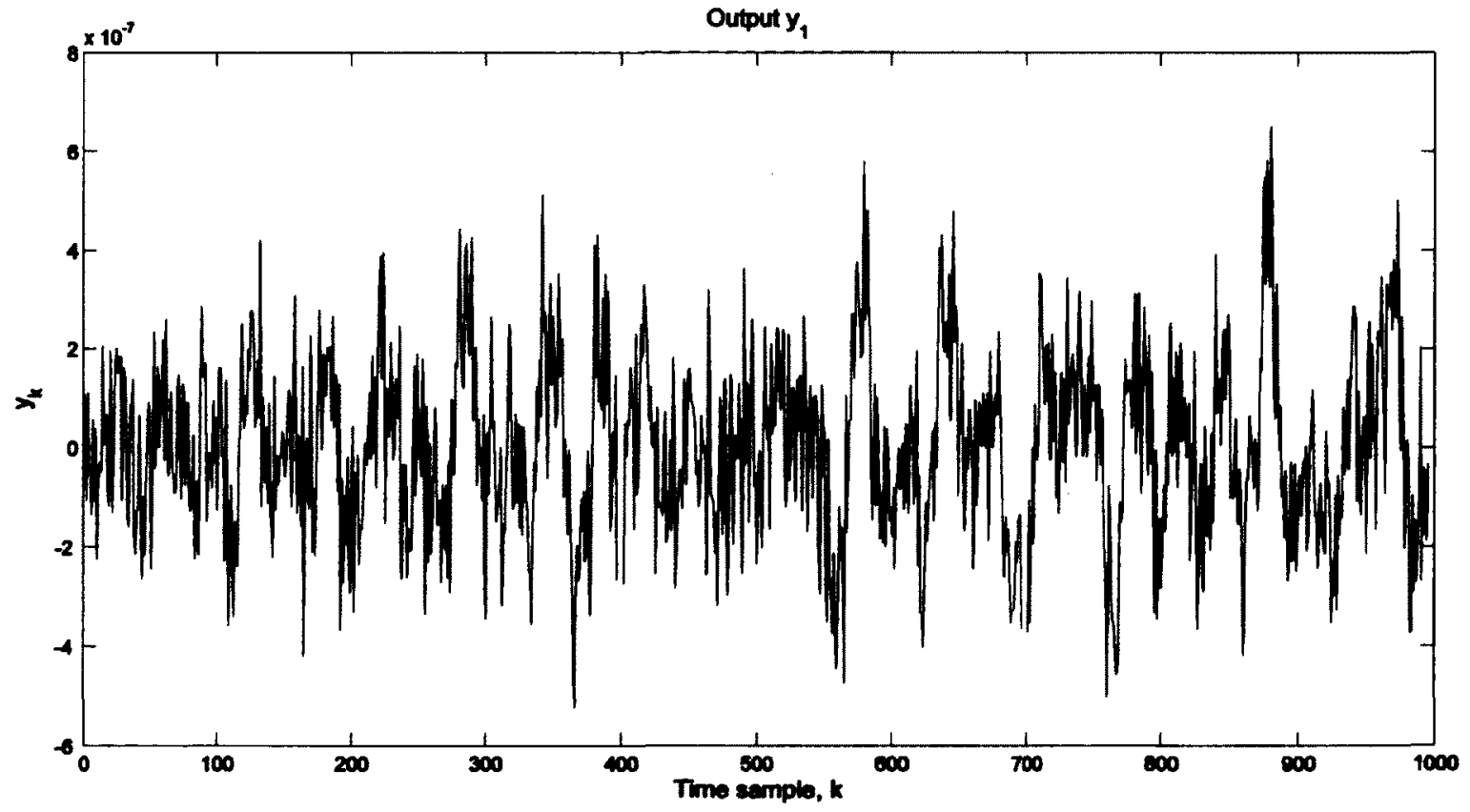

Figure 4-3: Part of the first output signal 


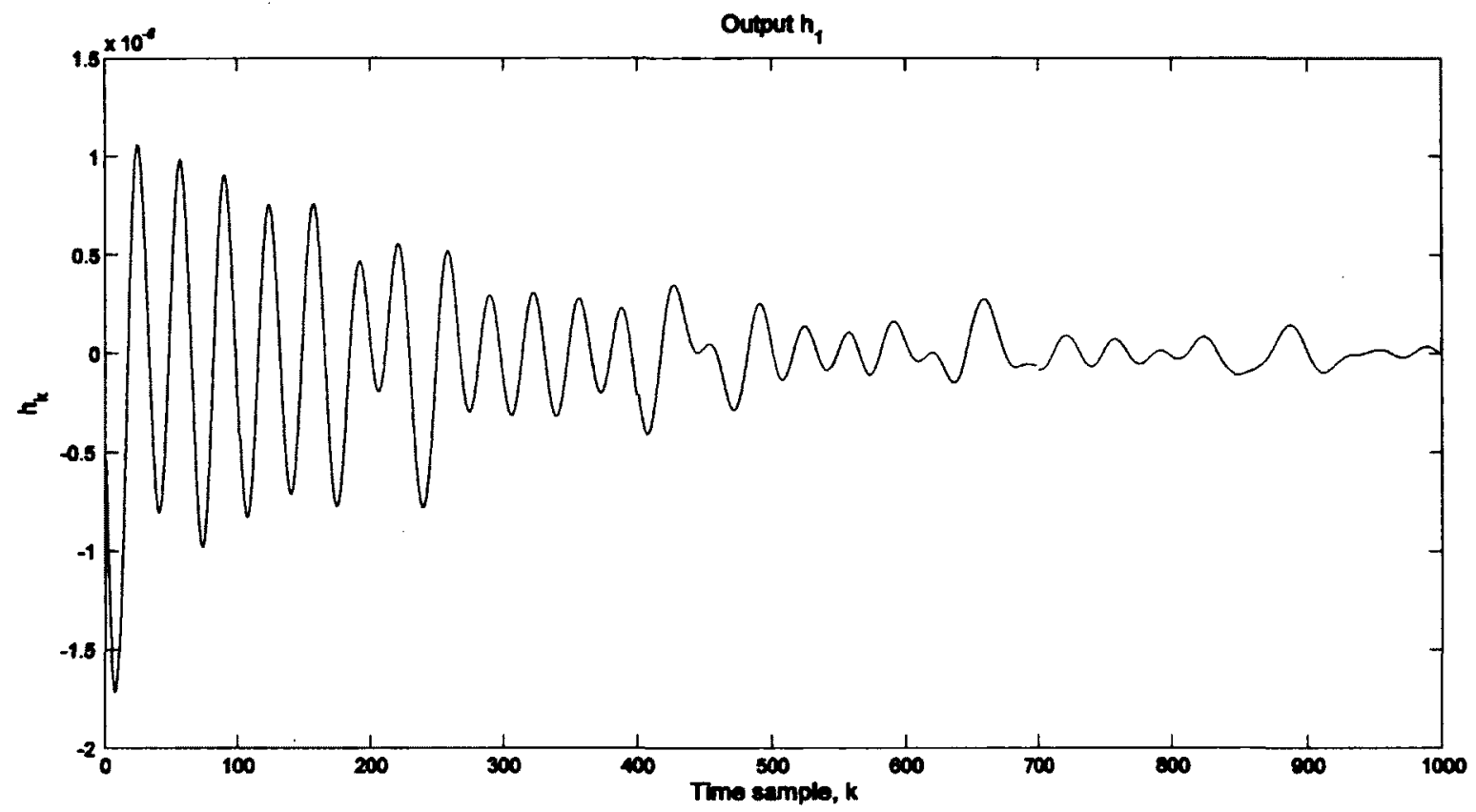

Figure 4-4: Part of the impulse response at $1^{\text {st }}$ DOF

Figure 4-5 shows the auto-power spectrum of first input and cross spectrum of first and second input. It can be observed that output forces are not entirely uncorrelated as cross spectrum is two order of magnitude smaller in magnitude in comparison to the auto-power spectrum.

The output data are filtered with a $12^{\text {th }}$ order Chebyshev type II low-pass filter with a cut off frequency of $5 \mathrm{~Hz}$ and stopband ripple of $60 \mathrm{db}$. Afterwards the data are resampled at $20 \mathrm{~Hz}$ to better estimate the low frequency modal parameter. This preprocessing also corresponds to the field practice where measurements are filtered with an analog low-pass filter to prevent aliasing. Finally noise is added to the simulated output measurements with $S N R=10 \mathrm{db}$. The modified first preprocessed output signal is shown in Figure 4-6. By comparing it with original signal 
(Figure 4-3) the effect of resampling of the data at a lower sampling rate is obvious. An estimated power spectrum of first (pre-processed) output location is shown in Figure 4-7. The spectrum does not go to zero as $f \rightarrow 0 \mathrm{~Hz}$ due to the presence of noise in the system. A typical correlation is shown in Figure 4-8.

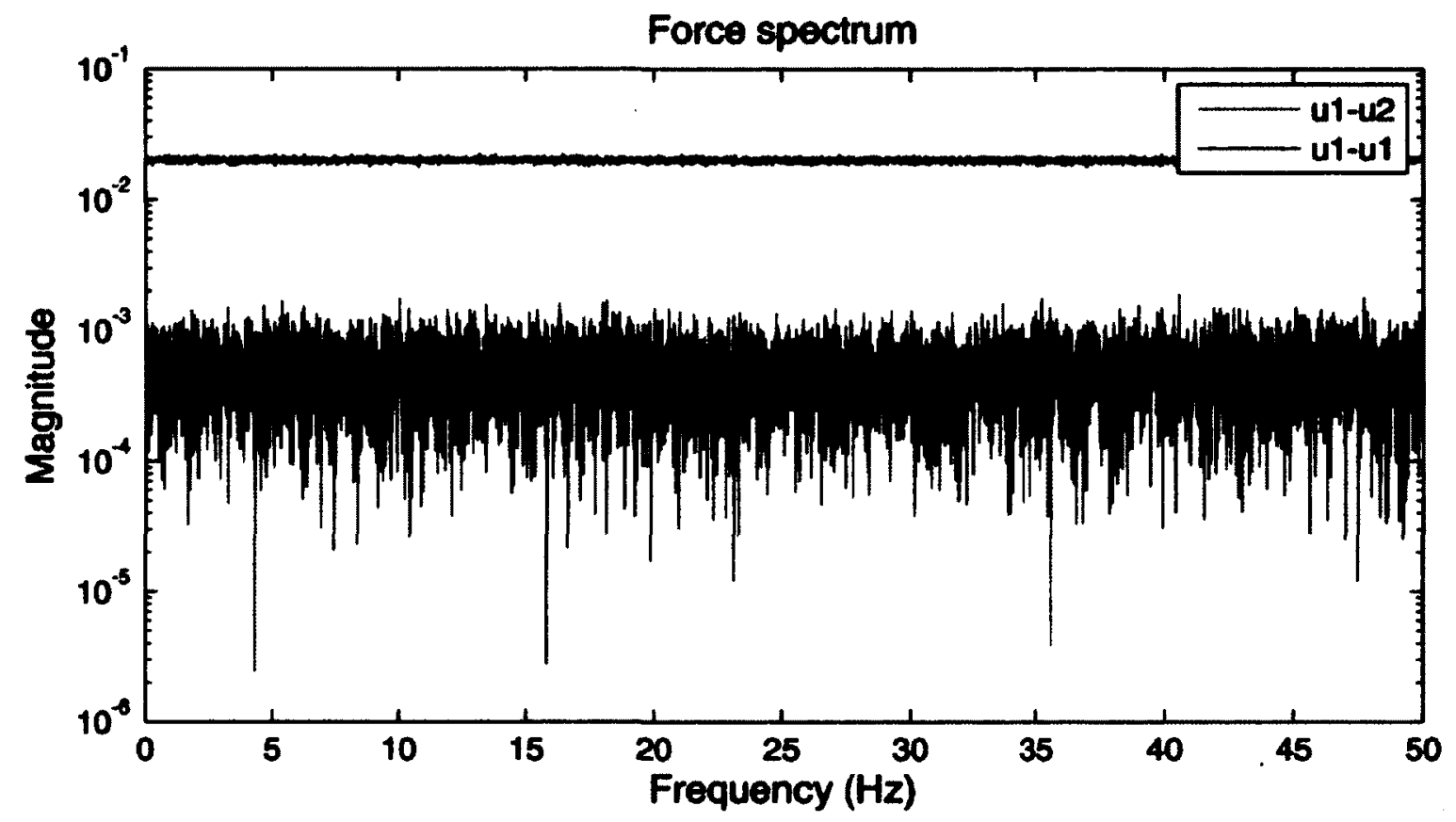

Figure 4-5: Input force spectra comparison

These pre-processed data are analyzed by different identification algorithms to evaluate their performance. The selected algorithms are ERA and SSI-COV in time domain, PolyMAX in frequency domain and FDD in spatial domain. For the PolyMAX method, the primary identification data is positive spectra whereas for the FDD method it is spectrum estimated by the Welch's periodogram method. For ERA and SSI-COV, the correlations for positive lag time with respect to the reference sensors are employed as identification data. 


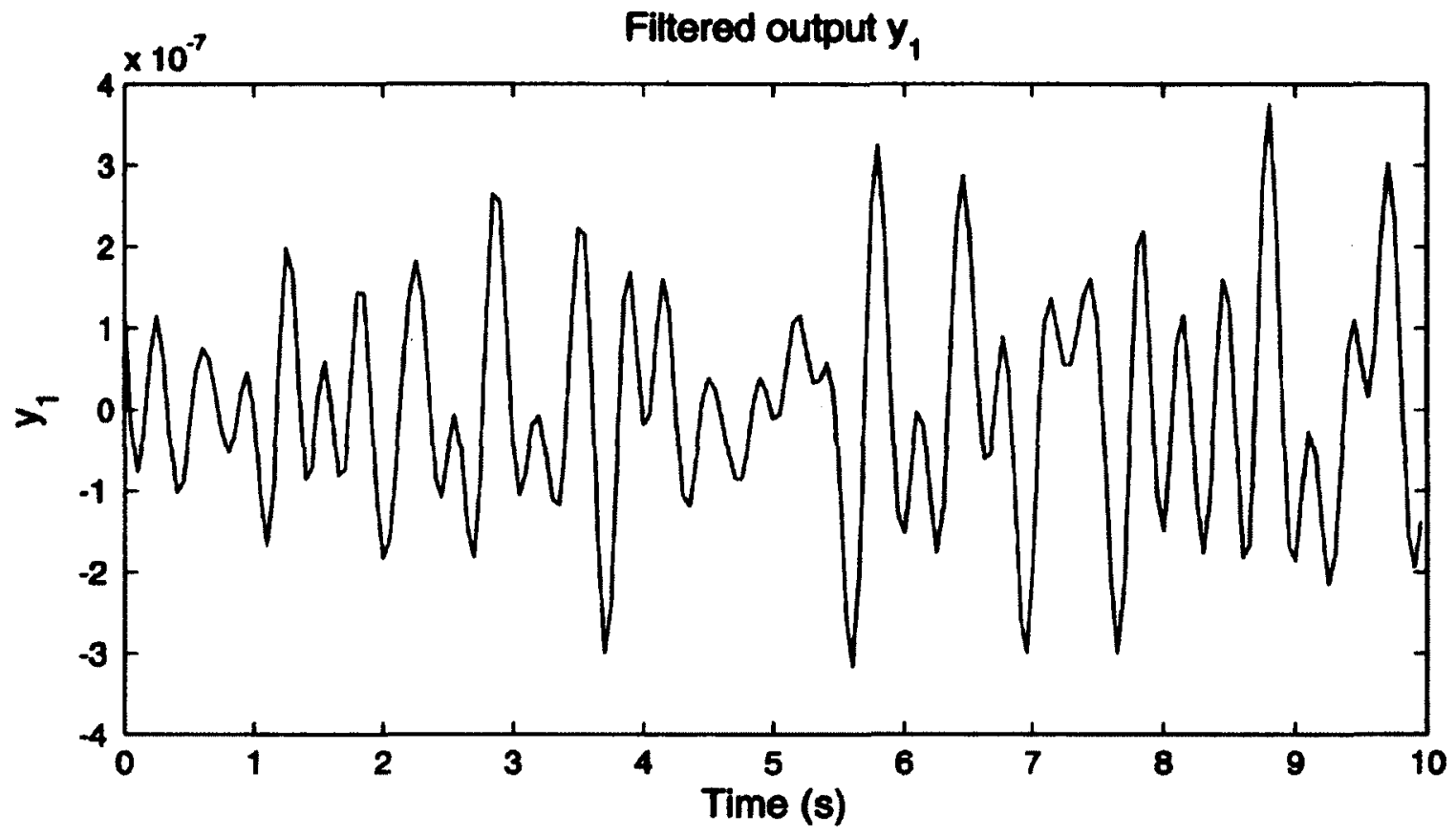

Figure 4-6: Part of the $1^{\text {st }}$ output signal (pre-processed)

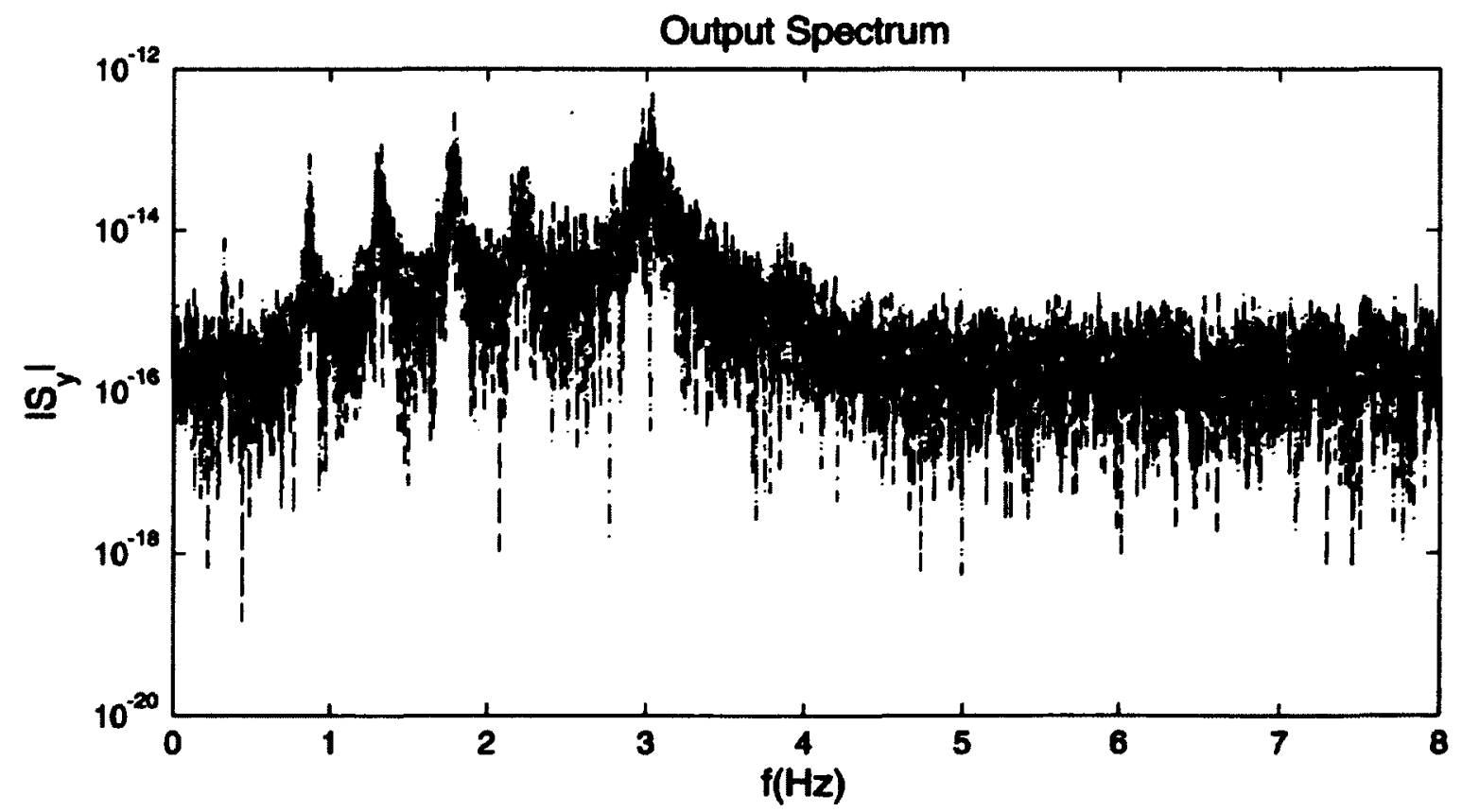

Figure 4-7: Estimated spectrum of $1^{\text {st }}$ output location 


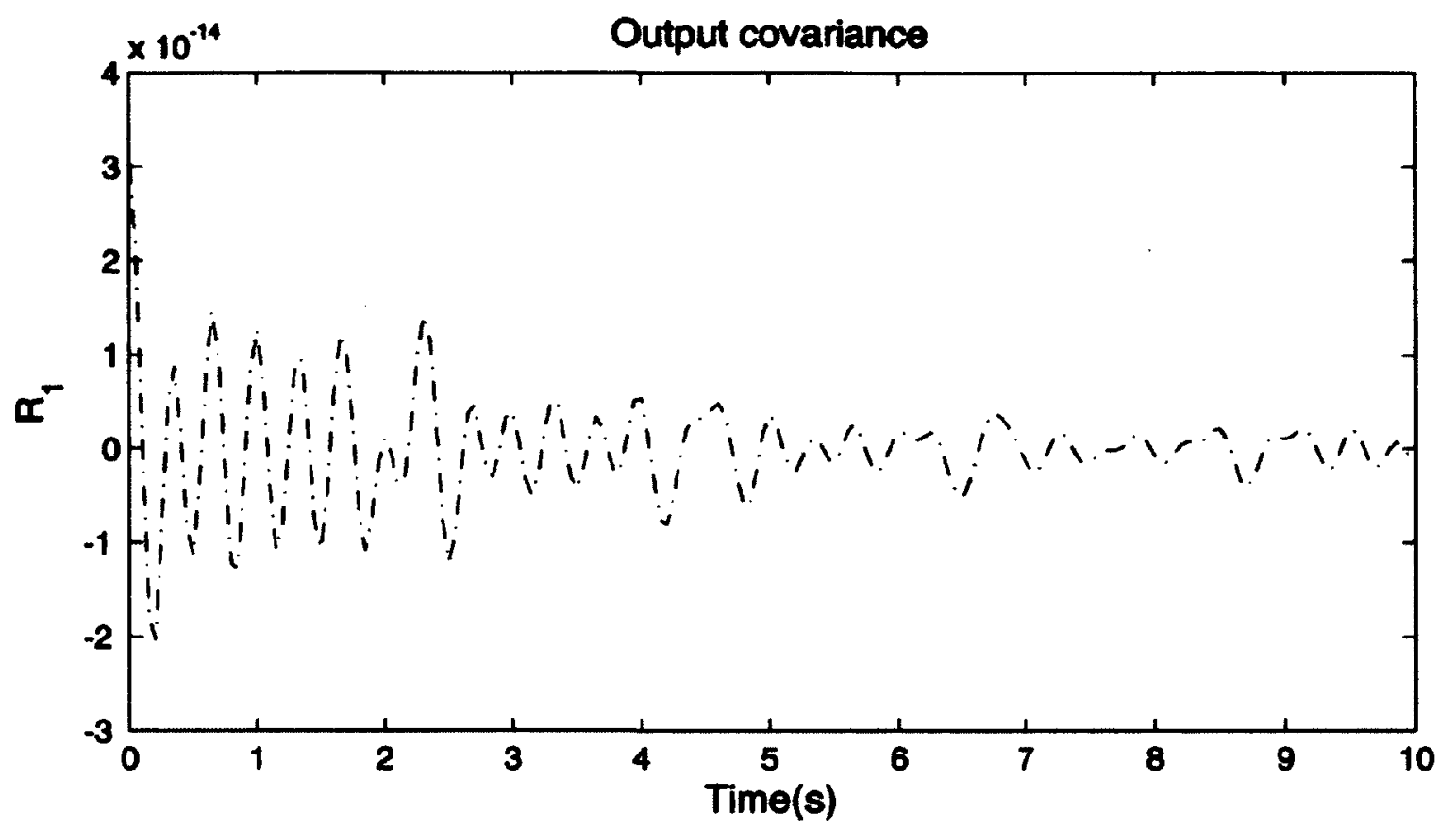

Figure 4-8: Estimated output covariance of $1^{\text {st }}$ output location

\section{Frequency Domain Decomposition (FDD) method}

FDD method is applied to the simulated example. The spectrum is estimated via the Welch's algorithm. The frequency resolution is $\Delta f=1 / T=.0098 \mathrm{~Hz}$ where $T$ is the measurement time of one segment $T=2048 \times .05 \mathrm{~s}$. The Singular Value Decomposition (SVD) is applied to the spectrum matrix. The obtained singular values as a function of frequency are plotted in Figure 4-9. Since, full spectrum matrix is used, there are eight singular values and four of them are presented in Figure 4-9. Only the first singular value is dominant indicating there is not mode multiplicity. So, theoretically it would be sufficient enough to estimate spectrum with respect to one reference sensor. The frequencies of the system can be selected 
as peak of the singular values (Figure 4-10). Around $3 \mathrm{~Hz}$ and $3.23 \mathrm{~Hz}$ there are spurious peaks that can be attributed to the typical erratic behavior of the nonparametric spectrum estimate. Modal assurance between estimated and true mode shapes is shown in Figure 4-11. It is noted that even though the FDD method identifies modal parameters quite well, the success of this process heavily depends on the expertise of the analyst. Damping estimate is performed in time domain assuming a SDOF around a peak. The identified frequency and damping are reported in Table 4-2. The detailed modal parameter estimation results are presented later at the end of this subsection.

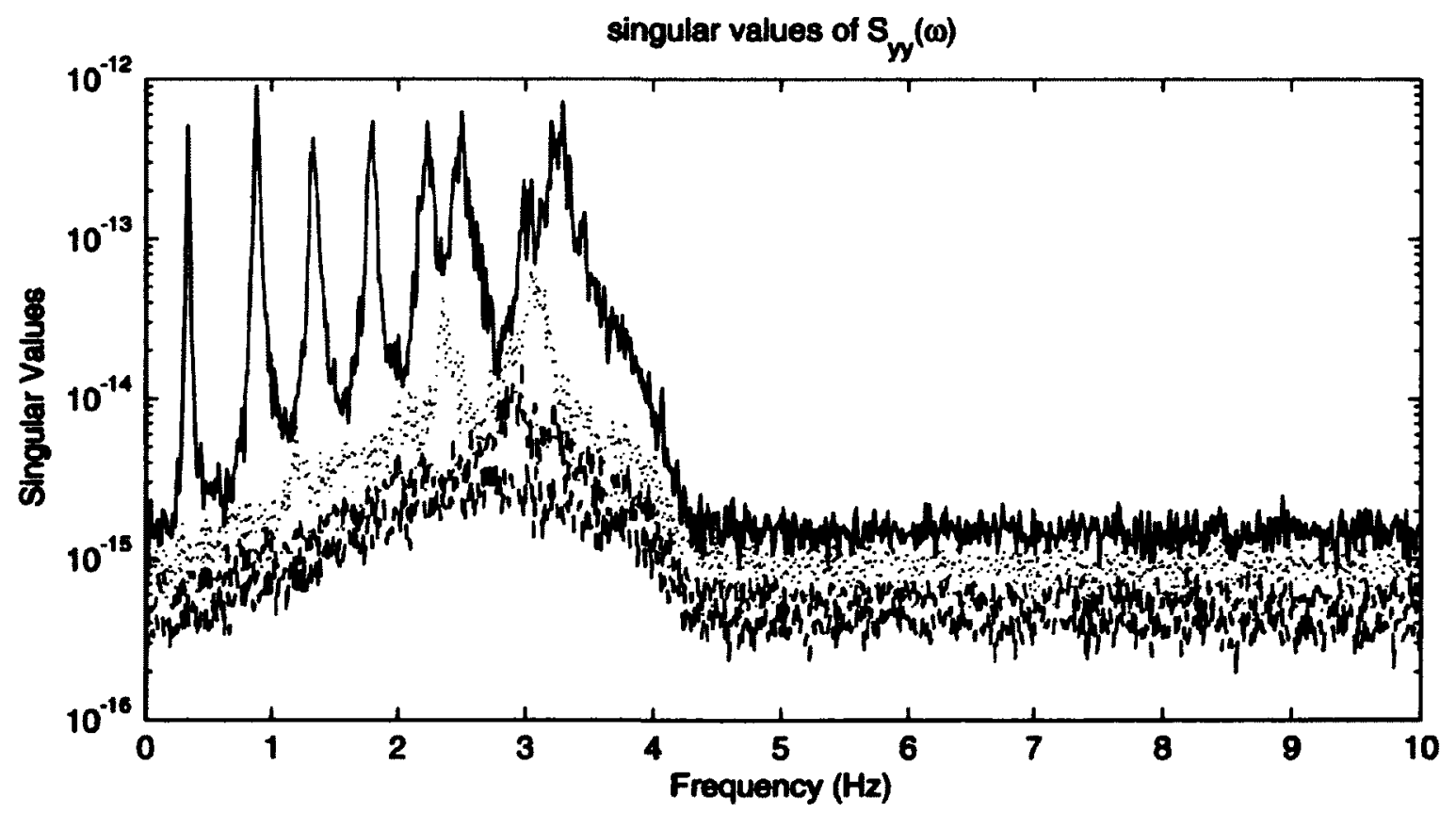

Figure 4-9: $1^{\text {st }}$ four singular values obtained by SVD of the full spectrum matrix 


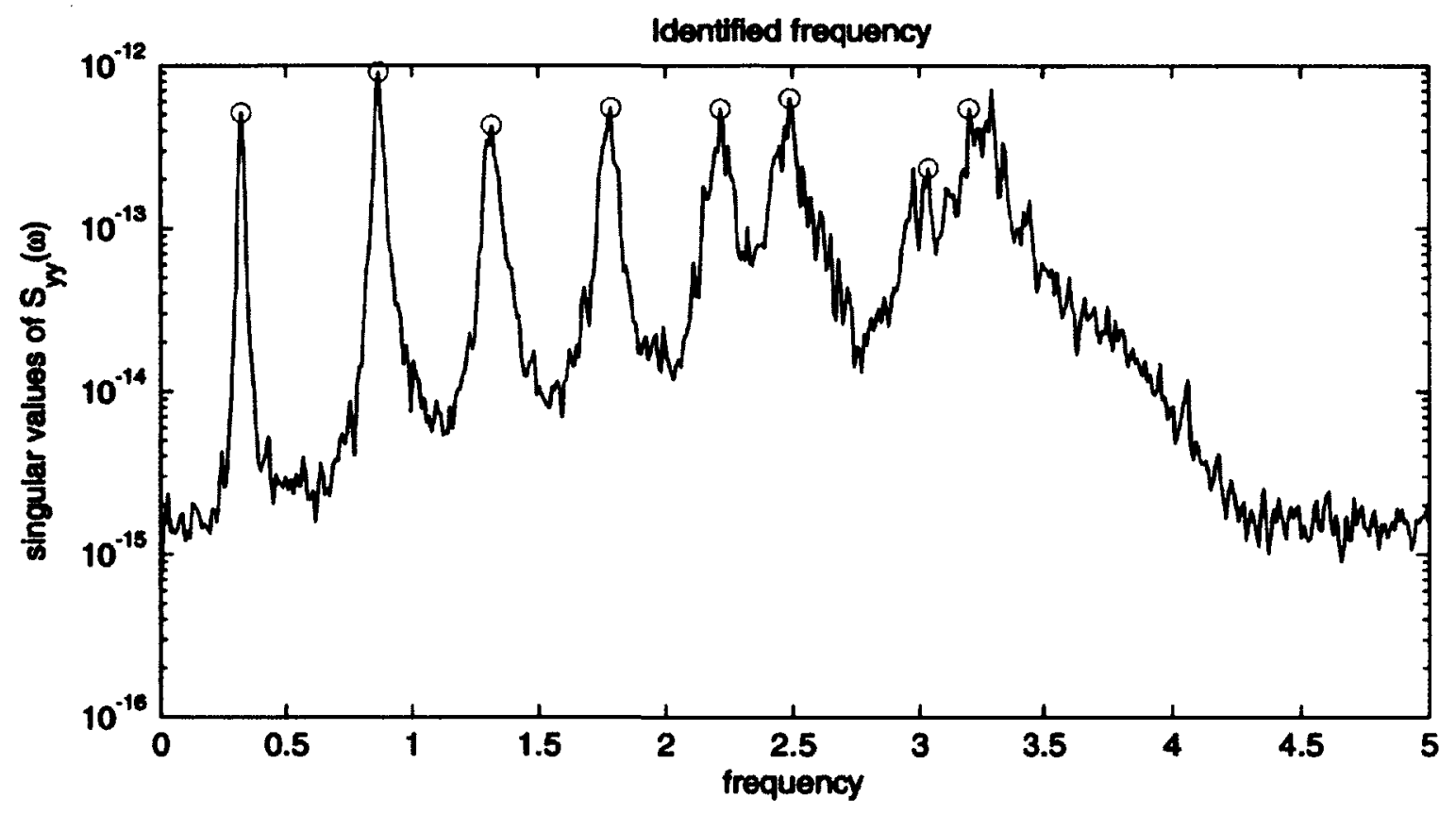

Figure 4-10: Identified frequencies from pick picking of singular value
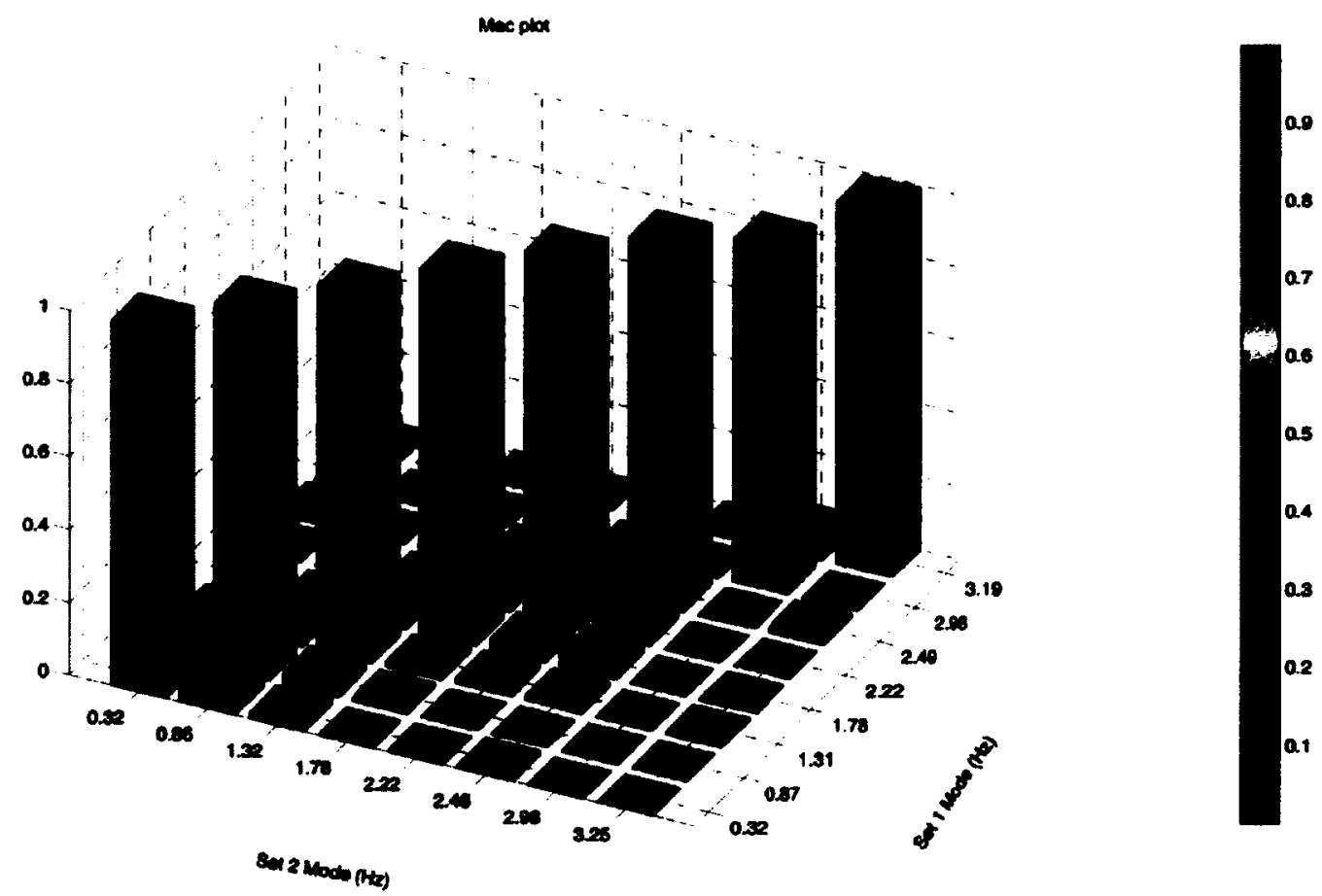

Figure 4-11: MAC plots for FDD algorithm (with the analytical mode shapes) 


\section{Eigensystem Realization Algorithm (ERA)}

By virtue of Natural Excitation Technique (NExT), data correlation of sensors with a reference sensor can be treated as free vibration response under the assumption of stationary process and thus make implementation of ERA in case of output only modal analysis. But proper implementation of the ERA method usually requires some knowledge of the structure as well as some experience with the algorithm, making it challenging to implement successfully for first-time users. For instance, Juang et al [22] suggested using a Hankel matrix whose number of columns is 20 times the number of frequencies and whose number of rows is $2-3$ times the number of columns. Another commonly used practice to select these dimensions is associated with the quality of the free responses [62]. Under this criterion, the Hankel matrix is built by making full use of the decaying signal provided that the signal to ratio is high. The identification of number of modes present in the data is usually performed by SVD of Hankel matrix (Equation 3-4). The truncation of singular values is also critical. Underestimate of th is value results in overlooking some vibrational modes while overestimation leads to computational modes. In this study, it is found that ERA persistently estimates 11 modes of the example system. The separation of the mathematical pole from physical pole is carried out based on the following rules:

- Modes with high damping ratio are discarded. A 5\% threshold is established for this simulated example.

- Repeated modes are eliminated based on the lowest energy content given by the 
singular values. Frequencies within $20 \%$ of each other and MAC value of 0.8 are used to identify such modes. However, in some instances, some modes are actually linear combination of other modes which are very hard to detect. Unfortunately, no action can be taken in such cases.

Correlations calculated with respect to sensor 2 and $\mathbf{5}$ are used as identification data. For the practical case of modal parameter identification, Hankel matrix is formed with 150 columns and 1200 rows that is equivalent to $11.3 \mathrm{~s}$ of free response data. The truncation of block Hankel matrix is carried out with 22 model order (Figure 4-12), so 11 modes are identified and then using the criteria mentioned above, the 8 vibrational modes are selected. The corresponding MAC plot is shown in Figure 4-13.

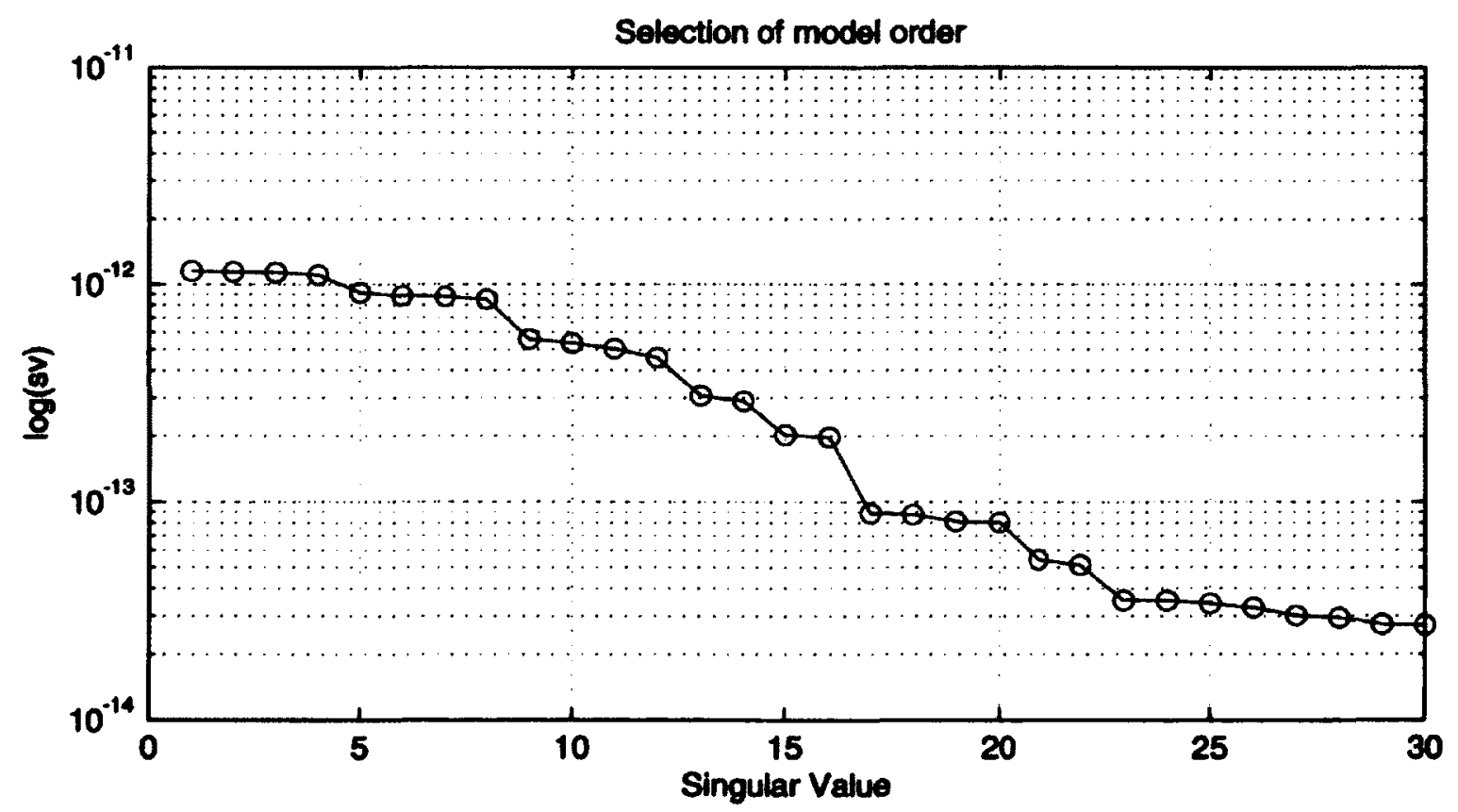

Figure 4-12: Singular values of the block Hankel matrix. The truncation is carried out 22 model order to account for some computational modes 


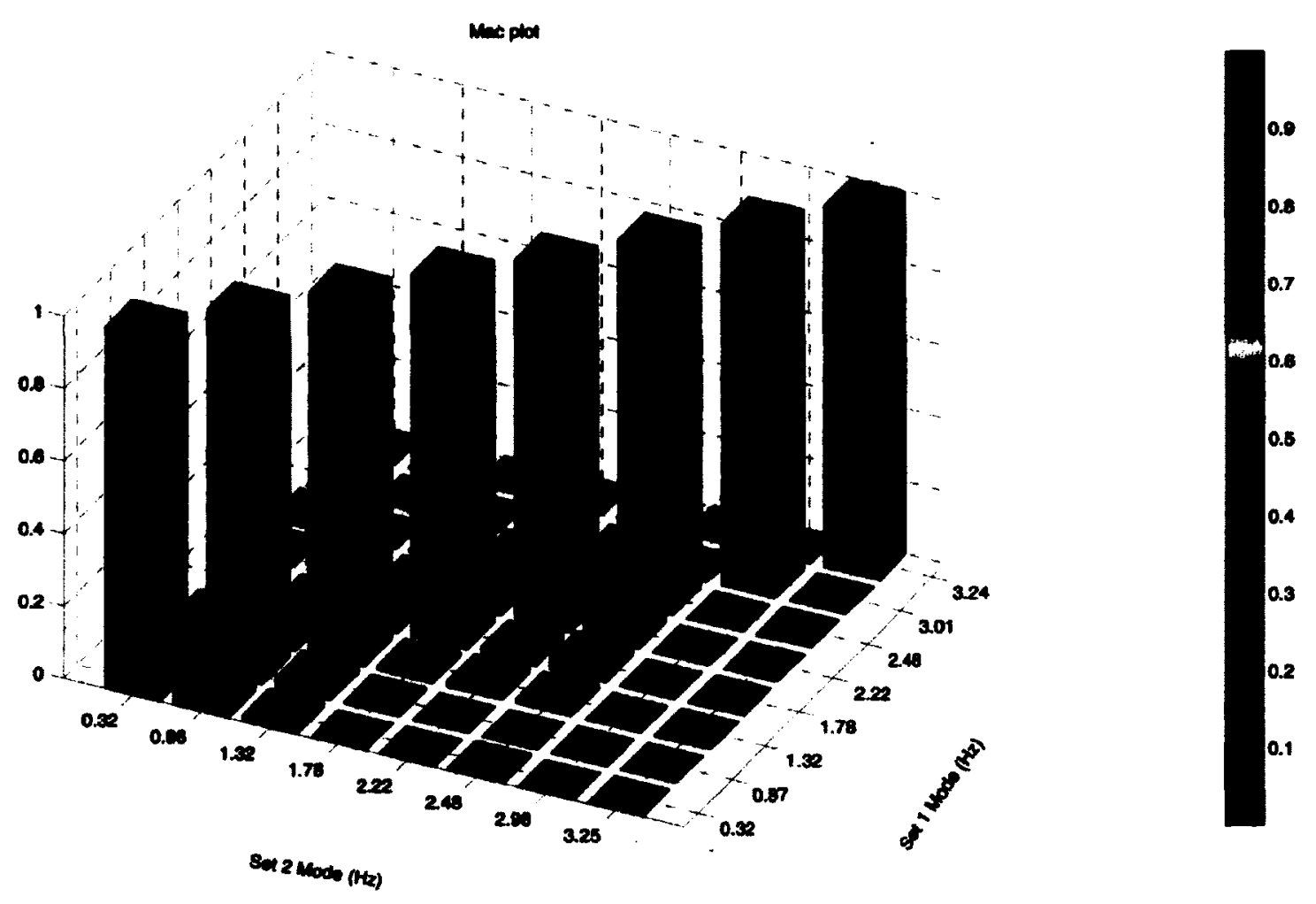

Figure 4-13: MAC plot between ERA identified modes and true modes

\section{Stochastic Identification (SSI) method}

SSI can be considered as the stochastic counterpart of the ERA method and based on the minimum realization theory. Parameters used in SSI method are as follows:

1) Maximum state-space model order $\left(n_{\max }\right)$ : 100 . This number corresponds to 50 structural vibration modes and is expected to exceed the true model order significantly. Even though this parameter does not have a direct impact on the analysis results, selecting a large number provides an easily interpretable stabilization diagram. 
2) Reference outputs. The accelerometers at locations 2 and 5 are used as reference sensor.

3) Number of time lags of the data correlation matrices ( $i$ parameter) is taken as 115. It satisfies the mathematical requirement $r \times i>n_{\max }$ as well as ensures enough information of lowest vibrational modes included in the analysis.

4) Stabilization of modes. To stabilize modal parameters, $0.5 \%$ limit is chosen for frequency, whereas for mode shape and damping the limits are $1 \%$ and $15 \%$ respectively. The elevated limit for damping stabilization can be attributed to the high uncertainty associated with damping estimation for OMA application. This issue is further explored at the end of this chapter.

Figure 4-14 shows the stabilization diagram for SSI method. It is evident that for low frequency content, stabilization diagram is somewhat not clear and needs an expert judgment to identify true poles. The identified modes are listed in Table 4-2 and the corresponding MAC plot is shown in Figure 4-16. As an accuracy indicator, the synthesized spectrum is compared with the measured spectrum (Figure 4-15). By looking at the synthesized spectrum it is obvious that damping estimate for less excited mode around $2.48 \mathrm{~Hz}$ is grossly underestimated by the SSI method. 


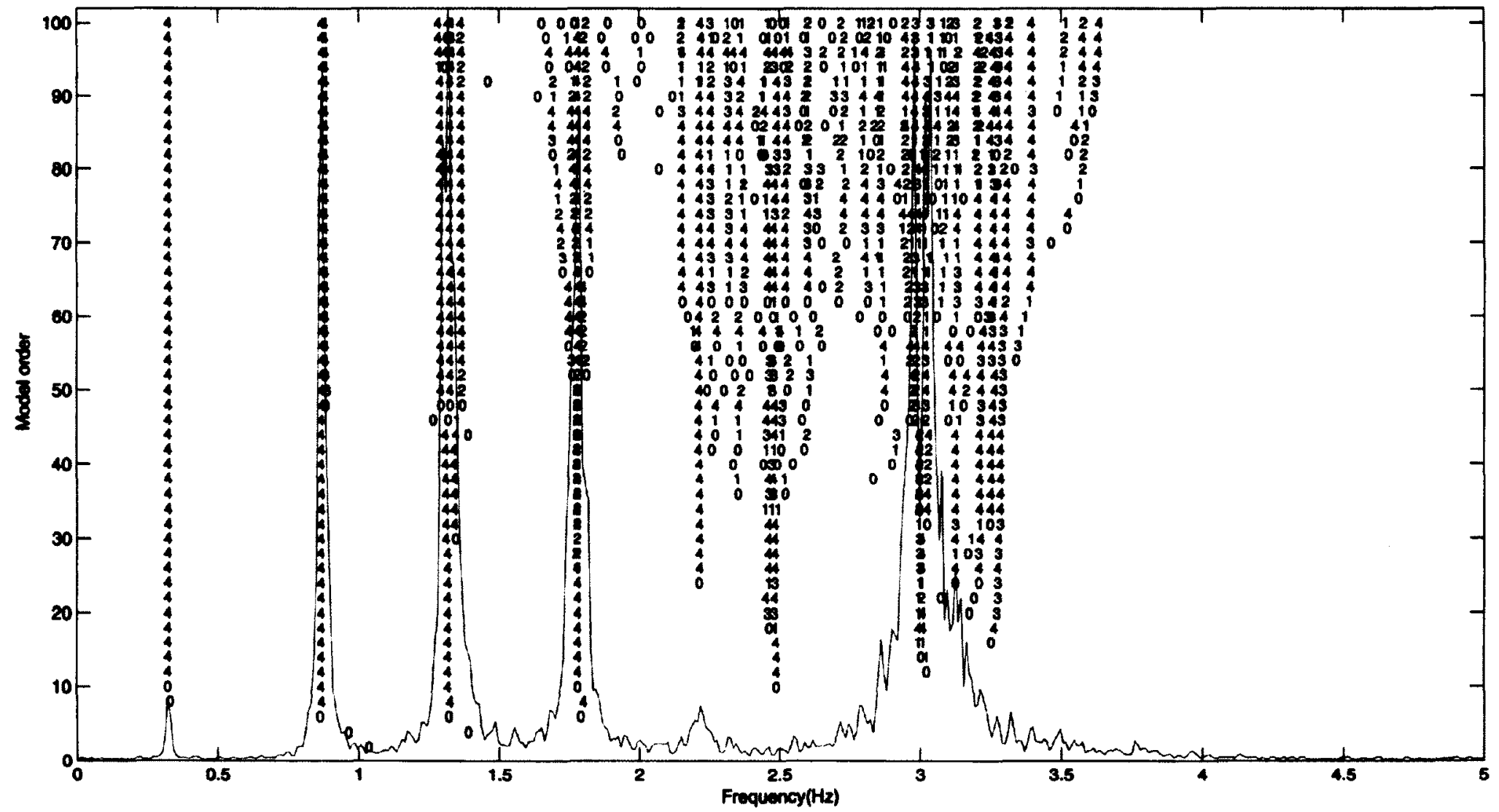

Figure 4-14: Stabilization diagram for SSI method. It is clear that for low frequency content around $2.5 \mathrm{~Hz}$ and $3.23 \mathrm{~Hz}$ stabilization diagram is somewhat misleading and needs an "expert" to distinguish between true and mathematical modes. Here " 4 " stands for stable pole, " 3 "stands for frequency and mode stable, " 2 " stands for frequency and damping stable and " 0 " stands for new frequency 


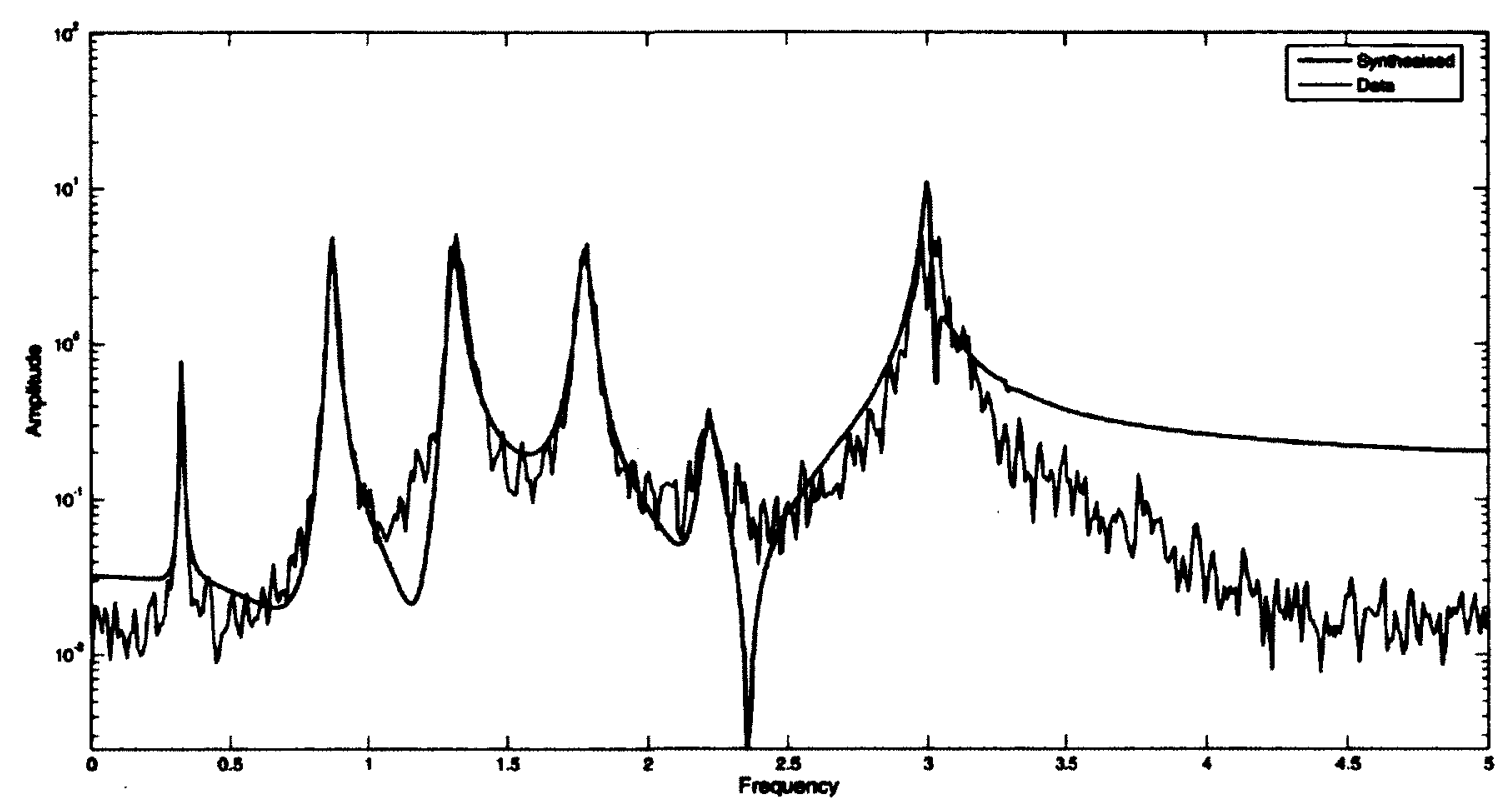

Figure 4-15: Synthesized auto spectra of reference sensor 2.
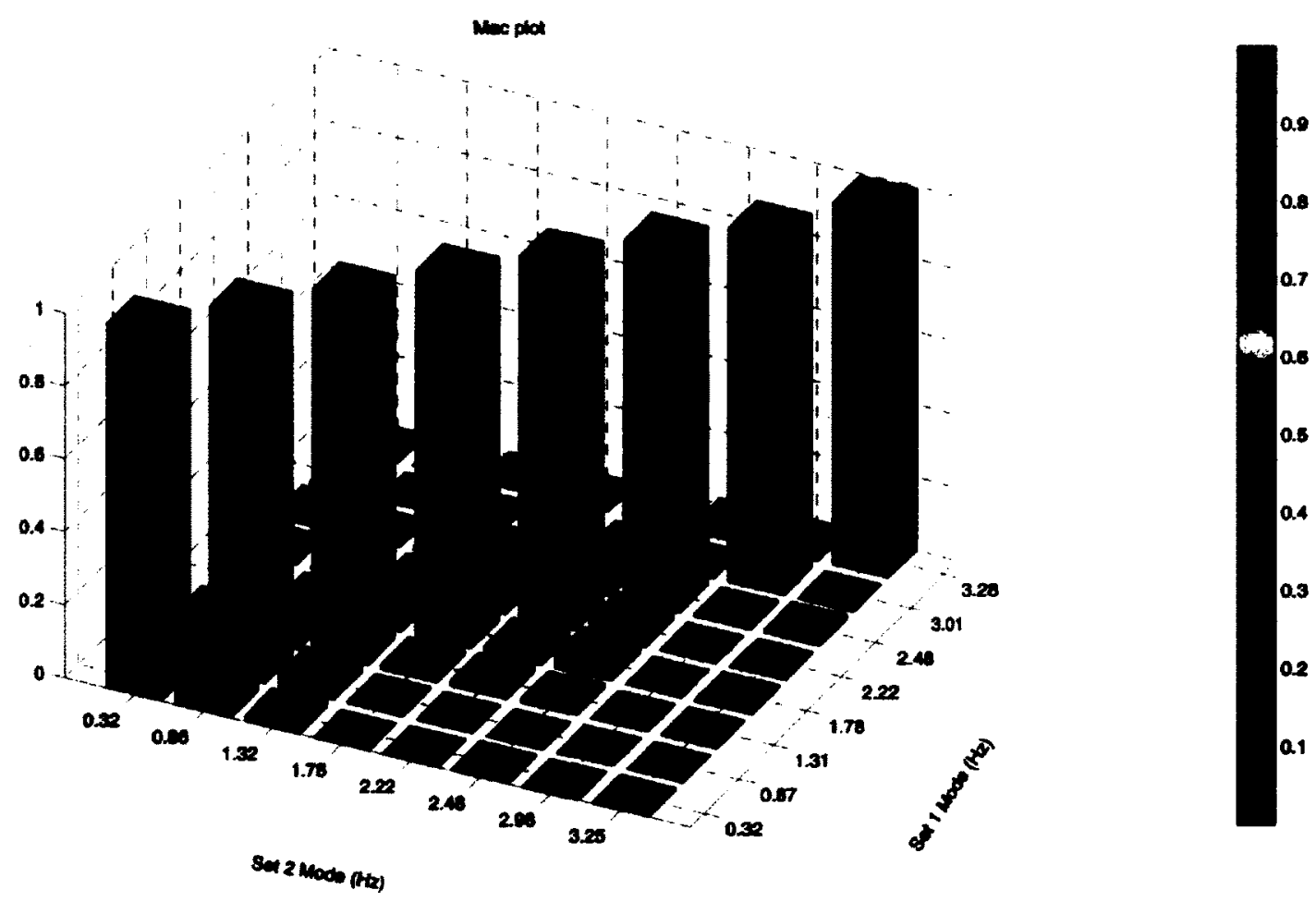

Figure 4-16: MAC plot between SSI and true modes 


\section{PolyMaX method}

This is the only frequency domain algorithm based on discrete-time model. One of the main attractive features of this algorithm is clear stabilization diagram. It has been shown that imposing proper parameter constraints in solving the denominator polynomial (Equation 3-64) in a least square sense place the unstable poles to fall outside the unit circle in the z-domain (Right hand side in Laplace domain). Here, the identification data, positive power spectrum, calculated from correlations with 250 lag time is carried out via 2048 points FFT with respect to reference sensors 2 and 5 . To reduce the leakage a $1 \%$ exponential window is applied prior to correlogram spectrum estimation. In generating the stabilization diagram, the same criteria of the SSI method is employed: $0.5 \%$ for frequency, $1 \%$ mode shape and $15 \%$ for damping. High speed FFT based method is used to estimate correlations as discussed in Section 3.5. The stables poles can be easily picked from clear stabilization diagram as shown in Figure 4-17. Again it is noted that modes with low frequency content needs higher model order to be stabilized. The identified modal parameters are presented in Table 4-2 together with the results from other methods. The corresponding MAC plot and synthesized spectra are shown in Figure 4-18 and Figure 4-19 respectively. Similar to the SSI method, it is clear that PolyMAX underestimates the damping for less excited modes (around $2.22 \mathrm{~Hz} \& 2.48 \mathrm{~Hz}$ ). 


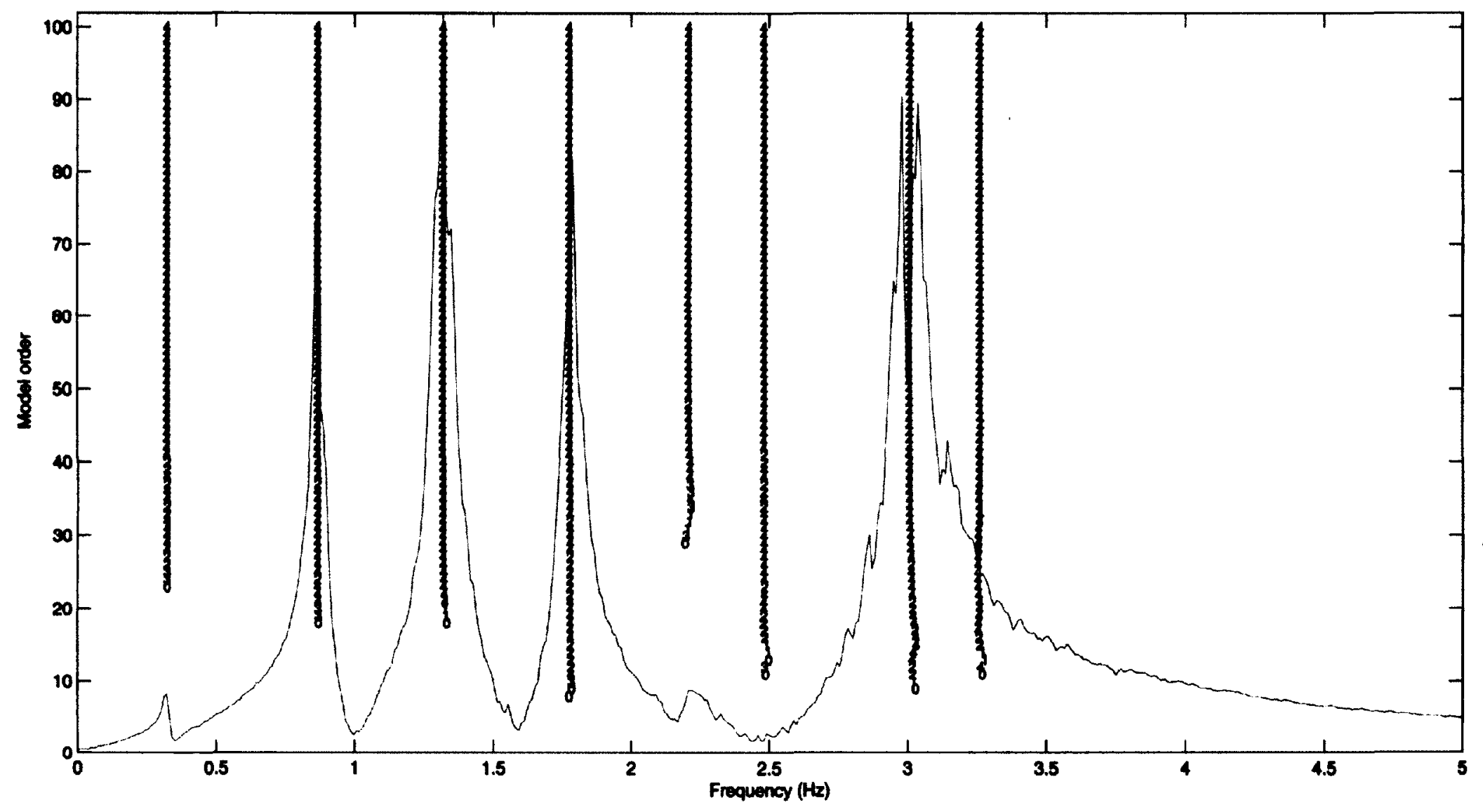

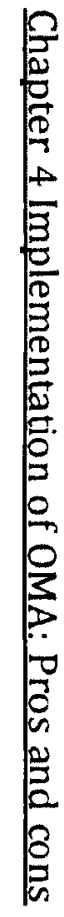

Figure 4-17: Clear stabilization diagram for PolyMAX method. Here "4" stands for stable pole, "3 "stands for frequency and mode stable, " 2 " stands for frequency and damping stable and " 0 " stands for new frequency 

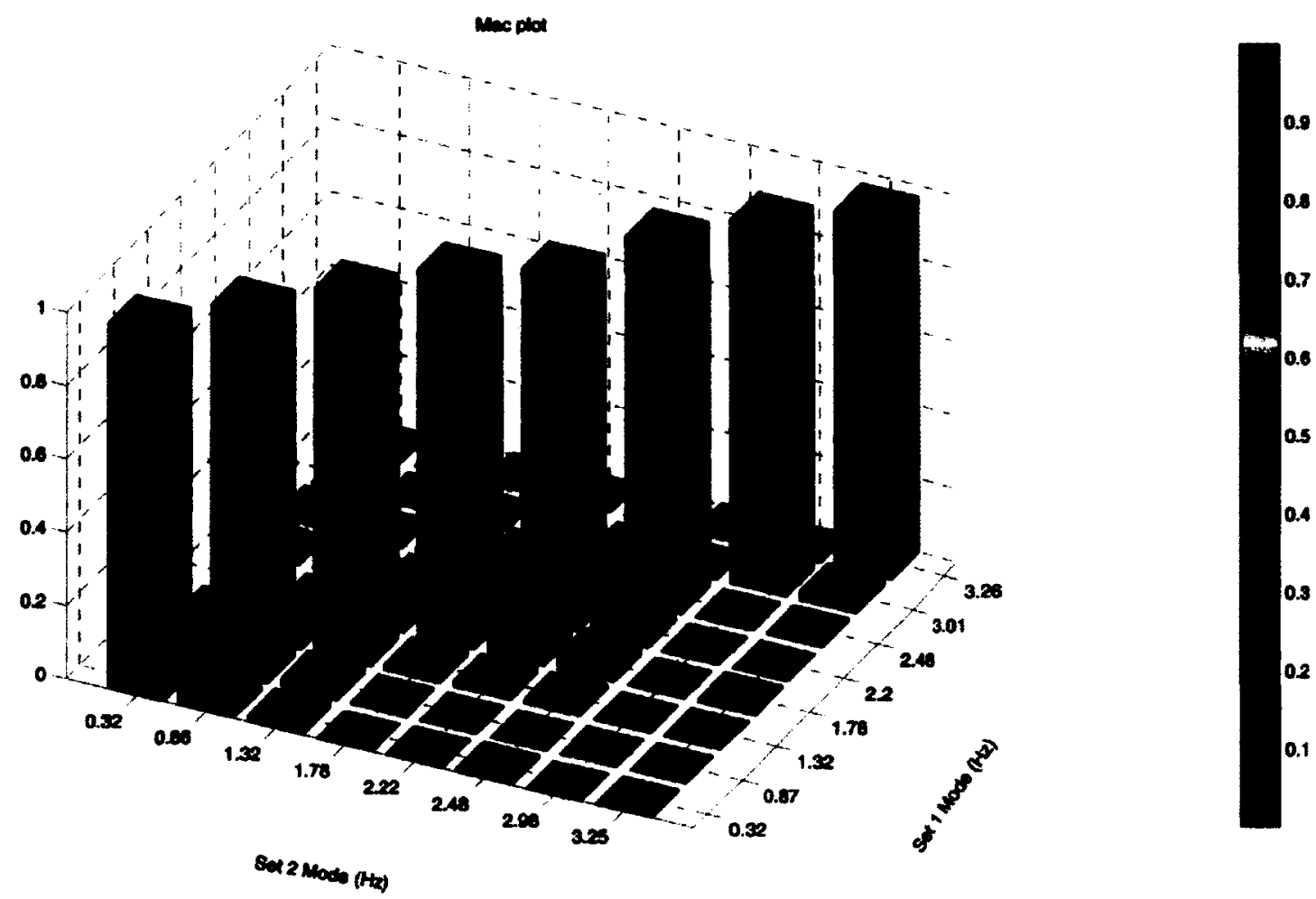

Figure 4-18: MAC plot between PolyMAX identified modes and theoretical modes

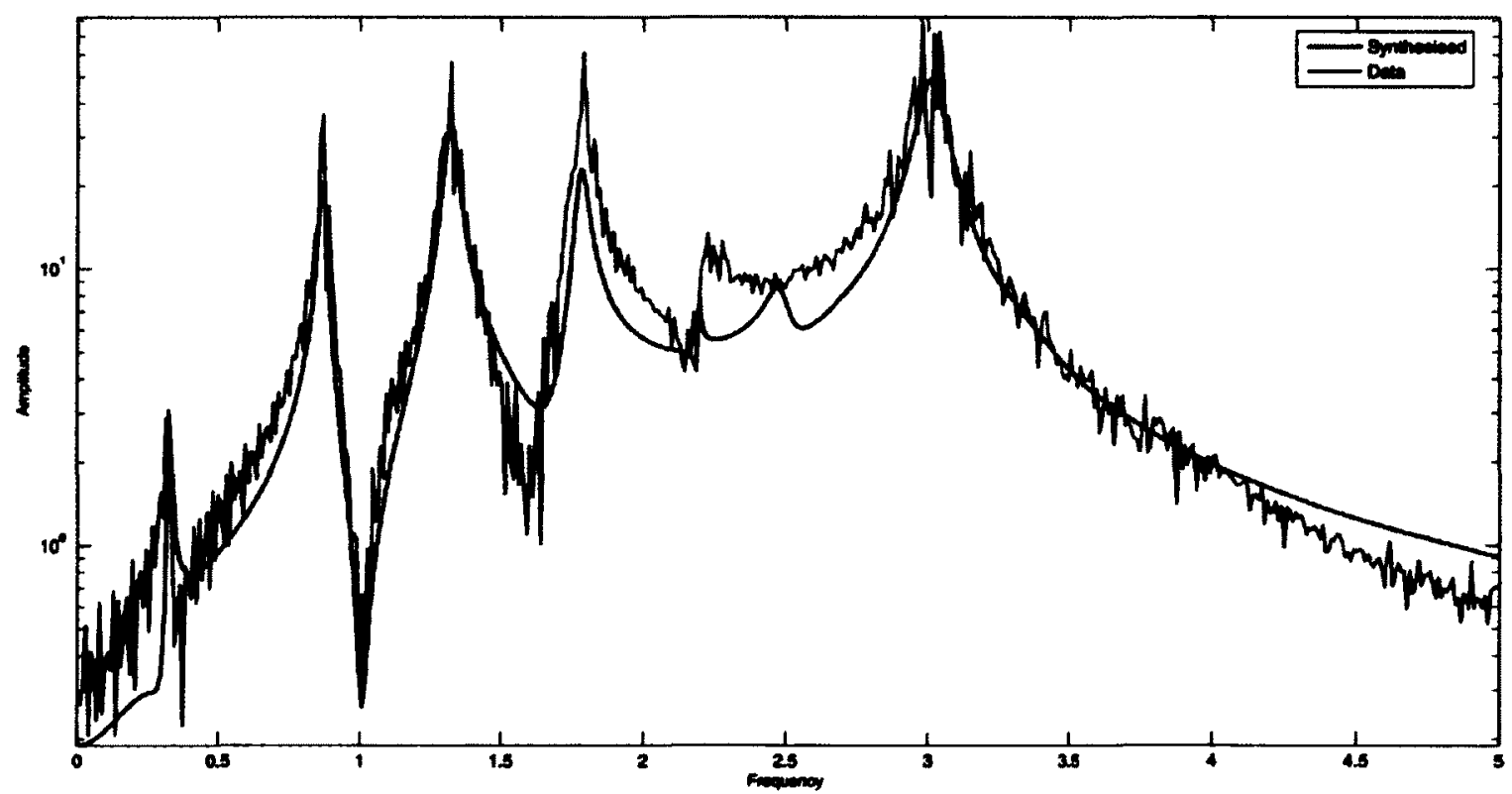

Figure 4-19: Synthesized auto spectra for reference sensor 2 
Table 4-2: Modal parameters estimated using different OMA algorithms

\begin{tabular}{|c|c|c|c|c|c|c|c|c|c|c|}
\hline \multirow[b]{2}{*}{$\#$} & \multicolumn{2}{|c|}{ Simulated system } & \multicolumn{2}{|c|}{ FDD } & \multicolumn{2}{|c|}{ ERA } & \multicolumn{2}{|c|}{ SSI } & \multicolumn{2}{|c|}{ PolyMAX } \\
\hline & $\begin{array}{l}\text { freq } \\
(\mathrm{Hz})\end{array}$ & $\begin{array}{l}\text { Damp } \\
(\%)\end{array}$ & $\begin{array}{l}\text { freq } \\
(\mathrm{Hz})\end{array}$ & $\begin{array}{l}\text { Damp } \\
\text { (\%) }\end{array}$ & $\begin{array}{l}\text { freq } \\
(\mathrm{Hz})\end{array}$ & $\begin{array}{l}\text { Damp } \\
(\%)\end{array}$ & $\begin{array}{l}\text { ireq } \\
(\mathrm{Hz})\end{array}$ & $\begin{array}{l}\text { Damp } \\
(\%)\end{array}$ & $\begin{array}{l}\text { freq } \\
(\mathrm{Hz})\end{array}$ & $\begin{array}{l}\text { Damp } \\
(\%)\end{array}$ \\
\hline 1 & 0.324 & 1.3000 & 0.323 & 2.2832 & 0.323 & 1.7984 & 0.323 & 1.4209 & 0.324 & 1.2935 \\
\hline 2 & 0.869 & 1.0392 & 0.866 & 1.2568 & 0.869 & 1.4294 & 0.866 & 1.3624 & 0.866 & 1.3077 \\
\hline 3 & 1.320 & 1.2386 & 1.309 & 1.9805 & 1.315 & 1.7904 & 1.309 & 1.6627 & 1.317 & 1.6239 \\
\hline 4 & 1.779 & 1.5118 & 1.781 & 1.5627 & 1.777 & 1.3913 & 1.777 & 1.3695 & 1.778 & 1.2458 \\
\hline 5 & 2.220 & 1.8000 & 2.223 & 1.8386 & 2.219 & 1.6708 & 2.216 & 1.4878 & 2.198 & 0.0570 \\
\hline 6 & 2.485 & 1.9798 & 2.490 & 2.0838 & 2.482 & 1.9240 & 2.483 & 0.4726 & 2.482 & 1.8455 \\
\hline 7 & 2.998 & 2.3368 & 3.037 & 3.0527 & 3.009 & 1.9992 & 3.005 & 0.5144 & 3.011 & 1.5470 \\
\hline 8 & 3.248 & 2.5132 & 3.193 & 1.3692 & 3.243 & 2.6255 & 3.278 & 0.1176 & 3.256 & 2.4650 \\
\hline
\end{tabular}

\subsection{Comparison of methods}

A discussion on the assumptions and limitations and practical implementation of a number of identification methods has been presented in the previous section. A more detailed and systematic comparison of the identification results in terms of the modal parameters is presented in this section. The comparison study of the performance of common system identification methods is carried is made by Monte Carlo simulation. It consists of the following steps:

- A white noise input is generated and applied to the simulated structure.

- White noise with SNR=10 db is added to each of the simulated output. 
- These "noisy" outputs are then analyzed by ERA, FDD, SSI and PolyMAX methods. Output 2 and 5 are used as references. This has the following effect on the primary data passed to the identification methods: the correlation between all channels and these reference sensors are computed (for ERA and SSI) and spectra between all channels and the references are computed (for FDD and PolyMAX). To ensure that same information is passed to every method; correlation computed up to $12.5 \mathrm{~s}$ are fed to all identification algorithms except the FDD method where all the data points are used to better estimate the spectra. For the case of PolyMAX method, to reduce leakage in spectra computation via the correlogram approach, a $1 \%$ exponential window is applied to the correlation estimate.

- All these steps are repeated for $\mathbf{1 0 0}$ different realizations of the input sequence and the measurement noise. Considerable effort is spent to minimize the user interaction during these 100 runs. For SSI and PolyMAX methods, the selection of poles relies upon an automatic interpretation of the stabilization diagram based on a rule based technique [63].

Among all these methods, the FDD method seems the most straight forward algorithm which doesn't need any user expertise. However it should be mentioned that sometimes picking the peak of SVD plot may not be enough. For the simple 8 DOF simulated example, in most of the runs, the inclusion of peaks that represent frequencies around $2.5 \mathrm{~Hz}, 3 \mathrm{~Hz}$ and $3.23 \mathrm{~Hz}$ are possible because of prior knowledge of the system matrix. Failing to do so would result in completely erroneous mode 
shape estimate. It is generally accepted that EMA algorithms does not perform well in the context of OMA applications. However, it turns out with the right choice of time segment (where the signal to noise ratio is high) and consequently using the Hankel matrix of proper size, the ERA method can estimate modal parameters as good as other OMA algorithms at least in terms of frequency and mode shape estimation. It is worth mentioning that ERA consistently estimates up to three computational modes although apriori knowledge of the model order plays a significant role in suitable truncation of Hankel matrix. This aspect, reasonable approximation of model order by looking at a "gap" of SVD plot, may be erroneous and misleading as for large, real structure there is no clear gap in SVD plot. In the next chapter, this issue is further explored with the Confederation Bridge monitoring data. On the other hand, both SSI and PolyMAX methods with the addition of stabilization diagram provide more accurate and clear interpretation of modal information in terms of model order and stabilized frequency. Apparently though, both the SSI and PolyMAX methods are not sufficiently reliable for identifying less excited modes i.e. frequency with low energy content. The SSI method fails to detect vibration modes of frequencies around $2.23 \mathrm{~Hz}, 2.48 \mathrm{~Hz}$ and $3.23 \mathrm{~Hz}$ in one third of the analysis cases and the selections are performed manually. Similarly, the PolyMAX method overlooks the frequency around $2.23 \mathrm{~Hz}$ for 20 runs. Nevertheless, it seems that positive power spectrum estimated from increased correlation lag time can partially overcome this difficulty as demonstrated in Figures 4-20 and 4-21. The adoption of a longer correlation lag time reduces the 
leakage error in estimating the spectra which improves the possibility that enough information of less excited modes is included in the analysis. The results of the simulation are presented in Figures 4-22, 4-23, 4-24 and summarized in Table 4-3. Figure 4-22 shows the eigenfrequency estimation results for 100 Monte-Carlo simulations. Bias and variance of the estimated frequency are tabulated in Table 4-3. The bias in frequency estimation is very small for all methods.

The damping estimates are presented in Figure 4-23 and Table 4-3. While error in the frequency estimate is very small, damping ratios are often off by $20-30 \%$ especially with the FDD method it is nearly 70\%. The SSI and PolyMAX methods perform better compared to the ERA and FDD methods particularly in terms of bias in damping estimate. In general, all the methods overestimate the damping ratios for the lower frequency modes. This concern is further discussed in Section 4.7. Figure 4-24 and Table 4-3 represent the modes shape results in MAC. Consideration of system information in peak picking of the SVD plot is the reason why FDD performs quite well. As mentioned previously, the SSI method has difficulty for picking the low energy frequency and it is reflected in MAC number for modes 5, 6 and 8. The results show that that ERA method is comparable with SSI in mode shape estimation, though the beforehand knowledge of model order somewhat helps to decide on the truncation level of Hankel matrix. Quite surprisingly, both variance and bias of mode shape estimate by the PolyMAX method are higher than other methods especially for mode 5. Even though it is small, the standard deviation of PolyMAX estimate is three times higher than other methods. 


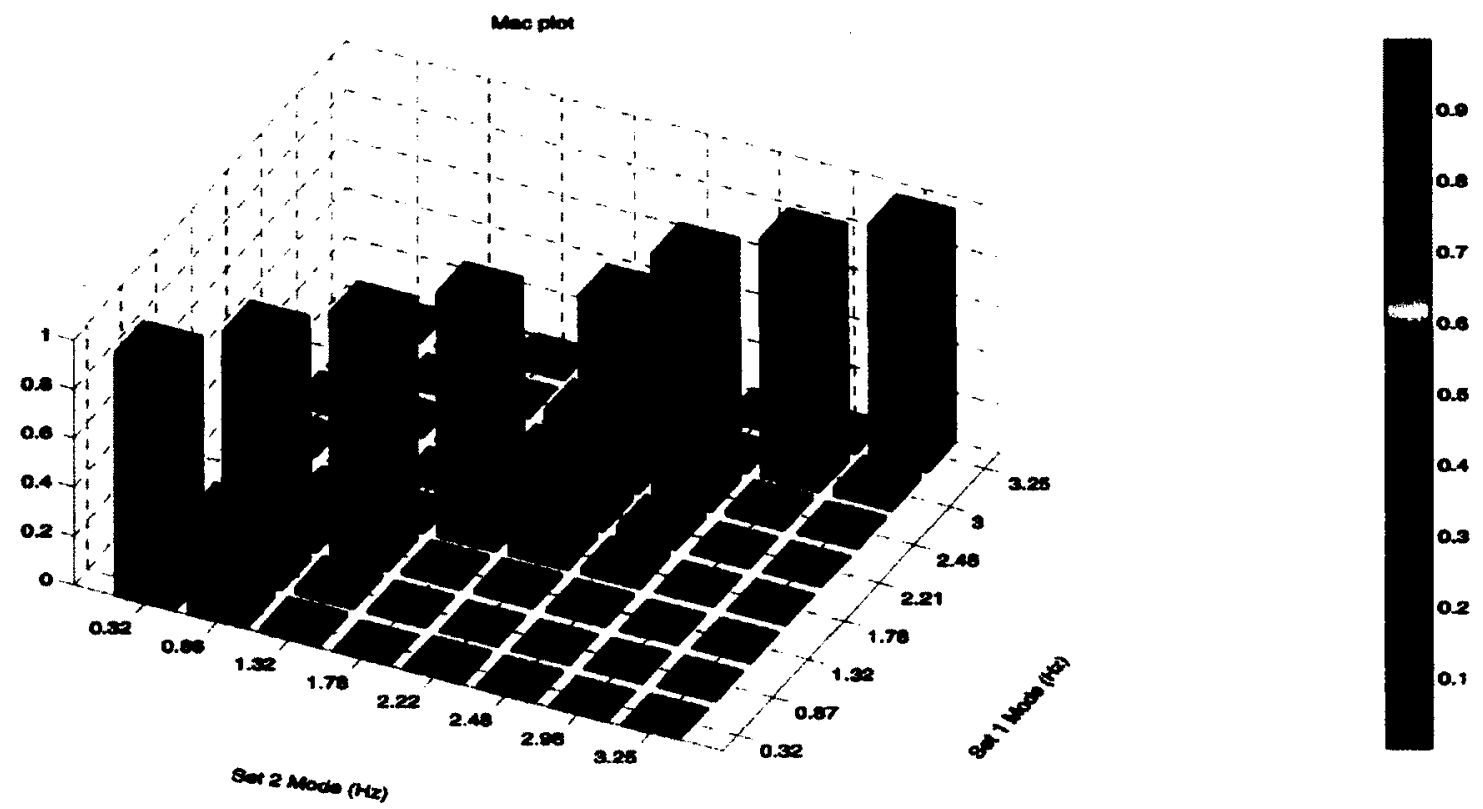

Figure 4-20: MAC plot for one run (PolyMAX). Frequency at $2.21 \mathrm{~Hz}$ is not captured at all. Spectra are calculated with 200 lag time \& 1\% exponential window.

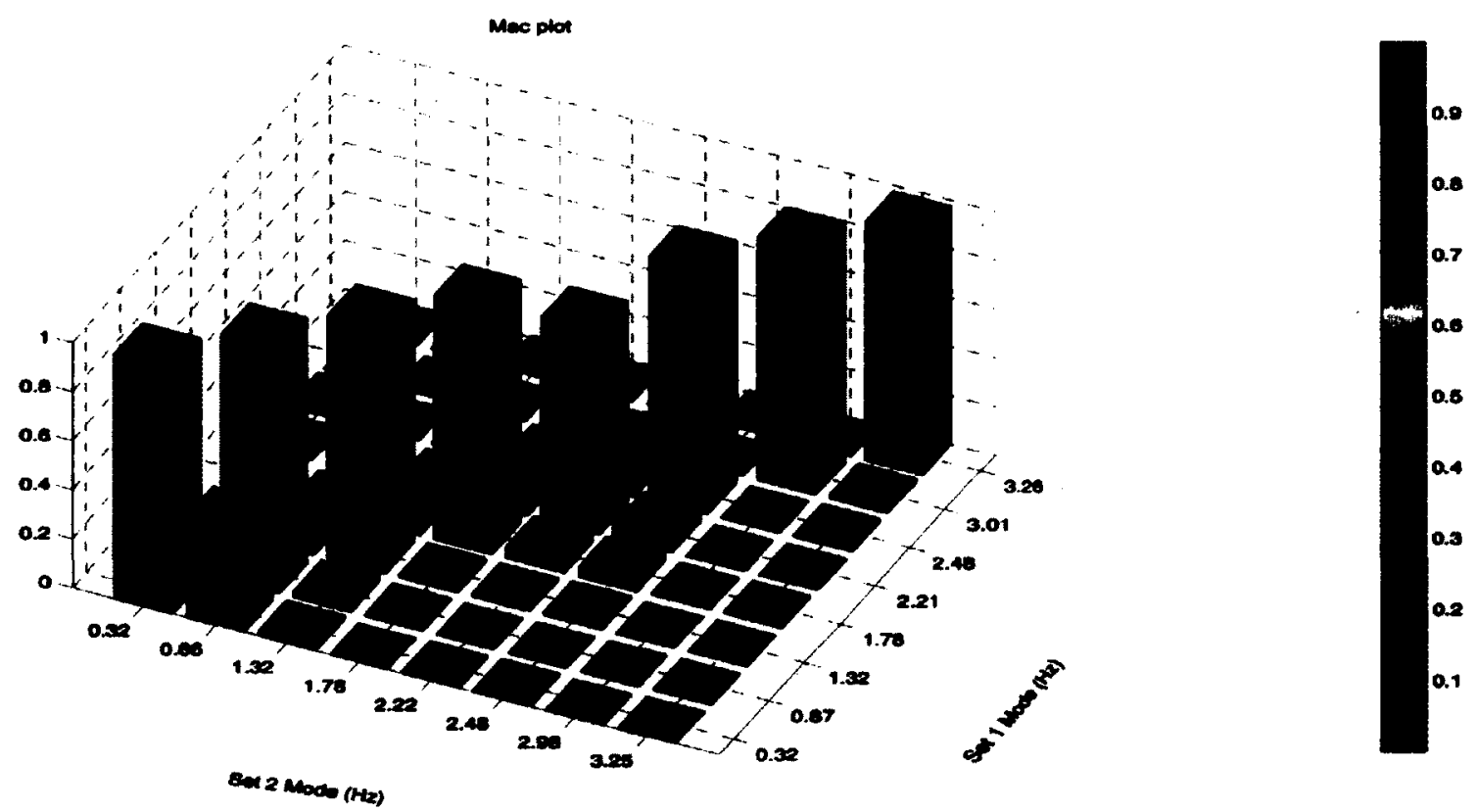

Figure 4-21: MAC plot for same run (PolyMAX). Frequency at $2.21 \mathrm{~Hz}$ is now captured properly. Spectra are calculated with 1200 lag time \& 1\% exponential window. 

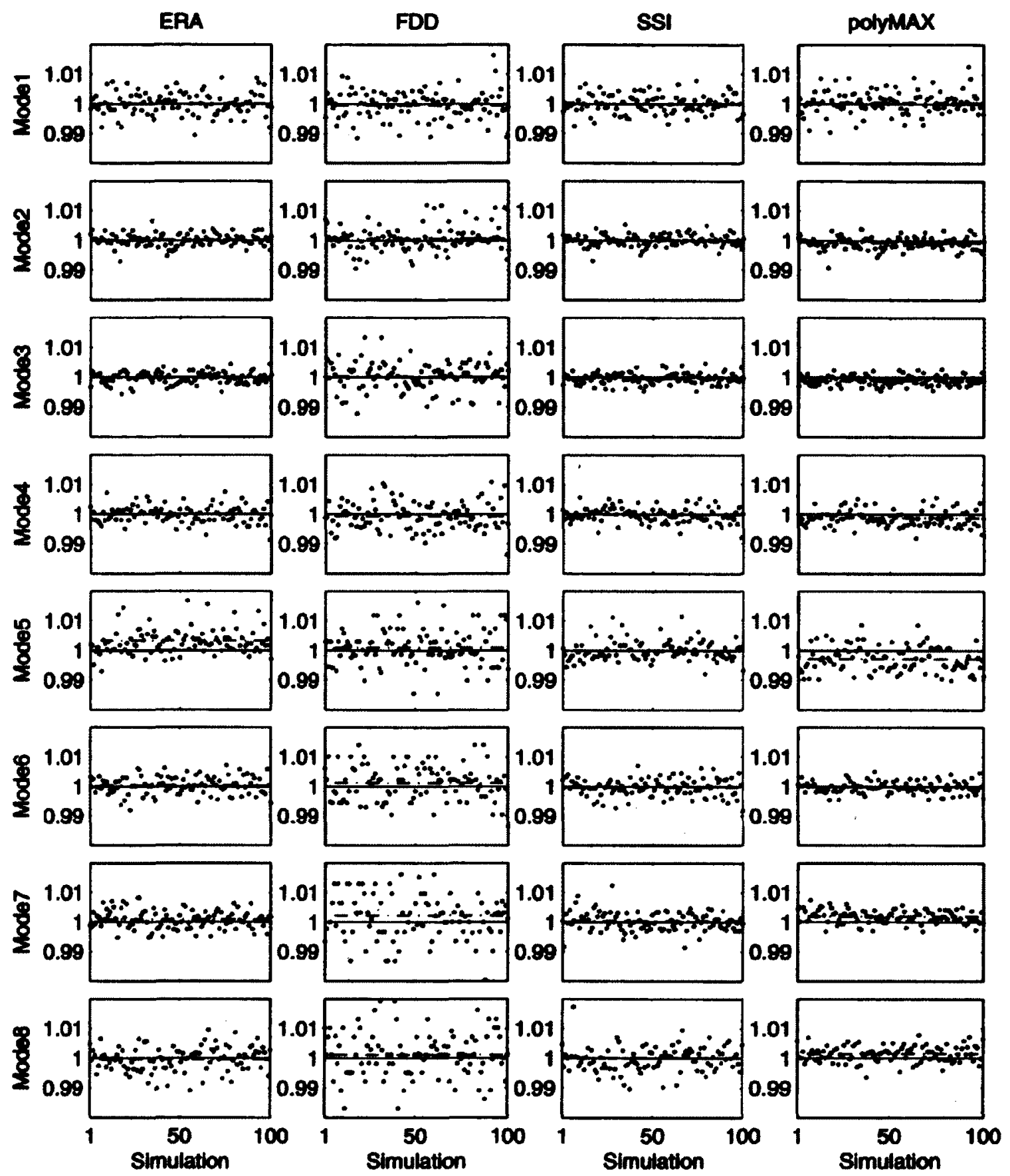

Figure 4-22: Normalized frequency estimate from 100 Monte-Carlo simulations. A value of 1 indicates a perfect estimate. The relative frequencies are shown as dots and the scatter gives an idea about the variance. The average estimate is also shown as a dashed line. 

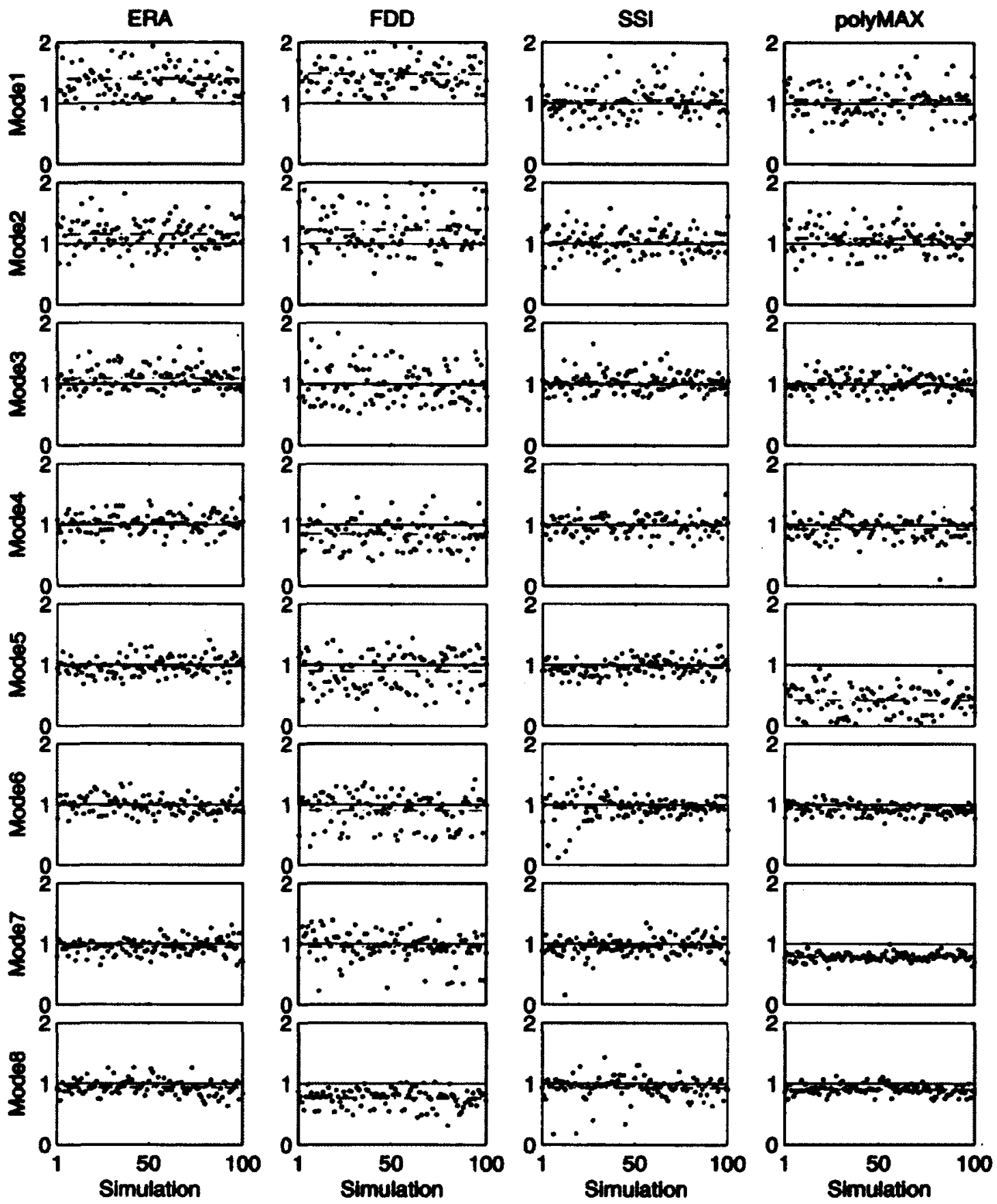

Figure 4-23: Normalized damping estimate from 100 Monte-Carlo simulations. A value of 1 indicates a perfect estimate. The relative damping is shown as dots and the scatter gives an idea about the variance. All the methods are seriously affected by high bias. The average estimate is also shown as a dashed line. 


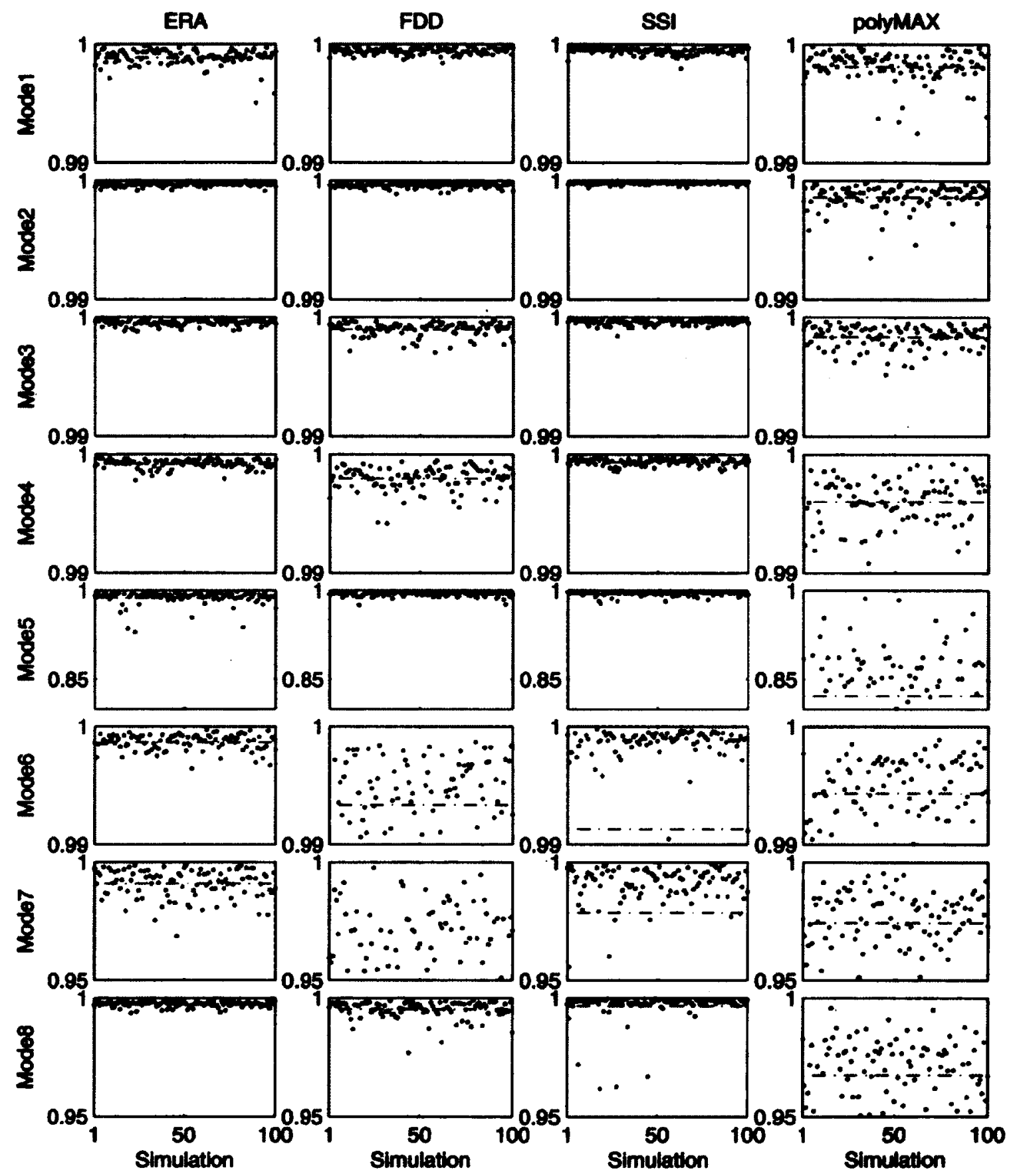

Figure 4-24: Mode shape estimation results from 100 Monte-Carlo simulations. The MAC between the estimation and the theoretical mode shapes are presented as dots. It seems that among all the methods, the effect of bias and variance error is higher in PolyMAX method. 
Table 4-3: Modal parameter estimation results from 100 Monte-Carlo simulations. The relative bias and relative std. deviations are given.

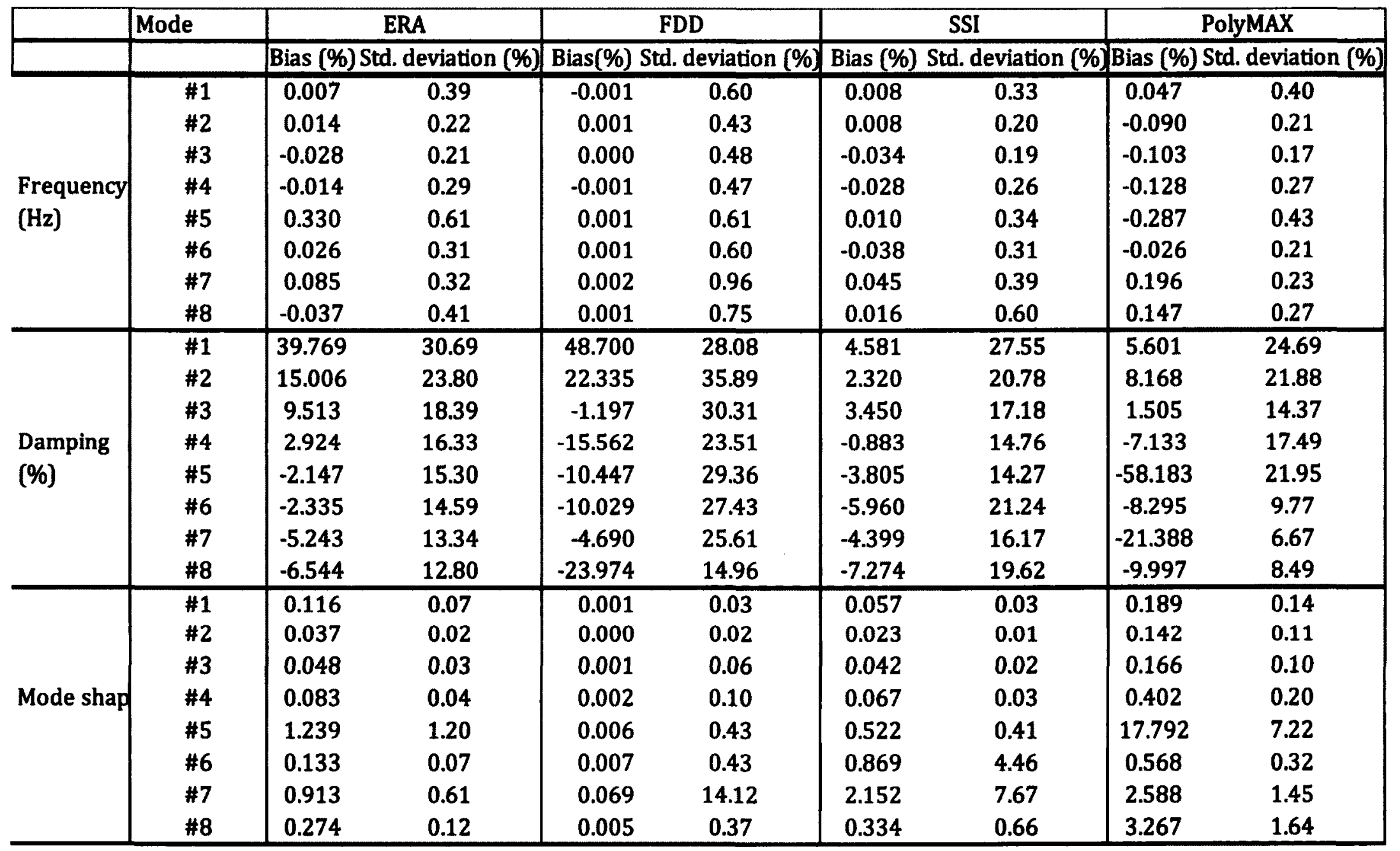




\subsection{Effect of violating OMA assumptions}

The development of OMA heavily relies upon the stationary uncorrelated white input noise assumption. It is also assumed that the loading is spatially complete i.e. all DOFs are excited. But, in reality, these assumptions may be questionable. In this section, the effects of violating these assumptions are studied with the same 8-DOF simulated example.

\section{Case 1: Presence of harmonics}

The 8-DOF is excited with white noise along with a $1 \mathrm{~Hz}$ sinusoid at DOF 3 . It is clear from the SSI stabilization diagram; the frequency component at $1 \mathrm{~Hz}$ appears as spurious poles of the state matrix $A$ and cannot be separated from eigenfrequency of the system as shown in Figure 4-25. The same conclusion can be reached with the PolyMAX stabilization diagram shown in Figure 4-26. Similarly, the FDD method detects a peak in SVD plot at $1 \mathrm{~Hz}$ (Figure 4-27) where ERA method identifies $1 \mathrm{~Hz}$ as a stable frequency and ignores other low energy content at mode $2.23 \mathrm{~Hz}$. In brief, no method is effective in the presence of harmonics. 


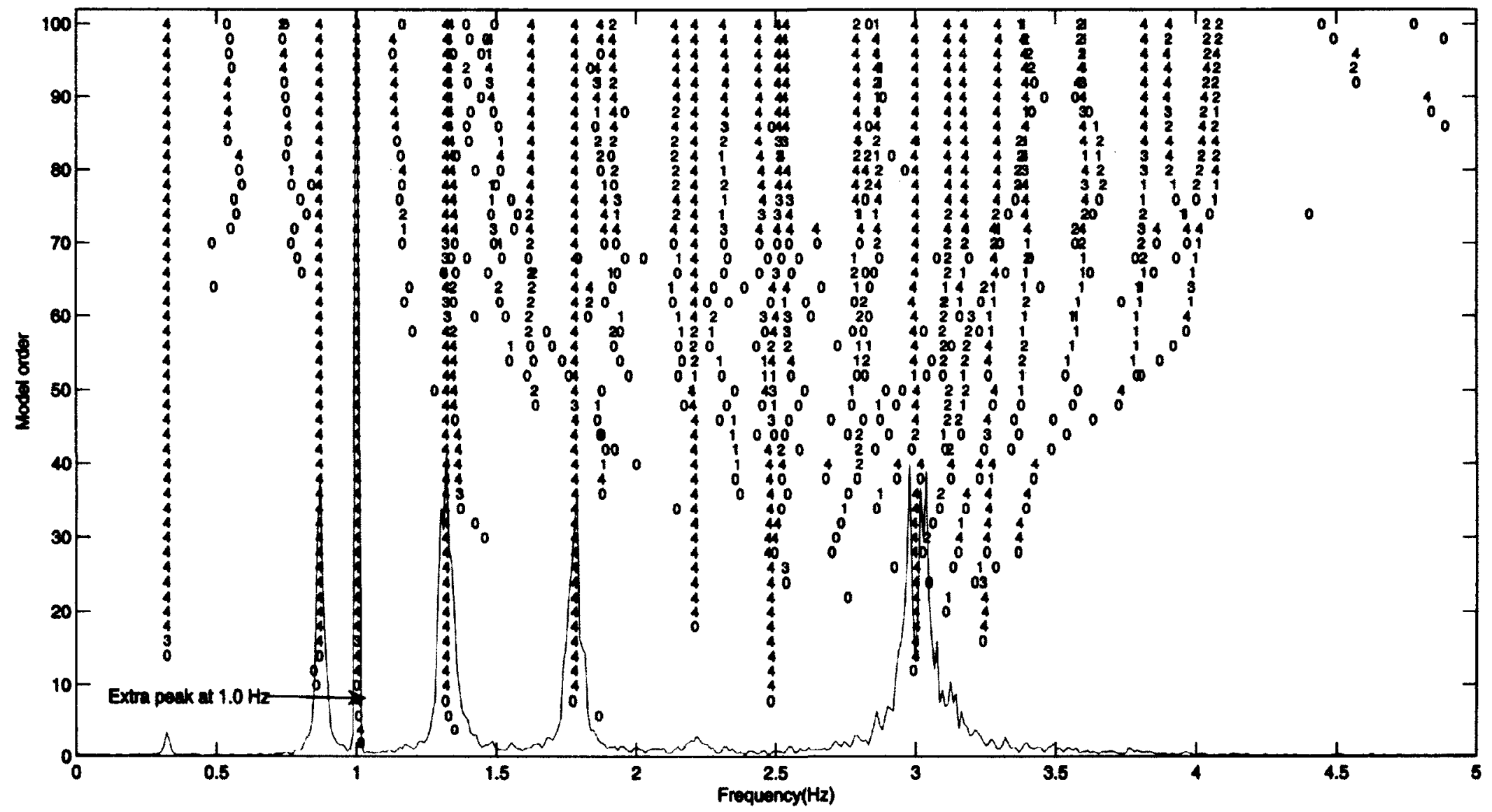

Figure 4-25: SSI stabilization diagram in the presence of harmonics. Extra peak at $1.0 \mathrm{~Hz}$ gives an indication of stable pole even though it is not a physical pole of the system. 


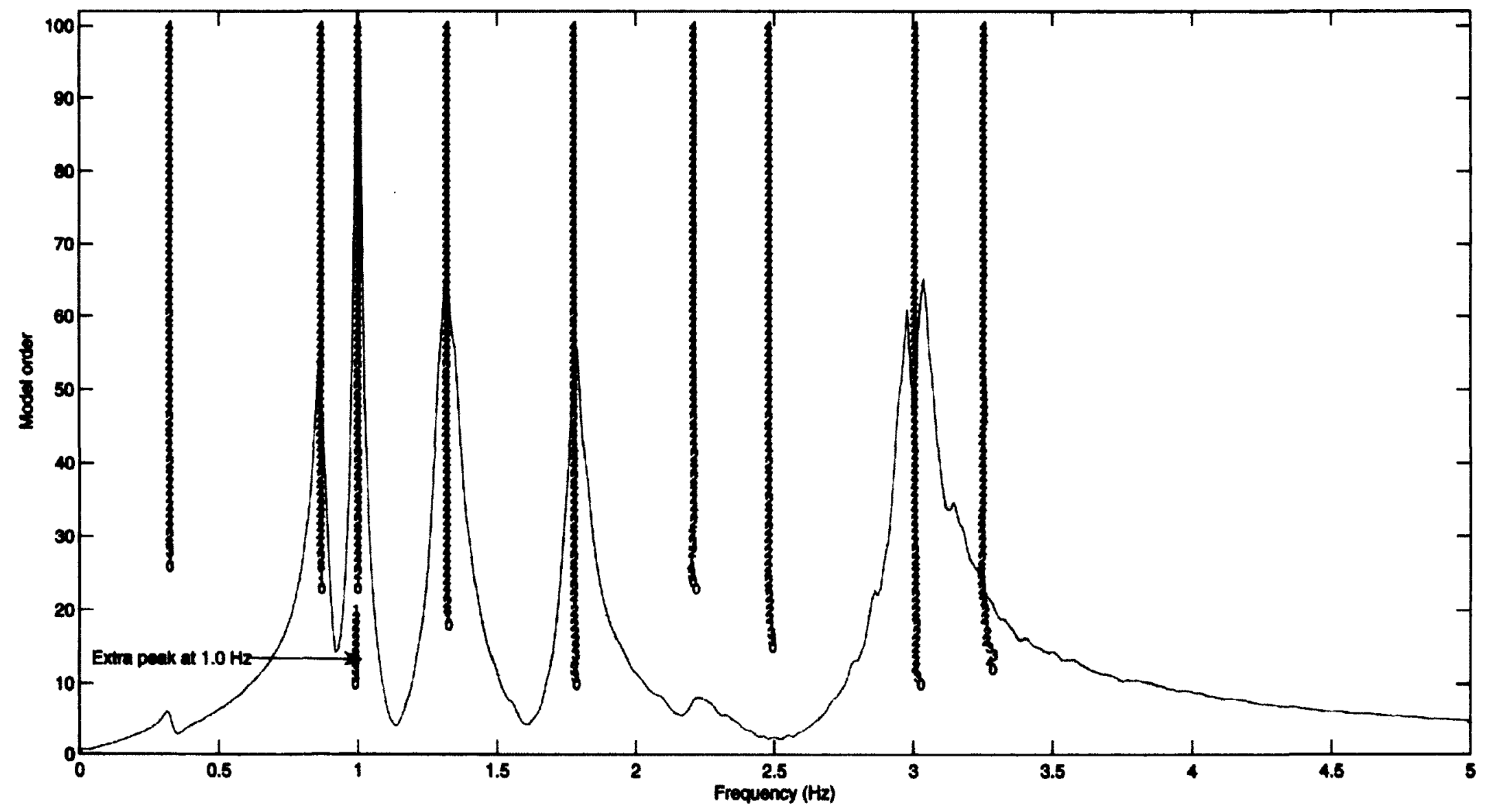

Figure 4-26: PolyMAX stabilization diagram in the presence of harmonics. Extra peak at $1.0 \mathrm{~Hz}$ gives an indication of stable pole even though it is a spurious pole. 


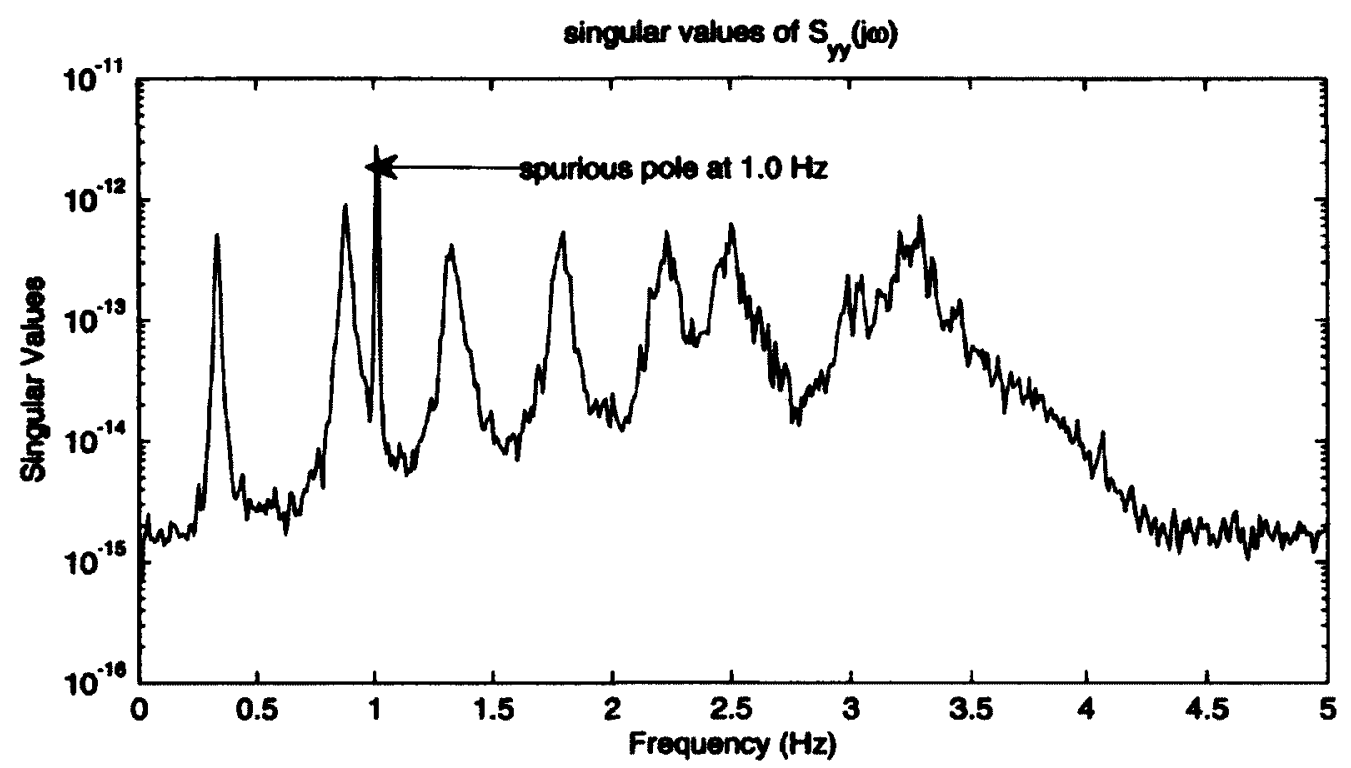

Figure 4-27: Singular value plot of FDD method. As it depends on the FFT of output signal, a peak will show up at $1.0 \mathrm{~Hz}$. And it is very hard to discriminate between physical pole and spurious pole boking at a peak of SVD plot.

\section{Case 2: Correlated and spatially incomplete excitation}

To investigate the effect of correlated and spatially incomplete excitation, the system is excited by omitting the input excitation at the third, fifth and seventh DOFs. Furthermore, the inputs at the remaining DOFs are constructed as follows to make them partially correlated:

$$
\begin{aligned}
& F_{1}=u_{1}+.1 u_{2}+.1 u_{4}+.1 u_{6}+.1 u_{8} \\
& F_{2}=.1 u_{1}+u_{2}+.1 u_{4}+.1 u_{6}+.1 u_{8} \\
& F_{4}=.1 u_{1}+.1 u_{2}+u_{4}+.1 u_{6}+.1 u_{8} \\
& F_{6}=.1 u_{1}+.1 u_{2}+.1 u_{4}+u_{6}+.1 u_{8} \\
& F_{8}=.1 u_{1}+.1 u_{2}+.1 u_{4}+.1 u_{6}+u_{8}
\end{aligned}
$$

The cross spectrum between first and second inputs are compared with the auto 
spectrum of first input (Figure 4-28). Unlike the previous case shown in Figure 4-5, due to the correlated nature of the input force the two spectra are approximately in the same order.

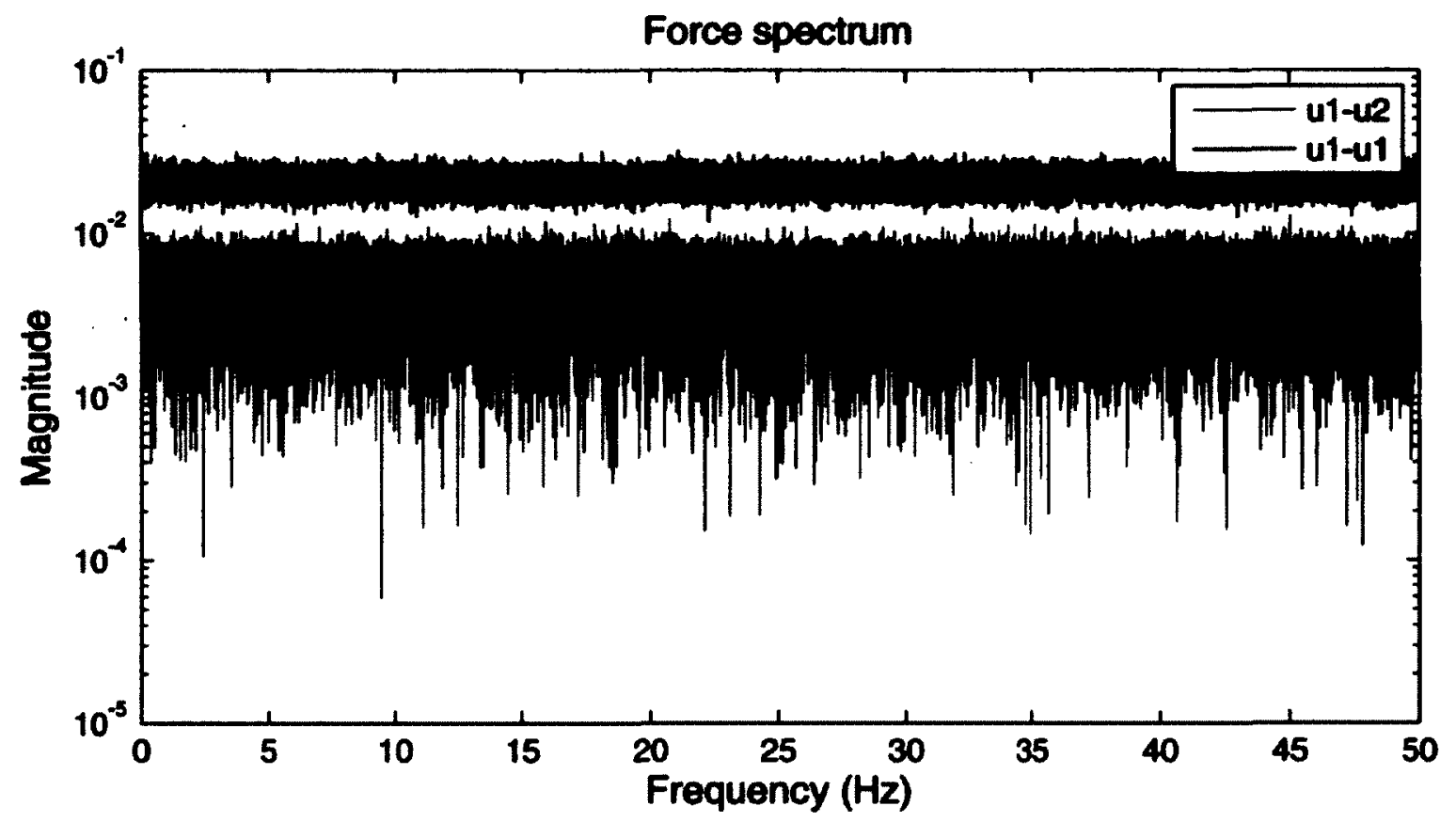

Figure 4-28: Comparison of force spectrum in case of partially correlated forces

The system identification results show that all the methods identify 8 modes with a high MAC and the error in frequency estimate is also negligible. But the damping estimate is affected more by the correlated input force. The result by the PolyMAX method is summarized in Table 4-4. It is observed that to better identify the low energy content mode; spectra computed using high correlation lag time is desirable. In this case, positive power spectra are computed with 1200 lag time and a $1 \%$ exponential window is employed to reduce leakage. The result from an uncorrelated 
and spatially complete excitation is also presented in Table 4-4 for comparison. Similar results are obtained by using other methods

Table 4-4: Effect of correlated force on damping estimate

\begin{tabular}{|c|c|c|c|c|c|c|c|c|}
\hline \multirow[t]{2}{*}{ Mode } & \multicolumn{4}{|c|}{ Uncorrelated and spatially complete excitation } & \multicolumn{4}{|c|}{ Correlated and spatially incomplete excitation } \\
\hline & Freqeuncy & Error(\%) & Damping(\%) & Error (\%) & Freqeuncy & Error(\%) & Damping(\%) & Error (\%) \\
\hline$\# 1$ & 0.3239 & 0.0035 & 1.29 & -0.7692 & 0.3236 & -0.0265 & 1.53 & 17.692 \\
\hline$\# 2$ & 0.8658 & -0.3643 & 1.31 & 26.053 & 0.8658 & -0.3643 & 1.38 & 32.789 \\
\hline$\# 3$ & 1.3171 & -0.3141 & 1.62 & 30.798 & 1.3188 & -0.1441 & 1.84 & 48.561 \\
\hline$\# 4$ & 1.778 & -0.1406 & 1.25 & -17.316 & 1.7768 & -0.2606 & 1.41 & -6.733 \\
\hline$\# 5$ & 2.1981 & -2.1821 & 1.35 & -25.000 & 2.2069 & -1.3021 & 1.08 & -40.000 \\
\hline$\# 6$ & 2.4816 & -0.3081 & 1.85 & -6.554 & 2.4802 & -0.4481 & 1.94 & -2.008 \\
\hline \#7 & 3.0105 & 1.2207 & 1.55 & -33.669 & 3.0073 & 0.9007 & 1.78 & -23.826 \\
\hline \#8 & 3.2562 & 0.8014 & 2.47 & -1.718 & 3.2525 & 0.4314 & 2.67 & 6.240 \\
\hline
\end{tabular}

\subsection{Damping estimate in OMA application}

It has been observed that even though frequency and mode shape estimate from system identification results are reasonably accurate, it is not the case for damping estimate. High bias and variance in damping estimate is quite evident. This situation is even worse if the basic OMA assumption of uncorrelated input is violated. The rather unrealistic damping estimation is primarily due to the random and bias errors associated with correlation and spectra approximation. Unlike frequency response function (FRF) in EMA, spectra in OMA are not unique unless the input is stationary and a sufficiently large number of averages are taken. Thus in general, for better power spectra estimation longer time history is required to average out the random errors. However, the bias errors, such as leakage cannot be reduced by averaging. In such cases, the popular Welch algorithm which utilizes window 
function along with averaging can be employed to reduce leakage error.

The use of cyclic averaging, along with asynchronous or synchronous averaging [64] is a powerful technique for reducing leakage error, as well as, random errors. Cyclic averaging reduces the leakage bias error by digitally filtering the data to eliminate the frequency information that cannot be described by the FFT completely prior to the application of FFT [65]. Practical implementation of cyclic averaging in EMA is presented in [66]. In this section, the effect of cyclic averaging is studied in the context of OMA application for better power spectra approximation which in turn, hopefully can lead to improvement on damping estimate.

\subsubsection{Cyclic averaging}

The cyclic averaging [4] is a special case of linear averaging in time domain where the digitization is coherent between cyclic averages. In the frequency domain, this is equivalent to a digital comb filter with passbands at frequency increments that are integer multiples of $\Delta f=1 / T$; thus resulting in attenuation between the passbands. The Fourier transform of an output time history is given by:

$$
X(\omega)=\int_{-\infty}^{\infty} x(t) e^{-j \omega t} d t
$$

The Fourier transform of the same time history shifted by $t_{0}$ :

$$
X(\omega) e^{-j \omega t_{0}}=\int_{-\infty}^{\infty} x\left(t+t_{0}\right) e^{-j \omega t} d t
$$




$$
(\omega) e^{\frac{j 2 \pi n}{T} t_{0}}=\int_{-\infty}^{\infty} x\left(t+t_{0}\right) e^{\frac{j 2 \pi n}{T} t} d t
$$

Note that in, Equation (4-3) the correction for the cases $t_{0}=N T$ where $N$ is an integer will be a unit magnitude with zero phase. Therefore, if each shifted history that is cyclic averaged occurs at an integer multiple of the observation period $T$, then the correction due to the time shift does not affect the frequency domain characteristics of the averaged result. The averaged time history is given by:

$$
\bar{x}(t)=1 / N_{c} \sum_{i=0}^{N_{c}-1} x_{i}(t)
$$

where $N_{c}$ is the number of cyclic averages. The Fourier coefficients $C_{k}$ of the averaged signal are:

$$
\begin{aligned}
& C_{k}=\frac{1}{T} \int_{0}^{T} \bar{x}(t) e^{-j \omega_{k} t} d t \\
& C_{k}=\frac{1}{N_{c} T} \int_{0}^{T} \sum_{i=0}^{N_{c}-1} x_{i}(t) e^{-j \omega_{k} t} d t \\
& C_{k}=\frac{1}{N_{c} T} \int_{0}^{N_{c} T} x(t) e^{-j \omega_{k} t} d t
\end{aligned}
$$

The above equations indicate that the Fourier coefficients of the cyclic averaged history spaced at $\Delta f=1 / T$ are the same Fourier coefficients from the original history spaced at $\Delta f=1 / N_{c} T$ which results in a higher frequency resolution. Note 
that Parseval's energy preservation theorem is not preserved by the cyclic averaging process since the frequency information not related to the harmonics of $\Delta f=1 / T$ is removed [67].

\section{Effect of cyclic averaging}

In order to study the effect of cyclic averaging, three cases of different signal processing of no cyclic averages, 2 cyclic averages and 4 cyclic averages are considered here. In all of these cases, the total measured time is constant. The spectra are computed with 2048 points of FFT and Hamming window with $50 \%$ overlap is employed in all the cases. Typical auto spectra obtained using these three signal processing are shown in Figure 4-29.

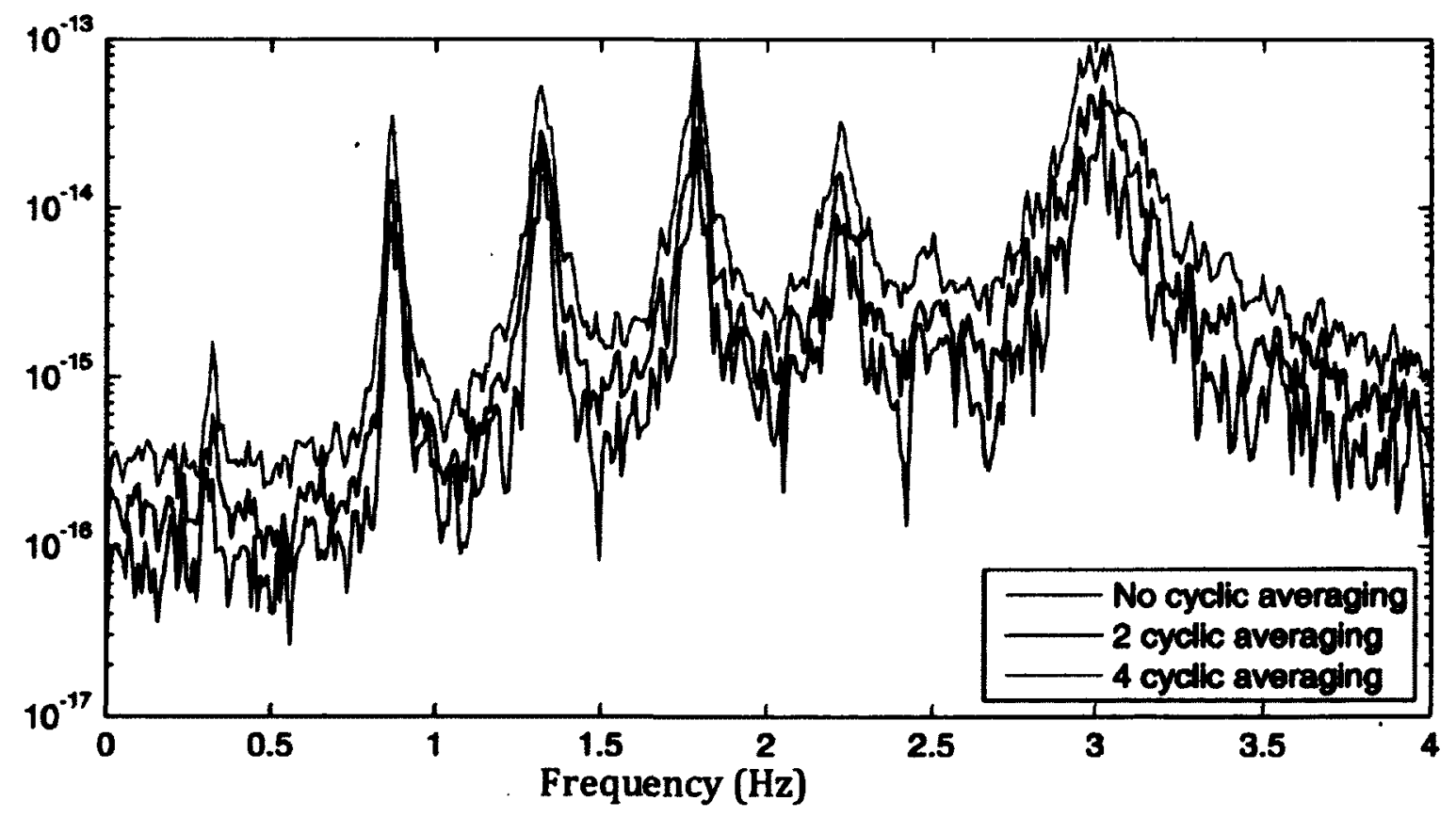

Figure 4-29: Effect of cyclic averaging for $1^{\text {st }}$ output location.

As indicated in Table 4-5, with no cyclic averaging, the error in damping estimates is significantly higher and in general, the damping is over-estimated in most cases. 
With 2 cyclic averages, the error is reduced for all the modes. Increasing the number of cyclic averages to 4 doesn't improve the overall results at all. This can be attributed to the considerably less number of spectral averages in case of 4 cyclic averaging compared to 2 cyclic averaging.

Table 4-5: Effect of cyclic averaging

\begin{tabular}{|c|cc|cc|cc|}
\hline & \multicolumn{2}{|c|}{ No cyclic averaging } & \multicolumn{2}{c|}{ 2 cyclic averaging } & \multicolumn{2}{c|}{ 4 cyclic averaging } \\
\hline Mode & $\begin{array}{c}\text { Damping } \\
(\%)\end{array}$ & $\begin{array}{c}\text { Error } \\
(\%)\end{array}$ & $\begin{array}{c}\text { Damping } \\
(\%)\end{array}$ & $\begin{array}{c}\text { Error } \\
(\%)\end{array}$ & $\begin{array}{c}\text { Damping } \\
(\%)\end{array}$ & $\begin{array}{c}\text { Error } \\
(\%)\end{array}$ \\
\hline$\# 1$ & 2.28 & 75.63 & 1.71 & 31.67 & 1.75 & 34.40 \\
$\# 2$ & 1.26 & 20.94 & 1.09 & 4.91 & 0.82 & -20.72 \\
$\# 3$ & 1.98 & 59.90 & 1.67 & 35.15 & 0.58 & -53.48 \\
$\# 4$ & 1.56 & 3.37 & 1.54 & 2.12 & 0.75 & -50.46 \\
$\# 5$ & 1.84 & 2.14 & 1.84 & 2.25 & 0.55 & -69.19 \\
$\# 6$ & 2.08 & 5.26 & 2.06 & 4.08 & 0.95 & -51.90 \\
$\# 7$ & 0.52 & -77.86 & 3.65 & 56.38 & 2.45 & 4.97 \\
$\# 8$ & 1.37 & -45.52 & 2.14 & -15.01 & 0.82 & -67.18 \\
\hline
\end{tabular}

In this study, a total 700 s time history is used. By increasing the time duration to 1400 s, a similar result is obtained i.e. higher number of cyclic averaging does not guarantee better damping estimation. Even though cyclic averaging is mentioned [65] as an approach for reduction of leakage, it follows from the results that proper precautions should be taken to select the optimum number of cyclic averaging. It should be noted that FDD is used in parameter identification process.

\subsubsection{Use of an exponential window}

Exponential window is often used as a signal processing technique for reducing leakage in spectra computation. Moreover, the introduced bias by using exponential 
window is predictable and can be corrected unlike the use of other windows. At the same time, in order to make sure that enough information of the lower frequency mode is present in the analysis; longer correlation lag time should be used. The effect of longer correlation lag time on damping estimate is obvious from the results presented in Table 4-6. The analysis is carried out by PolyMAX method with $1 \%$ exponential window.

Table 4-6: Effect of correlation lag time on damping estimate

\begin{tabular}{c|cc|cc|cc|}
\hline Mode & \multicolumn{2}{|c|}{ 256 lag time } & \multicolumn{2}{c|}{ 1024 lag time } & \multicolumn{2}{c|}{ 2048 lag time } \\
\hline & Damping (\%) & Error (\%) & Damping (\%) & Error (\%) & \multicolumn{2}{|c|}{ Damping (\%) } \\
\hline$\# 1$ & 1.75 & 34.82 & 1.29 & -0.77 & 1.29 & -0.50 \\
$\# 2$ & 1.41 & 35.89 & 1.31 & 26.05 & 1.31 & 25.84 \\
$\# 3$ & 1.79 & 44.20 & 1.62 & 30.80 & 1.62 & 31.11 \\
$\# 4$ & 1.59 & 5.27 & 1.25 & -17.32 & 1.60 & 5.97 \\
$\# 5$ & 1.62 & -9.95 & 1.35 & -25.00 & 1.70 & -5.56 \\
$\# 6$ & 1.98 & 0.08 & 1.85 & -6.55 & 2.08 & 4.88 \\
$\# 7$ & 1.81 & -22.68 & 1.55 & -33.67 & 2.07 & -11.42 \\
$\# 8$ & 2.31 & -8.22 & 2.47 & -1.72 & 2.47 & -1.92 \\
\hline
\end{tabular}

\subsection{Conclusion}

This chapter presented the results of a comparison study of the performance of four stochastic system identification methods by Monte-Carlo simulation. In terms of frequency and mode shape estimation, all the methods provide accurate results. The comparatively high bias in the mode shape estimation by PolyMAX method can be 
attributed to the biased spectra estimation by the correlogram approach. In case of damping estimate, the parametric identification methods of SSI and PolyMAX outperform FDD and ERA methods although in all cases the effect of high bias and variance is obvious. Moreover, both the SSI and PolyMAX methods are not robust for identifying less excited modes.

The effects of violating OMA assumptions are also studied. It is found that if uncorrelated white noise assumption is not met, the errors in damping estimate can significantly increase. Furthermore, none of the methods are capable of discriminating between harmonic frequency and eigenfrequency.

To estimate leakage free spectra, currently available signal processing techniques are explored. Apparently, use of cyclic averaging with windowing and overlapping lead to a better estimation of the spectra but the leakage effect is not entirely eliminated. Furthermore, one should exercise judgment in selecting the number of cyclic averaging as high cyclic averaging gives as good as or comparatively inferior results to the case where no cyclic averages are used. Meanwhile, exponential window with a considerably longer correlation lag time results in a comparatively better estimation of damping. It will be a fair conclusion to say that more research effort is necessary for leakage free spectra estimation in case of OMA application. This is essential to achieve a better understanding of the OMA procedure which in turn helps in successful real life implementation of OMA techniques. 


\section{Chapter 5 - Application to Civil}

\section{Structures}

\subsection{Introduction}

Civil Structures, especially bridges, are one of the major OMA application areas for dynamic characterization, damage detection as well as condition assessment. This Chapter presents the OMA related studies carried out on the Confederation Bridge, in Canada. The Confederation Bridge at 12.9 kilometers is the world's longest bridge over ice covered sea-water. A permanent monitoring network installed on the bridge captures the vibration responses of the structure resulting from ambient excitations such as wind, traffic, ice and earthquake loads. This chapter focuses on various aspects of operational modal analysis; collection of ambient vibration data, building a software platform for rapid and efficient handling of the data, parameter estimation techniques used and validation of results and comparison with FE model. 


\subsection{Confederation Bridge}

The Confederation Bridge is a prestressed concrete box girder structure, spanning the Northumberland Strait, linking Borden, Prince Edward Island, and Cape Tormentine, New Brunswick in Eastern Canada. To cover the $12.9 \mathrm{~km}$ long shore-toshore distance the bridge is divided into 21 approach spans, two transition spans of $165 \mathrm{~m}$ each and 43 main spans of $250 \mathrm{~m}$ each at a typical height of $40 \mathrm{~m}$ above the mean sea level. The main-span portion of the bridge is comprised of 22 repetitive structural frame modules of $500 \mathrm{~m}$ length each. Each module is composed of a $\mathbf{4 4 0}$ m portal frame, made up of a $250 \mathrm{~m}$ centre span and two $95 \mathrm{~m}$ overhangs, one on each side of the centre span, plus a $60 \mathrm{~m}$ simply supported drop-in expansion span (Figure 5-1). The portal frame is constructed of four types of prefabricated component units, pier base, pier shaft, main girder and fixed drop-in span girder. A continuous frame structure is constructed by post-tensioning of the individual structural pieces together. The bridge girders are single cell trapezoidal box girders with section depth varying from $14 \mathrm{~m}$ above the piers to $4.5 \mathrm{~m}$ at mid-span of the drop-in girder, and a cross-section width varying from $5.0 \mathrm{~m}$ at the bottom to $7.0 \mathrm{~m}$ at the top, with $2.5 \mathrm{~m}$ deck overhangs. 


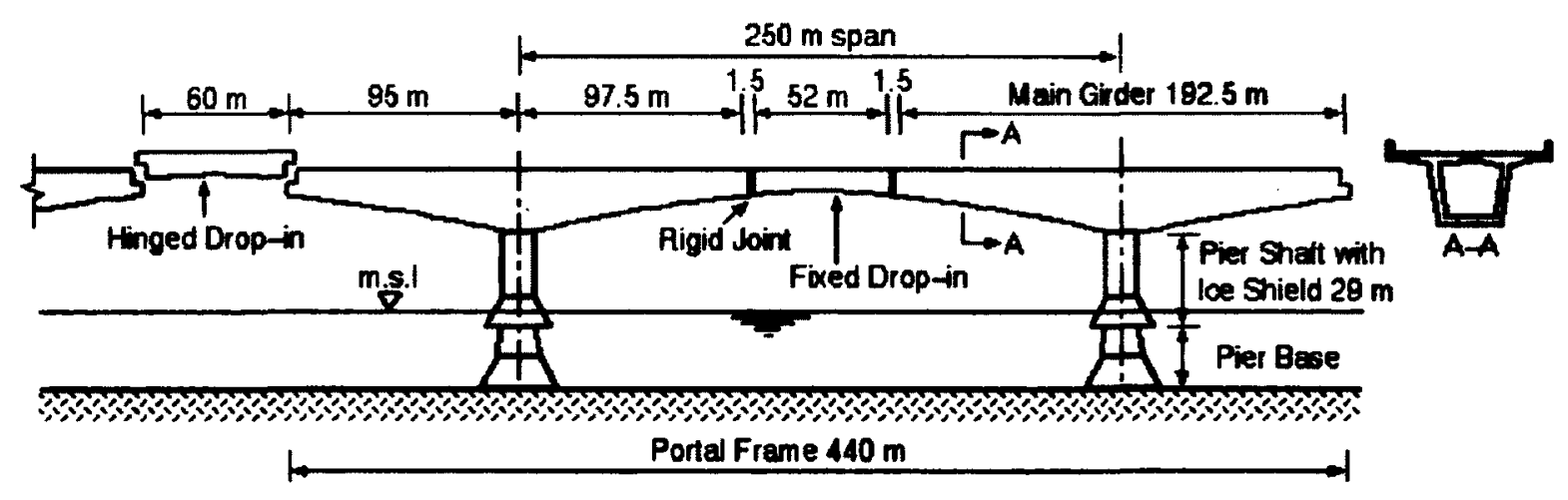

Figure 5-1: Typical frame layout of Confederation Bridge

\subsection{Continuous monitoring system}

A comprehensive long-term monitoring system on the Confederation Bridge has been in operation since the bridge opening in 1997 in order to collect data and information about its behaviour and performance. The monitoring system measures and records both environmental and bridge response data related to ice forces, short and long-term deflections, thermal effects, corrosion and dynamic responses. The sensors of the monitoring system are installed on two adjacent spans of the bridge, a drop-in span and a rigid frame. The dynamic responses of the structure, due to the effects of wind, traffic, ice floe, and earthquake loadings are captured by 62 accelerometers distributed over the instrumented section of the bridge. In this study, the recorded signals from $\mathbf{5 0}$ of these accelerometers are used, which are shown in Figure 5-2. Vibration responses of the bridge girders are measured in the 
vertical and lateral horizontal (herein forth referred simply as "lateral") directions, as shown in Figure 5-2. This setup facilitates the recovery of vertical bending, lateral bending and torsional vibration modes of the bridge superstructure. The vibration sensors used in the monitoring system include both piezo-electric accelerometers and servo accelerometers. The measured analog signals are conditioned and filtered for anti-aliasing by a Frequency Devices 8-pole $50 \mathrm{~Hz}$ low-pass Bessel filter. Signals are then digitized at sampling frequency of $125 \mathrm{~Hz}$ by a network of high-speed Campbell Scientific data loggers before being sent to on-site computers and transmitted back to Carleton University in Ottawa for data archival and research. Dedicated communication lines between the different data loggers ensure simultaneous triggering and recording. The data loggers operate in continuous buffered data collection mode, which upon triggering by detection of specific dynamic events, such as heavy traffic or high winds, or simply upon user request, store a ten minute data set including a thirty second pre-triggering buffer. Otherwise, only statistic information determined from the time histo ry data, such as mean, maximum, minimum and standard deviation, are stored. Additional details of the monitoring system setup have been described in [68] [69]. 


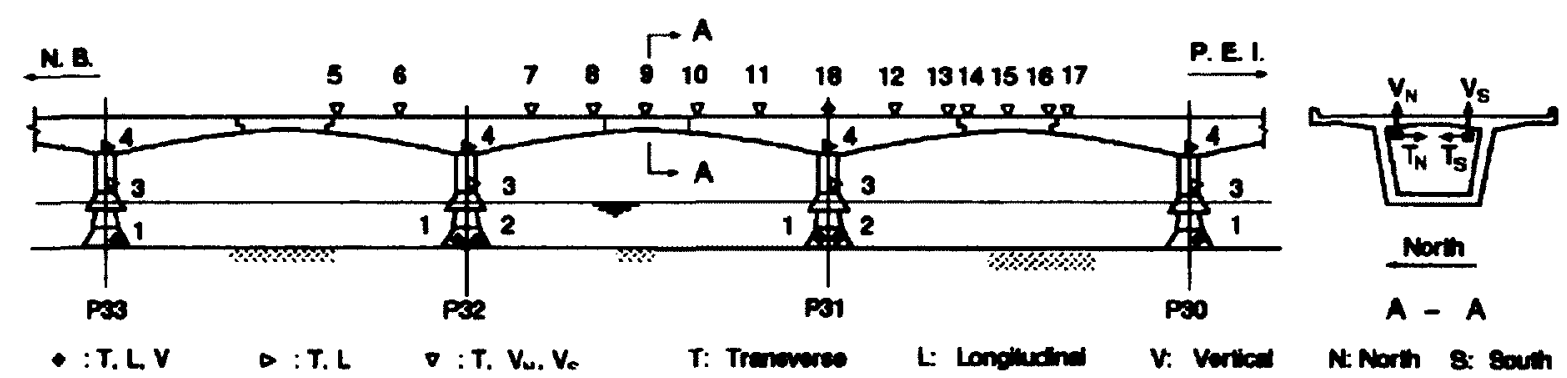

Figure 5-2: Locations of accelerometers in Confederation Bridge monitoring system

\subsection{Software platform}

In order to minimize the effort required for processing of large volume of data collected by the Confederation Bridge monitoring project, a real-time data processing and analysis application platform has been developed which encompasses all aspects of data manipulation [70]. This robust modular software, Signal Processing Platform for Analysis of Structural Health (SPPLASH), has a modular design in which each module has its own GUI for configuration of the processing of data and is designed to interface seamlessly with other appropriate modules in the application platform. The software platform consists of modules for data processing, data display and plotting, system identification and visualization of bridge response, all integrated through graphical user interfaces (GUIs). The applications are designed and adapted to run in a real-time mode by automatically sorting incoming data and re-directing it to the processing and animation modules 
for graphic display of bridge displacements and motion in near real-time, as limited by the network speed.

In a vibration monitoring system, acceleration data after analog-to-digital (A/D) conversion by the data loggers, often require some processing to prepare the data for analysis and interpretation. Typical issues which must be resolved for data collected from an instrumented structure are:

- A lack of synchronization of data time-stamps is possible as a result of the data being recorded by multiple data loggers.

- Due to the high sampling rate necessary to capture all information, small gaps may be present in the data. Gaps may occur if the CPU of the data logger is overworked during the recording of an event.

- Duplication of data samples is possible due to an error in the acquisition software.

- An offset as well as high frequency noise may be present in the data.

- Data from a particular event may be separated into different files if an event occurs during a prescheduled automatic collection of the data from a logger.

- Multiple events can be stored in a single file.

SPPLASH has been developed with the necessary algorithms to correct the aforementioned errors. The correction of these errors has been subdivided into two categories, pre-processing and processing. The pre-processing algorithms are used solely for the organization of the data, i.e. no changes are made to the collected data 
samples and the processing algorithm performs necessary corrections where applicable. The pre-processing of data is achieved through the following steps:

- Complete datasets are extracted from the collected data files to form a single dataset.

- Data files are converted to the necessary format for further processing and analysis.

- Complete data events are formed by assembling matching data sets of proper duration.

Processing of data comprises of the following steps:

- A Piecewise Cubic Hermit Interpolating Polynomial is employed to patch small gaps that may be present in the preprocessed data, using four samples before and after the gap.

- Searching for duplicate data point is carried out by comparing less than zero sampling intervals within the data file. If duplication is present, the record is removed from the data file.

- The baseline offset of a sensor is removed by determining the mean of the signal and purging it from the record.

- Data samples are converted from measured voltage readings to engineering unit of $\mathrm{m} / \mathrm{s}^{2}$ by proper conversion factor related to each sensor.

- As the data event consists of datasets from multiple data loggers, a consistent sampling rate is employed. The minimum sampling rate from the data loggers under consideration is determined, and all loggers with a 
higher rate are down-sampled. Prior to downsampling, data is filtered by a $12^{\text {th }}$ order Chebyshev type I low-pass filter to prevent aliasing.

- A typical sampling rate for dynamic data is $125 \mathrm{~Hz}$, whereas the frequency range of interest is commonly below $15 \mathrm{~Hz}$, computational loads can be reduced through decimation of the data. In order to prevent aliasing during this process, the same low-pass Chebyshev type I filter is employed.

- The acceleration data is doubly integrated, through cumulative trapezoidal numerical integration, to obtain displacement data of the structure, which are utilized in the visualization module.

Following the processing of monitoring data, analysis tools are utilized to extract relevant engineering information of the monitored structure. As a preliminary analysis of the bridge monitoring response signals, power spectral density (PSD) analysis of the monitoring data can be conducted to identify the dominant structural vibration frequencies and distribution of the energy of the signals in the frequency domain. However for more accurate and thorough analysis more sophisticated system identification techniques, SSI and p-LSCF have been implemented in SPPLASH.

To facilitate the interpretation of the extracted information from the monitoring data, SPPLASH has incorporated a visualization module with 3D model representation of the structure that displays the response of the structure at the model nodes through interpolation of the response at sensor locations. Along with displacements of the structure, experimental and analytical mode-shapes can be 
visualized through use of the GUI. This ability can provide significant clarity when attempting to distinguish between vibration modes of similar frequencies. A more in-depth description can be found in [71].

\subsection{Typical vibration behavior}

Twenty-one datasets of vibration responses of the Confederation Bridge under different dynamic loading scenarios collected by the continuous monitoring system have been analyzed and the results are presented in [35] [72]. Selection of these data sets are according to strict criteria to ensure that they are representative of each of the loading scenario considered, i.e. "ambient", "high wind", "traffic", and "ambient with ice". Each dataset comprises the simultaneous acceleration timehistory records from a total of $\mathbf{5 0}$ accelerometers, which are permanently installed at monitoring locations 1 through 18 between Piers 31 and 32. It has been reported that the frequency content of responses collected under high wind scenarios are characterized by having most of the response energy in the low frequency range, mostly below $1.0 \mathrm{~Hz}$. For the case of traffic loading, the vibration response is dominated by higher frequency modes, particularly those in the 2.5 to $3.5 \mathrm{~Hz}$ range in the vertical direction, and in the 12 to $14 \mathrm{~Hz}$ in the transverse direction which are typical of bridge vibration modes excited by the interaction effects between heavy vehicle and the bridge structure. In the lateral direction, $3.3 \mathrm{~Hz}$ corresponds to the first torsional mode of the bridge; however it involves significant associated lateral 
deflections. Under the more commonly encountered loading scenario of combined typical ambient wind and traffic excitations, the vibration energy in the vertical direction is now distributed between the low-frequency wind-excited modes and the mid frequency traffic excited modes up to around $5.2 \mathrm{~Hz}$. The frequency content of the ambient response in the presence of ice floes is not too dissimilar to the response under the ambient scenarios without ice. Some vibration modes in the vertical direction of the response, such as the one at $4.15 \mathrm{~Hz}$, are more clearly excited under the loading scenarios with ice floes than under the other loading scenarios.

\subsection{Monitoring datasets}

A total of 14 data sets, sampled at $125 \mathrm{~Hz}$, have been selected from the Confederation Bridge monitoring database for the present study. 12 of these datasets are recorded within a short period of time ( 2 days) during which the environmental conditions and loading scenarios can be considered constant. Figure (5-3) shows that the average temperature of the concrete at the instrumented section of the bridge and the wind speed at the bridge site are relatively consistent for the first 12 datasets. The range of average concrete temperature is $-2.7^{\circ} \mathrm{C}$ to $1.4^{\circ} \mathrm{C}$, while the average wind speed range is $7.2 \mathrm{~m} / \mathrm{s}$ to $14.8 \mathrm{~m} / \mathrm{s}$. The ranges of these values, $1.3^{\circ} \mathrm{C}$ and $7.6 \mathrm{~m} / \mathrm{s}$ are reasonable when compared to the yearly variations of $45^{\circ} \mathrm{C}$ and $30 \mathrm{~m} / \mathrm{s}$ for the conditions. The remaining 2 events are 
Chapter 5 Application to civil structures

characterized by high wind scenario. The average wind speed was $35 \mathrm{~m} / \mathrm{s}$ and the bridge was remained closed for traffic during these wind storms.

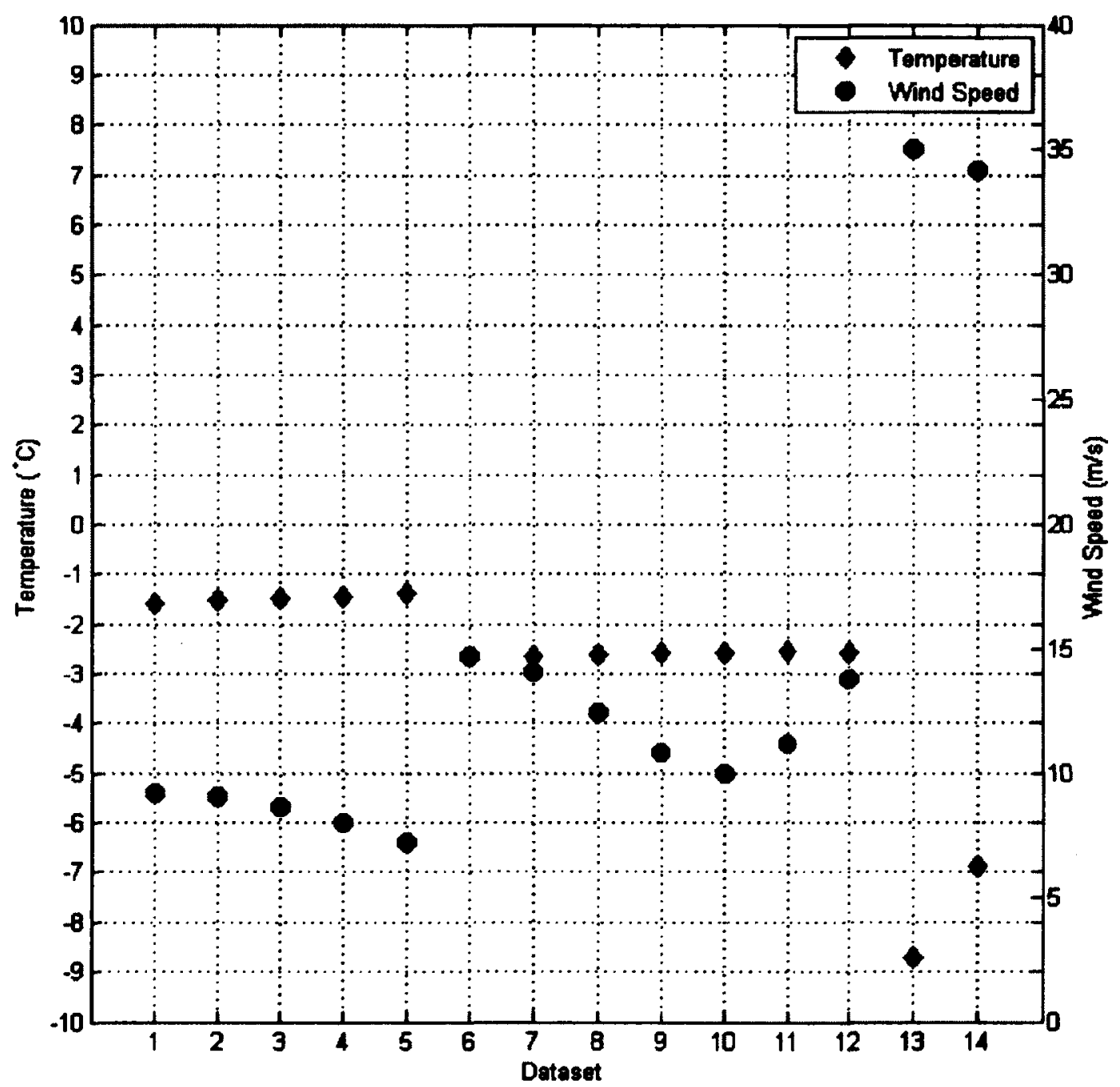

Figure 5-3: Average concrete temperature and average wind speed of 14 datasets 


\subsection{System identification}

The following processing tasks are carried out to prepare the data for system identification:

- Baseline adjustment of the acceleration time histories by removing any voltage drift from the accelerometer signals.

- Low pass filtering with a cut off frequency of $9 \mathrm{~Hz}$ using a Chebyshev type II filter of order 12. This eliminates the high frequency noise and signal components, enhancing response signal in the band of interest $(0-5.5 \mathrm{~Hz})$. The filtering is performed in the forward and reverse directions to eliminate nonlinear phase distortion. The low-pass filtering also serves as an antialiasing filter for the subsequent re-sampling of the data.

- Down-sampling of the data to one third of the original sampling rate of $125 \mathrm{~Hz}$ corresponding to a sampling time of $24 \mathrm{~ms}$. For Frequency Domain Decomposition (FDD); to get an enhanced frequency resolution, data is down sampled 5 times resulting in a sampling interval of $40 \mathrm{~ms}$.

The above processing tasks, as well as the system identification analysis of the data, are carried out using monitoring software developed for the Confederation Bridge project by SPPLASH.

\subsubsection{Stochastic subspace identification (SSI)}

4 vertical and 2 lateral sensors at monitoring locations 7 and 9 are used as reference sensors for cross-correlation computation. These sensors are suitable references 
because of their relatively high response amplitudes and because their locations do not simultaneously coincide with modal nodes of any of the important vibration modes of the bridge. High speed FFT based method is used to estimate correlations (Chapter 3). In the present study, proper models were identified by the SSI method with a model order of $n_{\max }=150$ i.e. models containing 75 modes. Even though the actual model order of the data analyzed here is typically around 40 , a relatively high maximum model is used in the construction of the stabilization diagrams to allow for a clear visualization of stabilized trends. The number of time lags of the data correlations used in the construction of the Toeplitz matrix (Equation 3-22), which forms the basis of the system identification, is taken as $i=\mathbf{2 5 0}$ for all datasets analyzed corresponding to $12 \mathrm{~s}$ of "free" vibration response time. This value of $i$ not only satisfies mathematical requirement of $r \times i>n_{\max }$ but also ensures that the data correlations of the Toeplitz matrix span more than a full vibration cycle of the lowest mode. To stabilize modal parameters, $0.5 \%$ is limit is chosen for frequency, whereas for mode shape and damping the limits are $1 \%$ and $15 \%$ respectively. Figure 5-4 shows the stabilization diagram for dataset 1. 


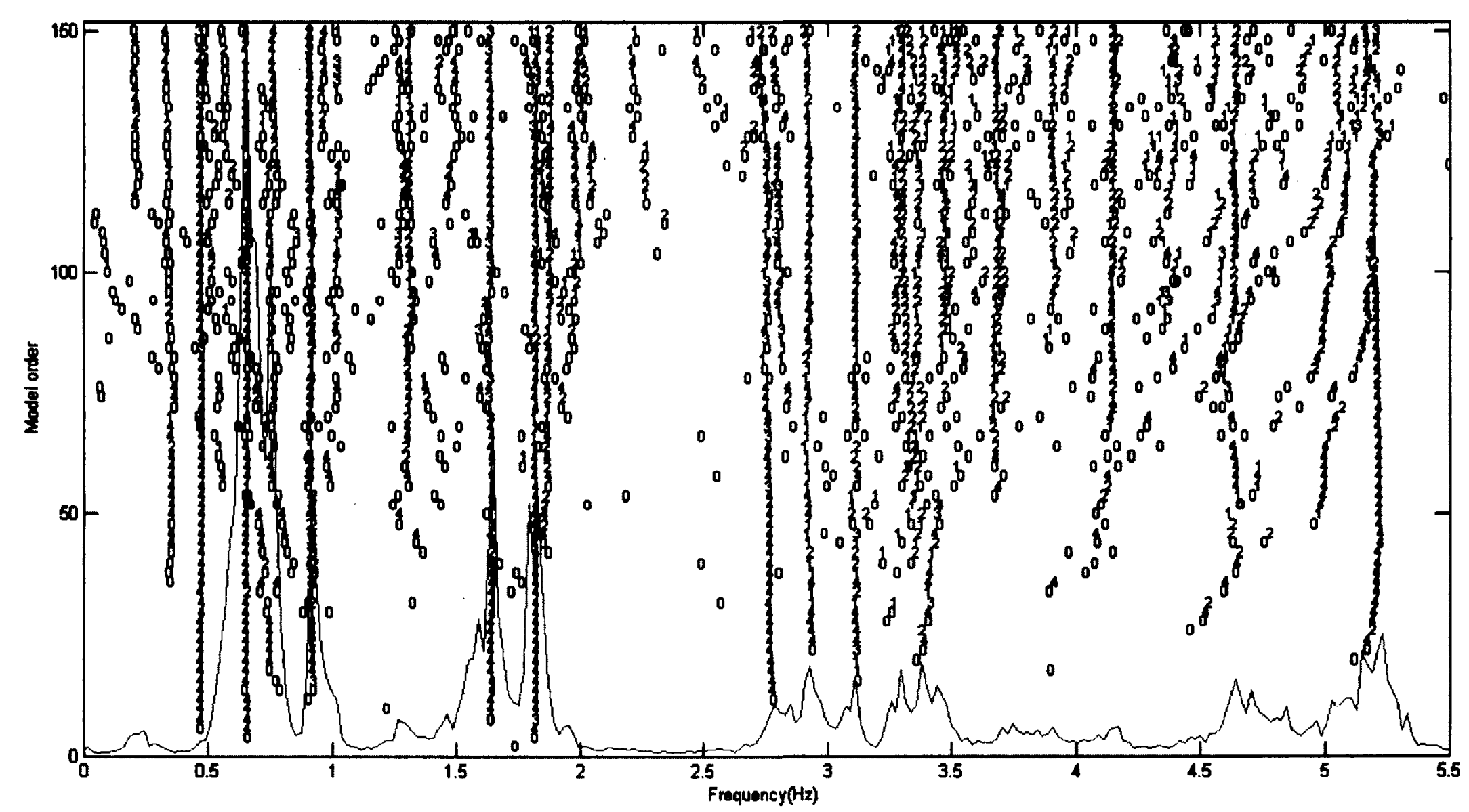

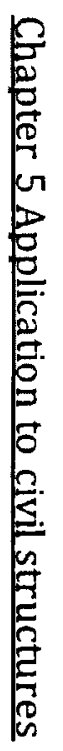

Figure 5-4: Stabilization diagram for SSI method. Here "4" stands for stable pole, " 3 "stands for frequency and mode stable, " 2 " stands for frequency and damping stable and " 0 " stands for new frequency 


\subsubsection{Polyreference Weighted Least-Squares Complex Frequency Domain Estimator (PolyMAX)}

Here, the primary identification data, positive power spectrum is estimated via correlogram approach with 2048 correlations lag time. The same 6 sensors (4 vertical and 2 lateral) at monitoring locations 7 and 9 are used as reference sensors. To reduce the leakage $1 \%$ exponential window is applied prior to correlogram spectrum estimation. For producing the stabilization diagram, the model order is selected in the range of $\mathbf{1 5 0 - 2 0 0}$ and the same criteria like SSI method is employed: $0.5 \%$ for frequency, $1 \%$ mode shape and $15 \%$ for damping. Figure $5-5$ shows the stabilization diagram for $1^{\text {st }}$ dataset. The stables poles can be easily picked from clear stabilization diagram. Interesting to note that there are gaps in some of the vertical line indicating these frequencies are not being identified within a specific band of model order. Furthermore, PolyMAX needs higher model order to stabilize poles. 


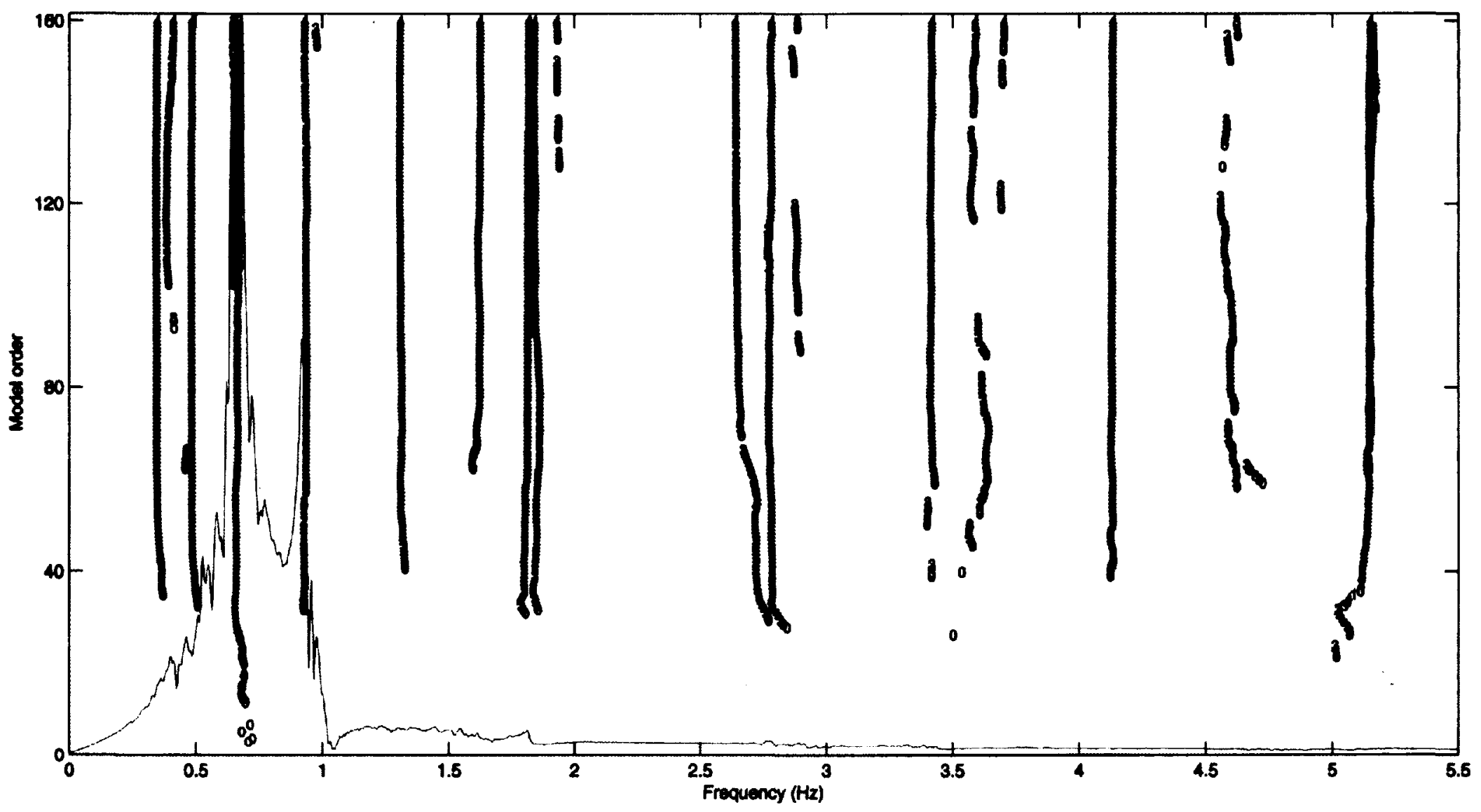

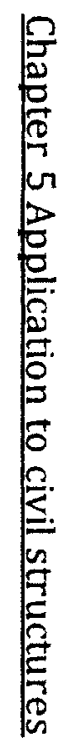

Figure 5-5: Clear stabilization diagram for PolyMAX method. Here " 4 " stands for stable pole, " 3 "stands for frequency and mode stable, " 2 " stands for frequency and damping stable and " 0 " stands for new frequency 


\subsubsection{Eigensystem Realization Algorithm (ERA)}

For ERA, the correlations calculated with respect to same 6 sensors at monitoring locations 7 and 9 (4 vertical and 2 lateral sensors) are used as identification data. Hankel matrix is formed with 600 columns and 8750 rows that is equivalent to $9.3 \mathrm{~s}$ of "free response" data. In most of the datasets, the truncation of block Hankel matrix is carried out with 50-60 model order so 25-30 modes are identified (Figure 5-6). The systematic elimination of doubtful modal properties is carried out as follows:

- As the frequency range of interest is 0-5.5 Hz, only frequencies below $5.5 \mathrm{~Hz}$ are taken into account.

- Modes with high damping ratio are discarded. A 5\% threshold is established for the present study.

- Repeated modes are eliminated based on the lowest energy content given by the singular values. Frequencies within $20 \%$ of each other and MAC value of 0.8 are used to identify such modes.

As shown in Figure 5-6, there is no clear "gap" in singular value i.e. it is very difficult to determine the number of modes being excited by looking at singular value plot. An initial guess of model order can be carried out by looking at singular value plot of the spectra matrix, just like Frequency Domain Decomposition (FDD) method. 


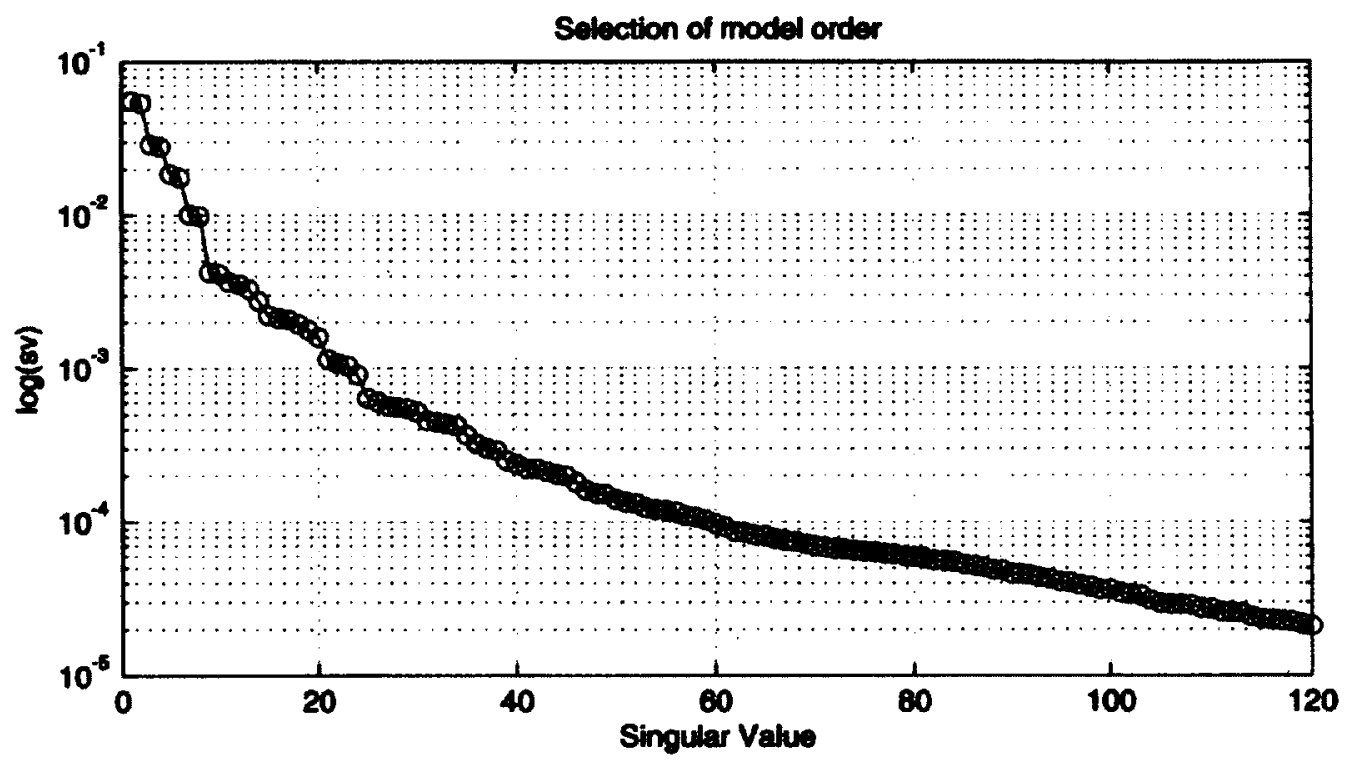

Figure 5-6: Singular vale of block Hankel matrix. As it can be seen, there is no clear "gap" or sudden drop in singular vale. Truncation is carried out with 50-60 model order to make sure all the modal information is present in the output

\subsubsection{Frequency Domain Decomposition (FDD)}

The spectra are estimated via Welch method [38] using 2048 points FFT with a Hamming window of $50 \%$ segment overlap. This results in a frequency resolution of $0.0122 \mathrm{~Hz}$ for singular value plot. For each dataset, two spectral matrices were calculated, one for vertical and another one for lateral accelerations. Then, a singular value decomposition of the spectral matrices was performed to evaluate the corresponding non-zero singular values. Figure 5-7 shows the average singular values of all datasets for both directions. Damping estimate is performed in time domain assuming a SDOF around a peak via enhanced FDD. 

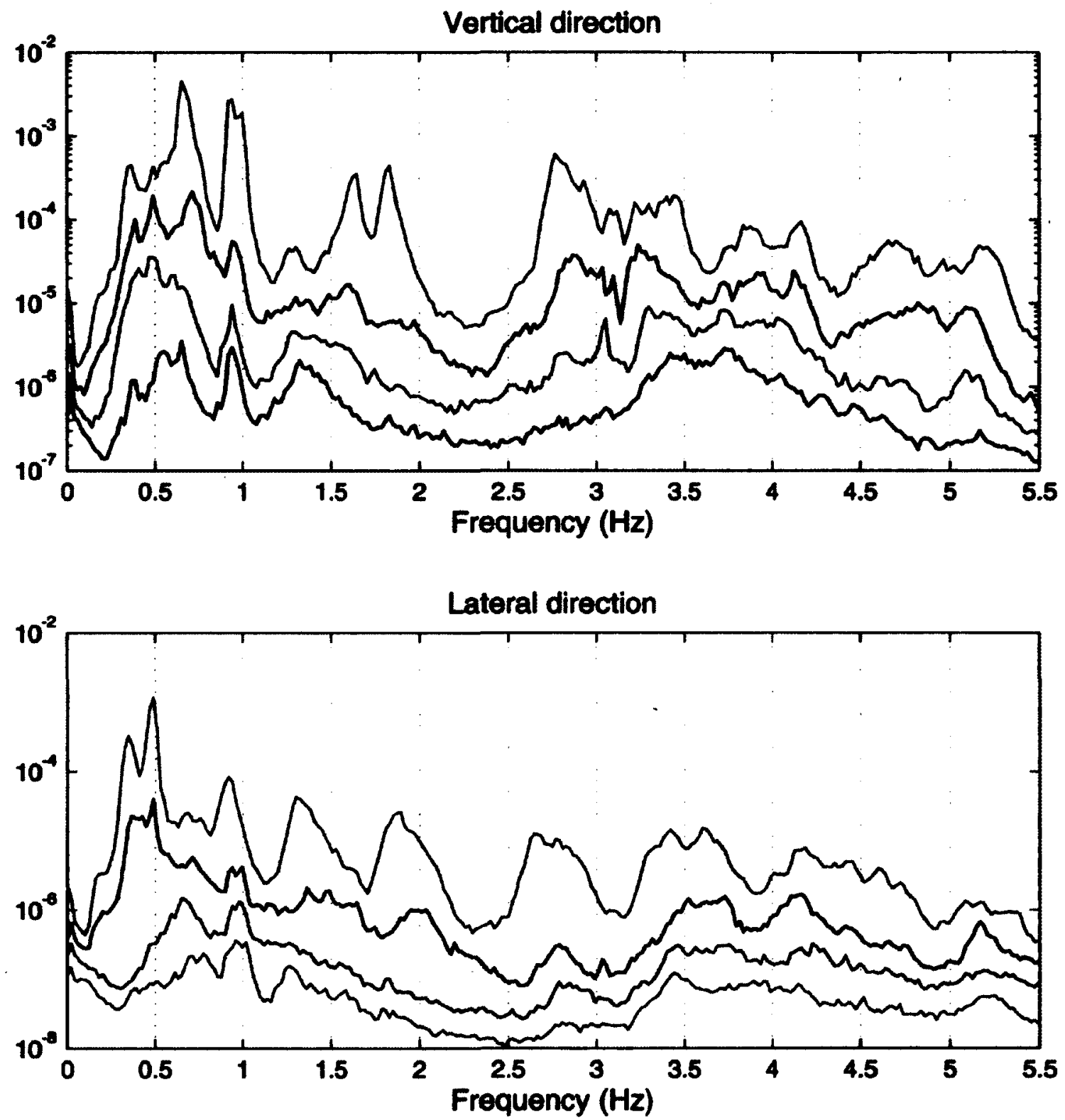

Figure 5-7: Average singular values of the spectra matrices for both vertical and lateral direction 


\subsection{Analysis results from monitoring data}

Modal vibration frequencies, mode shapes and damping ratios obtained by four system identification methods of the fourteen monitoring datasets described earlier are presented here. The results are also compared to the theoretical design values based on the finite element model to examine the correlation between extracted and analytical modal properties

\subsubsection{Modal frequencies}

Table 5-1 presents a summary of the modal frequencies extracted from the monitoring data and the corresponding values from the shell finite element models. Both SSI and PolyMAX algorithms are able to identify 21 modes below $5.5 \mathrm{~Hz}$. ERA method fails to detect a mode at $0.55 \mathrm{~Hz}$ whereas in case of FDD method; two modes namely at $0.55 \mathrm{~Hz}$ and $0.84 \mathrm{~Hz}$ are not estimated. The finite element modal frequencies based on the field measurement of concrete modulus $\left(E_{c}=43 \mathrm{GPa}\right)$ are in close agreement to the measured values with an overall observation of estimated frequencies being slightly lower than the theoretical frequencies. Furthermore, the good agreement between the measured modal frequencies and the updated finite element model values shows that the structure's dynamic properties of stiffness and mass are reasonably close to the design values.

In Table 5-1, the column labeled "number of identification" lists the number of monitoring datasets from which the corresponding eigenfrequencies and mode 
shapes have been identified, out of the total 14 datasets considered. The numbers give an indication of how frequently the vibration modes are being well excited under the typical loading scenarios and thus participate significantly in the measure structural responses. The probable relevance of this information is for practical structural condition assessment and damage detection based on continuous structural health monitoring data, where not every theoretical vibration mode of the structure is available, but rather one is forced to rely on the subset of modes which can be retrieved consistently from the monitoring data. It is observed that 8 out of 21 identified modes are consistently being excited regardless of loading variability while some modes; particularly at $0.55 \mathrm{~Hz}$ and $5.01 \mathrm{~Hz}$ are seldom excited .

Table 5-1 also shows information regarding the variability of the modal frequencies extracted from the monitoring datasets. In particular, SSI method offers a more consistent frequency estimate compared to other algorithms. The variation in the identified frequencies relative to the mean appears to be higher for the lower frequency vibration modes and shows a decreasing trend for higher frequency modes. This observation has very important significance since the lower vibration modes often represent the dominant vibration behaviour of most structures under typical dynamic loading conditions, and thus can impact on the use of vibration based algorithms for health monitoring purposes. 


\subsubsection{Mode shapes}

Modal Assurance Criteria (MAC) values between extracted and theoretical mode shapes are presented in Table 5-2. These values are computed between the mean of normalized extracted mode shapes and the corresponding theoretical mode shape. For the computation of the mean extracted mode shape, the mode shapes extracted from different datasets are normalized by their maximum coefficient. For all 4 algorithms, the average MAC value between extracted and theoretical mode shapes is greater than 0.80 , especially for SSI; the average MAC is 0.89 indicating very good correlation. ERA method, with an average MAC of 0.88 is as good as SSI at least in terms of mode shape estimation.

Figure 5-8 shows some of the important vibration mode shapes extracted by system identification process from the field monitoring data by SSI method and a comparison with the expected behaviour from the finite element model [73]. The overall agreement of the experimental results with the expected values is generally good, especially considering the complexity of the structure and with the use of output-only system identification methods in processing of ambient vibration data. As may be observed in Figure 5-8, at the expansion drop-in span, discrepancies between the identified and theoretical mode shape tend to be relatively larger. This fact stems probably from less perfect modeling of the actual drop-in span support condition. 


\subsubsection{Modal damping ratios}

The averages and standard deviations of the damping ratios of the vibration modes identified by different algorithms are presented in Table 5-2. In general, the standard deviations obtained for the damping ratios are much higher than those obtained for the modal frequencies especially for ERA and FDD methods; the high variance on damping estimate is quite noticeable. In case of FDD method, damping estimate for low frequency modes are unrealistically high, an indication of high leakage bias in spectra computation. In fact, the closeness of the modes and the relatively low frequency resolution adapted to guarantee significant number of averages which is essential for good performance of FDD technique, leads to an unaccepted biased spectra estimation. On the other hand, both SSI and PolyMAX methods provide comparable damping estimate. The average modal damping ratio by SSI method is $1.62 \%$ corresponding to $53 \%$ standard deviation of the mean while for PolyMAX; these values are $1.67 \%$ and $62 \%$ respectively. It is worth noting that the mean extracted modal damping ratios show a slight decreasing trend with increasing frequency. 
Table 5-1: Summary of modal frequencies identified by 4 algorithms and comparison to expected design values from finite element models

\begin{tabular}{|c|c|c|c|c|c|c|c|c|c|c|c|c|c|c|c|c|c|}
\hline \multirow[b]{2}{*}{ 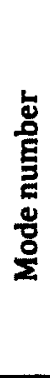 } & \multirow[b]{2}{*}{ 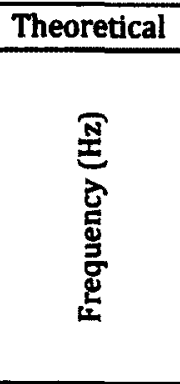 } & \multicolumn{4}{|c|}{ SSI } & \multicolumn{4}{|c|}{ PolyMAX } & \multicolumn{4}{|c|}{ ERA } & \multicolumn{4}{|c|}{ FDD } \\
\hline & & 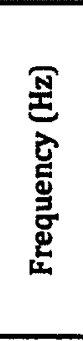 & 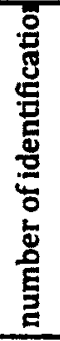 & 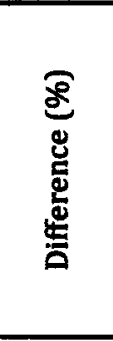 & $\underset{\mathscr{2}}{\stackrel{+}{8}}$ & 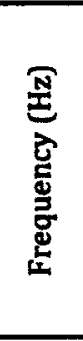 & 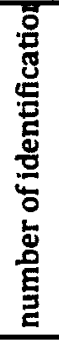 & 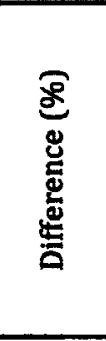 & $\begin{array}{l}\stackrel{+}{2} \\
\stackrel{2}{2} \\
\stackrel{2}{0}\end{array}$ & 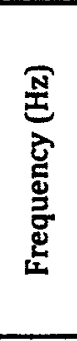 & 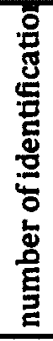 & 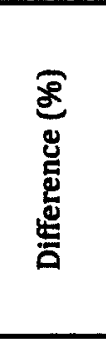 & $\frac{\hbar}{\stackrel{2}{2}}$ & 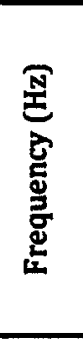 & 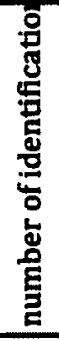 & 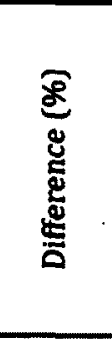 & $\frac{+}{\stackrel{5}{8}}$ \\
\hline 1 & 0.35 & $0.3 \overline{3}$ & 4 & -6.06 & 3.03 & 0.34 & 4 & -2.94 & 4.41 & 0.36 & 4 & 2.78 & 2.78 & 0.37 & 4 & 5.41 & 4.05 \\
\hline 2 & 0.54 & 0.48 & 14 & -12.50 & 0.10 & 0.47 & 14 & -14.89 & 2.13 & 0.48 & 14 & -12.50 & 0.21 & 0.48 & 14 & -12.50 & 2.08 \\
\hline 3 & 0.55 & 0.59 & 3 & 6.78 & 5.08 & 0.66 & 3 & 16.67 & 9.09 & --- & -- & -.. & $\ldots$ & $\cdots$ & $-\cdot$ & $\cdots$ & -- \\
\hline 4 & 0.69 & 0.66 & 11 & -4.55 & 1.52 & 0.67 & 11 & -2.99 & 2.69 & 0.65 & 11 & -6.15 & 3.08 & 0.68 & 11 & -1.47 & 6.62 \\
\hline 5 & 0.79 & 0.68 & 14 & -16.18 & 1.47 & 0.72 & 14 & -9.72 & 1.39 & 0.67 & 14 & -17.91 & 2.99 & 0.73 & 13 & -8.22 & 1.37 \\
\hline 6 & 0.84 & 0.92 & 2 & 8.70 & 1.09 & 0.91 & 4 & 7.69 & 1.10 & 0.91 & 3 & 7.69 & 2.20 & $-\cdot$ & --- & $\cdots$ & $\cdots$ \\
\hline 7 & 0.95 & 0.94 & 14 & -1.06 & 1.06 & 0.93 & 14 & -2.15 & 1.08 & 0.92 & 14 & -3.26 & 2.17 & 0.93 & 14 & -2.15 & 1.08 \\
\hline 8 & 0.98 & 0.97 & 8 & -1.03 & 1.10 & 0.96 & 8 & -2.08 & 1.04 & 0.96 & 8 & -2.08 & 2.08 & 0.99 & 8 & 1.01 & 1.14 \\
\hline 9 & 1.42 & 1.31 & 7 & -8.40 & 1.53 & 1.33 & 7 & -6.77 & 0.75 & 1.35 & 7 & -5.19 & 2.12 & 1.29 & 7 & -10.08 & 1.63 \\
\hline 11 & 1.82 & 1.81 & 14 & -0.55 & 1.10 & 1.85 & 14 & 1.62 & 0.54 & 1.82 & 14 & 0.00 & 0.55 & 1.82 & 14 & 0.00 & 2.20 \\
\hline 12 & 2.46 & 2.77 & 14 & 11.19 & 0.72 & 2.80 & 14 & 12.14 & 0.36 & 2.77 & 12 & 11.19 & 0.36 & 2.79 & 14 & 11.83 & 1.43 \\
\hline 13 & 2.92 & 2.95 & 8 & 1.02 & 0.68 & 2.91 & 8 & -0.34 & 0.34 & 2.93 & 8 & 0.34 & 0.34 & 2.87 & 8 & -1.74 & 1.39 \\
\hline 14 & 3.17 & 3.12 & 7 & -1.60 & 0.64 & 3.15 & 7 & -0.63 & 0.48 & 3.13 & 7 & -1.28 & 0.80 & 3.11 & 7 & -1.93 & 0.90 \\
\hline 15 & 3.51 & 3.31 & 4 & -6.04 & 0.90 & 3.35 & 5 & -4.78 & 0.54 & 3.29 & 3 & -6.69 & 0.61 & 3.3 & 4 & -6.36 & 0.96 \\
\hline 16 & 3.15 & 3.45 & 14 & 8.70 & 0.58 & 3.42 & 14 & 7.89 & 0.88 & 3.42 & 14 & 7.89 & 0.58 & 3.4 & 14 & 7.35 & 0.77 \\
\hline 17 & 3.6 & 3.79 & 7 & 5.01 & 0.60 & 3.68 & 6 & 2.08 & 0.68 & 3.82 & 7 & 5.76 & 0.52 & 3.73 & 9 & 3.49 & 0.64 \\
\hline 18 & 4.01 & 4.15 & 4 & 3.37 & 0.10 & 4.14 & 3 & 3.14 & 0.48 & 4.16 & 6 & 3.61 & 0.13 & 4.16 & 2 & 3.61 & 0.48 \\
\hline 19 & 4.43 & 4.69 & 7 & 5.54 & 0.68 & 4.66 & 7 & 4.94 & 0.43 & 4.63 & 7 & 4.32 & 0.57 & 4.86 & 5 & 8.85 & 0.41 \\
\hline 20 & 5.01 & 4.91 & 3 & -2.04 & 0.81 & 4.88 & 3 & -2.66 & 1.16 & 4.92 & 3 & -1.83 & 1.02 & 4.96 & 3 & -1.01 & 1.21 \\
\hline 21 & $\cdots$ & 5.18 & 8 & $\ldots$ & 0.58 & 5.18 & 8 & $\ldots$ & 0.39 & 5.1 & 8 & -.. & 0.25 & 5.14 & 6 & -- & 0.39 \\
\hline & Averages & & 9 & -0.39 & 1.17 & & 9 & 0.43 & 1.46 & & 9 & -0.57 & 1.25 & & 9 & -0.15 & 1.64 \\
\hline
\end{tabular}

† Relative Standard deviation 
Table 5-2: Summary of modal damping ratios and Modal Assurance Criterion values for vibration modes retrieved by 4 system identification algorithms

\begin{tabular}{|c|c|c|c|c|c|c|c|c|c|c|c|c|c|}
\hline \multirow[b]{2}{*}{ 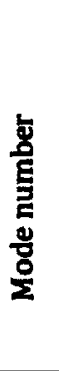 } & \multirow[b]{2}{*}{ Theoretical } & \multicolumn{3}{|c|}{ SSI } & \multicolumn{3}{|c|}{ PolyMAX } & \multicolumn{3}{|c|}{ ERA } & \multicolumn{3}{|c|}{ FDD } \\
\hline & & 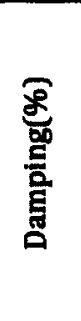 & 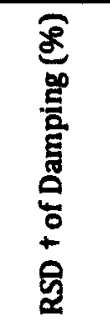 & 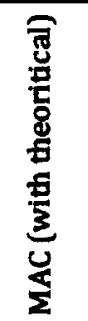 & 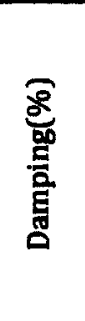 & 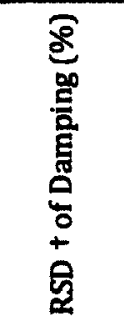 & 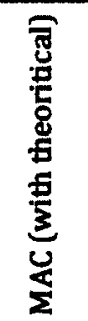 & 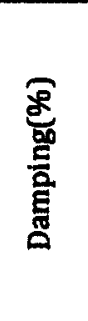 & 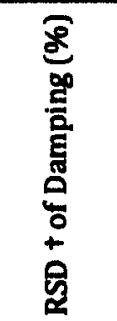 & 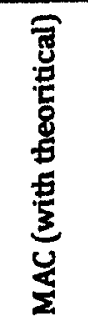 & 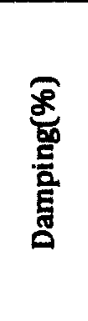 & 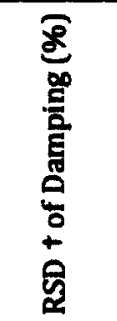 & 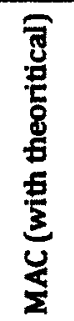 \\
\hline 1 & 0.35 & 1.90 & 50.53 & 0.92 & 4.10 & 48.29 & 0.59 & 4.86 & 123.46 & 0.86 & 4.30 & 118.60 & 0.85 \\
\hline 2 & 0.54 & 1.69 & 56.80 & 0.87 & 1.76 & 39.77 & 0.84 & 2.49 & 116.47 & 0.89 & 8.00 & 187.50 & 0.84 \\
\hline 3 & 0.55 & 2.94 & 32.65 & 0.91 & 2.76 & 29.93 & 0.73 & --- & $\ldots$ & $\ldots$ & - & $\ldots$ & $\ldots$ \\
\hline 4 & 0.69 & 1.85 & 51.89 & 0.98 & 1.81 & 48.87 & 0.84 & 4.39 & 71.53 & 0.98 & 4.00 & 106.25 & 0.87 \\
\hline 5 & 0.79 & 2.10 & 45.71 & 0.78 & 2.03 & 47.01 & 0.83 & 4.55 & 153.41 & 0.88 & 4.99 & 100.20 & 0.79 \\
\hline 6 & 0.84 & 1.50 & 64.00 & 0.84 & 1.43 & 61.14 & 0.84 & 2.70 & 78.52 & 0.80 & $\ldots$ & $\ldots$ & $\ldots$ \\
\hline 7 & 0.95 & 2.26 & 42.48 & 0.97 & 1.98 & 39.90 & 0.84 & 2.43 & 91.77 & 0.89 & 2.10 & 94.29 & 0.85 \\
\hline 8 & 0.98 & 1.60 & 43.75 & 0.89 & 1.01 & 154.46 & 0.48 & 1.54 & 87.66 & 0.89 & 2.30 & 104.35 & 0.77 \\
\hline 9 & 1.42 & 0.70 & 114.29 & 0.80 & 0.92 & 97.83 & 0.84 & 0.88 & 90.91 & 0.85 & 5.10 & 141.18 & 0.79 \\
\hline 10 & 1.6 & 2.30 & 33.91 & 0.93 & 2.01 & 105.97 & 0.83 & 1.40 & 171.43 & 0.90 & 3.30 & 93.94 & 0.83 \\
\hline 11 & 1.82 & 1.90 & 51.58 & 0.95 & 1.82 & 49.18 & 0.83 & 1.82 & 103.30 & 0.87 & 1.10 & 89.09 & 0.89 \\
\hline 12 & 2.46 & 1.37 & 62.77 & 0.93 & 1.42 & 61.97 & 0.84 & 1.89 & 103.17 & 0.89 & 1.70 & 82.35 & 0.81 \\
\hline 13 & 2.92 & 1.82 & 48.90 & 0.89 & 1.90 & 48.42 & 0.83 & 1.37 & 89.78 & 0.89 & 1.90 & 47.37 & 0.83 \\
\hline 14 & 3.17 & 1.25 & 72.00 & 0.95 & 1.33 & 69.17 & 0.86 & 1.52 & 88.82 & 0.86 & 1.20 & 95.00 & 0.82 \\
\hline 15 & 3.51 & 0.92 & 43.48 & 0.79 & 0.79 & 112.66 & 0.72 & 1.06 & 99.06 & 0.88 & 1.23 & 79.67 & 0.79 \\
\hline 16 & 3.15 & 1.27 & 42.52 & 0.78 & 1.32 & 37.12 & 0.85 & 2.21 & 95.02 & 0.88 & 2.00 & 75.00 & 0.55 \\
\hline 17 & 3.6 & 0.90 & 55.56 & 0.87 & 0.98 & 79.59 & 0.81 & 2.05 & 58.54 & 0.86 & 1.34 & 85.82 & 0.77 \\
\hline 18 & 4.01 & 0.97 & 80.00 & 0.86 & 0.93 & 19.35 & 0.82 & 1.13 & 86.73 & 0.81 & 1.00 & 97.00 & 0.90 \\
\hline 19 & 4.43 & 2.30 & 30.87 & 0.93 & 2.09 & 35.50 & 0.84 & 1.55 & 38.71 & 0.87 & 1.00 & 110.00 & 0.68 \\
\hline 20 & 5.01 & 1.01 & 67.33 & 0.95 & 0.97 & 63.92 & 0.88 & 1.08 & 75.00 & 0.92 & 1.16 & 113.79 & 0.87 \\
\hline 21 & - & 1.55 & 32.26 & - & 1.77 & 45.08 & $\ldots$ & 2.06 & 31.55 & - & 2.25 & 115.56 & $\ldots$ \\
\hline & Averages & 1.62 & 53.00 & 0.89 & 1.67 & 62.00 & 0.80 & 2.15 & 93.00 & 0.88 & 2.63 & 102.00 & 0.81 \\
\hline
\end{tabular}



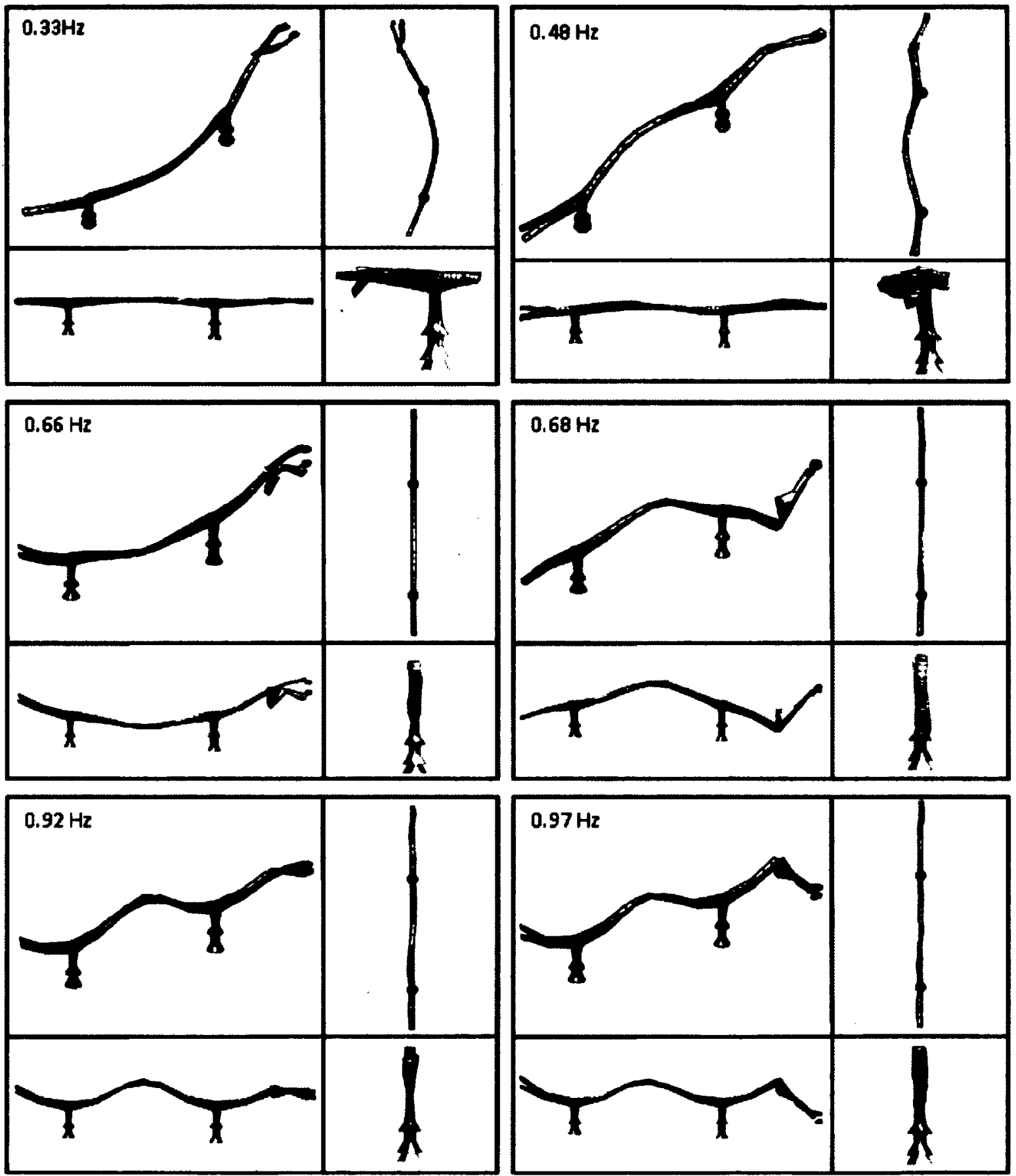

Figure 5-8(a): Comparison of experimental and analytical mode shapes between $0 \mathrm{~Hz}$ to 1 Hz. Vibration modes from the finite element model are shown in green with doted lines and the modes identified from the monitoring data are shown in grey with full lines [72]. 

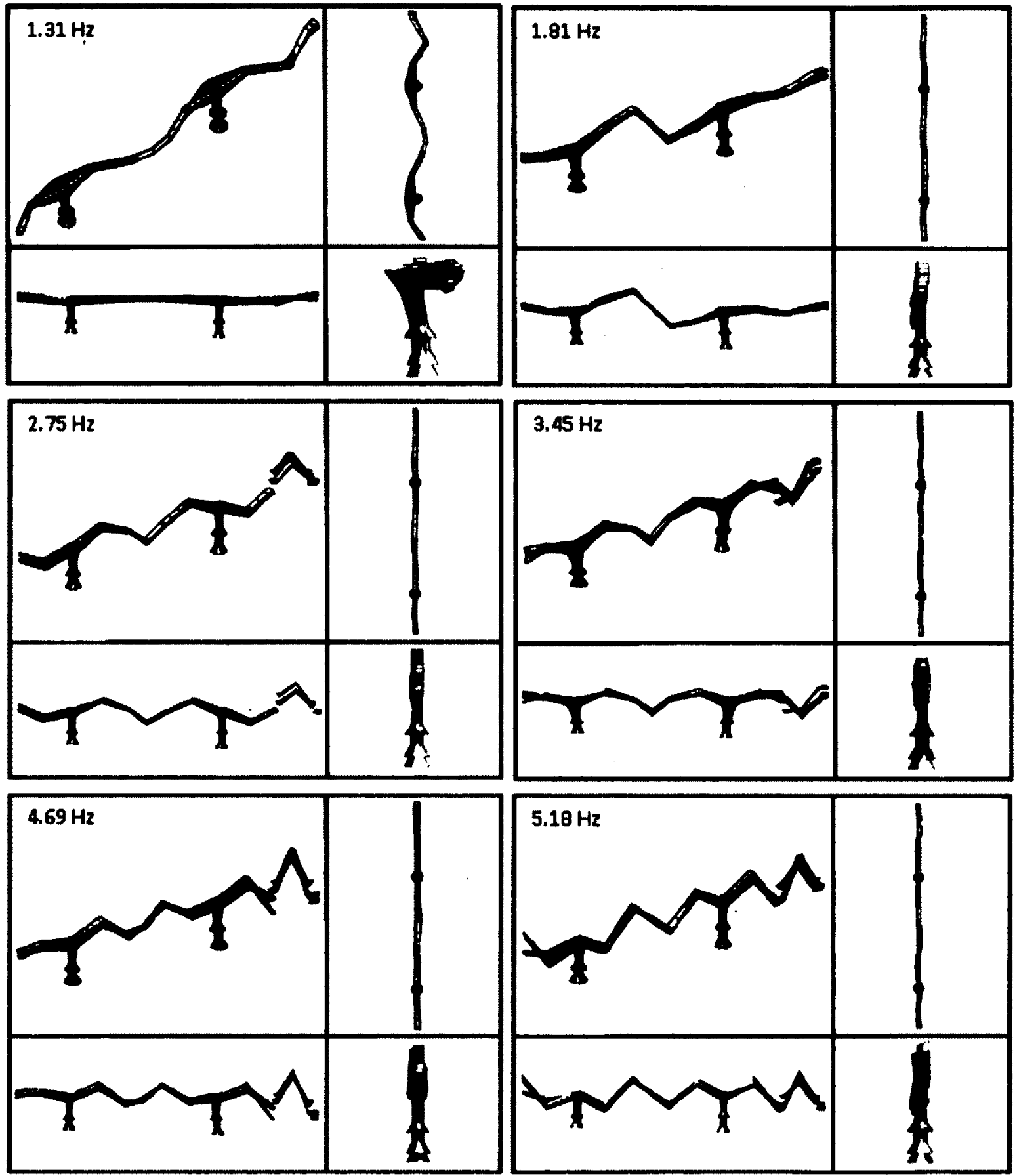

Figure 5-8(b): Comparison of some experimental and analytical mode shapes above $1 \mathrm{~Hz}$. Vibration modes from the finite element model are shown in green with doted lines and the modes identified from the monitoring data are shown in grey with full lines [72]. 


\subsection{Conclusion}

In the present study, a total of fourteen field monitoring datasets from the Confederation Bridge monitoring database have been analyzed by four different output-only system identification techniques and the extracted modal properties are investigated against expected design values.

From the output only system identification analysis of the monitoring data, twenty one vibration modes are identified in the in the frequency range $0-5.5 \mathrm{~Hz}$, exhibiting an overall very good correlation with the modal properties previously calculated using the design finite element modeling. The identified modal frequencies differ by less than $1 \%$ on average from the expected design values and the extracted mode shapes are reasonably similar to those expected from the design except for some localized discrepancies at the drop-in-span where the actual asymmetrical support conditions are more complex than those assumed in the finite element models of this study.

Among all the methods, SSI-COV algorithm seems to be more consistent in frequency, damping and mode shape estimate. The application of PolyMAX and SSICOV methods to the ambient vibration data results in a very similar estimates for natural frequencies and damping ratios while for mode shape estimation; SSI-COV outperforms PolyMAX method. The more traditional identification algorithm, ERA method provides comparable results at least in terms of frequency and mode shape 
estimation. Furthermore, all four algorithms exhibit higher variance in damping estimate especially for ERA and FDD method, it is quite noticeable. 


\section{Chapter 6 - Conclusions and}

\section{Recommendations for Future}

\section{Work}

\subsection{Summary of findings}

Despite being a relatively new field, Operational Modal Analysis (OMA) has garnered significant attention in the research community and become more popular with time especially in situations, difficult to handle using traditional Experimental Modal Analysis (EMA) techniques. However, the field is still in its developmental stage and efforts are needed towards understanding its advantages and limitations in more detail. The purpose of this study is to evaluate the efficiency and accuracy of different modal identification methods, particularly in the presence of high level of uncertainty and noise related to field measurement data. The work presented in this dissertation can be divided in two aspects as follows 
- Illustration of existing OMA parameter estimation techniques and associated signal processing.

- Evaluation the performance of existing OMA parameter estimation techniques with the Confederation Bridge monitoring data and assessment of conditions where the OMA parameter estimation process work satisfactorily and where it will face difficulty, thus providing constructive insights to the application of OMA techniques.

The salient features, based on the research carried out in the dissertation, can be summarized as follows.

- 4 state-of-the-art stochastic system identification methods - FrequencyDomain Decomposition (FDD), Eigensystem Realization Algorithm (ERA), Stochastic Subspace Identification (SSI) and the PolyMAX method are compared with a simulated 8-DOF example and with the Confederation Bridge monitoring data. From the comparison of the estimated modal parameters, it is concluded that the subspace methods are the preferred methods, especially the SSI method is more consistent in estimating modal parameter among all the methods. None of the methods are robust against less excited modes. Additionally, mode shape estimation by the PolyMAX method is the victim of comparatively high bias and variance due to biased correlogram spectrum estimation.

- Output response power spectra, which are comparatively more difficult to estimate than the frequency response functions, greatly affects the modal 
damping estimation due to the leakage errors. For all the methods, the effect of bias and variance on damping estimate is quite evident; especially for the FDD method the effect is severe. Cyclic Averaging is a powerful signal processing technique which should be used along with windowing and overlapping for reducing the effects of leakage and hence improving the accuracy of damping estimates though judgment should be employed in selecting number of cyclic averaging. On the hand, use of an exponential window with a reasonably long lag time significantly reduces the leakage error in spectrum estimation and thus provides a better damping estimate.

- The accuracy of damping estimates is also affected in situations where the basic OMA assumption of uncorrelated and broadband excitation force is violated. While the leakage related issues in spectrum estimation can be dealt with, up to a certain extent by using techniques like Cyclic Averaging and exponential windowing, the nature of ambient excitation is beyond one's control and thus difficult to deal with in real life situations.

- Operational Modal Analysis (OMA) assumes a zero mean Gaussian white noise input. If the input contains dominant frequency components in addition to the white noise, then those frequency components cannot be separated from the eigenfrequencies of the system.

- Most of the modal identification algorithms presented in the thesis require pre-selection of model order, and frequently over determination of model order generates a lot of spurious poles. For random response data like in 
OMA application, the problem becomes more acute. Use of stabilization diagram to distinguish pure modes from the noisy ones is an effective measure but still the modal estimation process influenced to a great extent by the expertise of the analyst.

\subsection{Future work}

Based on the current investigation, the writer believes that the following issues merit future investigation:

- The damping estimation is one area which needs to be explored further to understand and identify the reasons behind inaccurate damping estimation in OMA application. Future research should concentrate on finding signal processing techniques for more precise estimation of damping especially in the case of real life application where the input excitation is correlated and input spectrum is not constant.

- A very new trend in OMA application is to employ signal processing techniques other than power spectra or correlation functions as primary identification data. Some of these attempts include exploitation of transmissibility functions [74], cyclostationarity and cepstrum analysis [75] etc. It will be interesting to investigate whether these methods yield more accurate damping estimates as they utilize different signal processing approach. And at the same time, the performance of these new algorithms 
should be compared with more established OMA algorithms while testing real structures.

- Adopt newer and more computationally intensive numerical system identification techniques which can deal with non-linearity and nonstationarity of the structure and its data. One such algorithm would be the Hilbert-Huang empirical method [76], [77], [78]. 


\section{References}

[1] Jer-Nan Juang, Applied System Identification. Upper Saddle River: Prentice Hall, 1994.

[2] N.M. Silva and Maia J.M., Theoretical and Experimental Modal Analylis.: John Wiley \& Sons, 1997.

[3] W, Heylen, S. Lammens, and P. Sas, Modal analysis theory and testing. KU. Leuven, Belgium, 1997.

[4] R.J. Allemang. (1999) Vibrations: Experimental Modal Analysis Course Notes.Seventh edition, Structural Dynamics Research Laboratory, University of Cincinnati, $\mathrm{OH}, \quad$ USA. [Online]. http://www.sdrl.uc.edu/academic-course-info/vibrations-iii-20-263$\underline{663}$

[5] T. Kailath, Linear systems. New Jersey: Prentice-Hall, 1980.

[6] Guillaume P., Pintelon R., and Schoukens J., "Parametric identification of multivariable systems in the frequency domain - a survey," in 21st International Seminar on Modal Analysis, Belgium, 1996. 
References

[7] H. Akaike, "Markovian representation of stochastic processes and its application to the analysis of autoregressive moving average processes," Anuals of the Institute of Statistical Mathematics, vol. 26, pp. 363-387, 1974.

[8] F. Lembregts, "Frequency domain identification for experimental multiple inputs modal analysis," Ph.D thesis, Department of Mechanical Engineering, Katholieke University, Leuven, Belgium, 1988.

[9] Bart Cauberghe, "Applied frequency-domain system identification in the field of experimental and operational modal analysis," Ph.d Thesis, Department of Mechanical Engineering, Vrije University, Brussel, 2004.

[10] T Soderstrom and P Stoica, System Identification. UK: Prentice Hall, 1989.

[11] P Van Overschee and Br De Moor, "Subspace algorithms for the stochastic identification problem," Automatica, vol. 29, no. 3, pp. 649-660, 1993.

[12] L Ljung, System Identification: Theory for the User.: Prentice-Hall, 1999.

[13] R Pintelon and J Schoukens, System identification: A frequency domain approach.: IEEE Press, 2001.

[14] B. L. Ho and R. E. Kalman, "Effective construction of linear state-variable models from input/output data," Regelungstechnik, vol. 14, no. 12, pp. 545-548, 1966.

[15] B Peeters, "System identification and damage detection in civil engineering," PhD thesis, Department of Civil Engineering, KU, Leuven, Belgium, 2000. 
References

[16] B. Peeters and G De Roeck, "Reference Based Stochastic Subspace Identification for Output-Only Modal Analysis," Mechanical System and Signal Processing, vol. 13, no. 6, pp. 855-878, 1999.

[17] S.L. Marple, Digital Spectral Analysis with Applications. Englewood Cliffs: Prentice-Hall Inc., 1987.

[18] E. Parloo, "Application of frequency-domain system identification techniques in the field of operational modal analysis," PhD Thesis, Department of Mechanical Engineering, V.U. Brussel, Belgium, 2003.

[19] L. Hermans, H. Van der Auweraer, and P. Guillaume, "A frequency-domain maximum likelihood approach for the extraction of modal parameters from output-only data," in Proceedings of ISMA23, the International Conference on Noise and Vibration Engineering, 1998, pp. 367-376.

[20] S.R. Ibrahim and E.C. Mikulcik, "A time domain modal vibration test technique," Shock and Vibration Bulletin, vol. 43, no. 4, pp. 21-37, 1973.

[21] H. Vold, J. Kundrat, T. Rocklin, and R. Russell, "A Multi-Input Modal Estimation Algorithm for Mini-Computers," SAE Paper Number 820194, 1982.

[22] J.N. Juang and R.S. Pappa, "An eigen system realization algorithm for modal parameter identification and model reduction," Journal of Guidance, Control and Dynamics, vol. 8, no. 5, pp. 620-627, 1985.

[23] R.J. Allemang and D.L Brown, "A unified matrix polynomial aprroach modal identification," Journal of Sound and Vibration, vol. 211, no. 3, pp. 301- 
$\underline{\text { References }}$

$322,1998$.

[24] J., Piersol Bendat, Random Data: Analysis and Measurement Procedures 2nd Edition. New York: Wiley, 1986.

[25] C.Y. Shih, Y.G. Tsuei, R.J. Allemang, and D.L. Brown, "Complex Mode Indication Function and Its Application to Spatial Domain Parameter Estimation," Mechanical System and Signal Processing, vol. 2, no. 4, pp. 367-377, 1988.

[26] J N Juang and Phan Minh., Identification and Control of Mechanical System. UK: Cambridge University Press, Cambridge, 2006.

[27] W. Gersch and D.A. Fouth, "Least Squares Estimates of Structural System," IEEE Transactions on Automatic Control, vol. 19, no. 6, pp. 898-903, 1974.

[28] S.M. Pandit, "Analys is of Vibration Records by Data Dependent Systems," Shock and Vibration Bulletin, Number 47, pp. 161-174, 1977.

[29] W Gersch and S Luo, "Discrete Time Series Synthesis of Randomly Excited Structural System Responses," Journal of the Acoustical Society of America, vol. 51, no. 1B, pp. 402-408, 1972.

[30] G.H. James, T.G. Carne, and Lau J.P., "The natural excitation technique for modal parameter extraction from operating strucutres," Modal analysis, vol. 10, no. 4, pp. 260-277, 1995.

[31] P. Andersen, "Identification of Civil Engineering Structures using Vector," Ph.D. Thesis, Aalborg University, Denmark, 1997. 
References

[32] B. Peeters and G. De Roeck, "Stochastic System Identification for Operational Modal Analysis: A Review," Journal of Dynamic Systems,Measurement and Control, vol. 123, no. 4, pp. 659-667, 2001.

[33] H.P. Zeiger and A.J. McEwen, "Approximate linear realization of given dimension via Ho's algorithm," IEEE Transactions on Automatic Control, vol. 19 , no. 2 , p. $153,1974$.

[34] S. Kung, "New Identification and Model Reduction Algorithm via Singular Value Decomposition," in Proceedings of the 12th Asilomar Conference on Circuits, System and Computers, Asilomar, CA, USA, 1978, pp. 705-714.

[35] N. Londono, "Use of vibration data for structural health monitoring of bridges," PhD thesis, Department of Civil \& Environmental Engineering, Carleton University, Ottawa, Canada, 2006.

[36] P Van Overschee and B De Moor, Subspace Identification for Linear Systems: Theory-Implemantation-Applications. Dordrecht, Netherlands: Kluwer Academic Publishers, 1996.

[37] R Brincker, L. Zhang, and P. Andersen, "Modal identification from ambient response susing frequency domain decomposition," in Proceedings of the IMAC 18 18, International Modal Analysis Conference, SanAntonio, USA, 2000.

[38] P. Welch, "The use of fast fourier transform for the estimation of power spectra: a method based on time averaging over short, modified periodograms," 
References

IEE Transactions on Audio and Electroacoustics, vol. 15, no. 2, pp. 70-73, 1967.

[39] R.J. Allemang, "Investigation of some multiple input/output frequency response function experimental modal analysis techniques," Ph.d Thesis, University of Cincinnati, Mechanical Engineering Department, Cincinnati, USA, 1980.

[40] S. Chauhan, R. Martell, R. J. Allemang, and D. L Brown, "A Low Order Frequency Domain Algorithm for Operational Modal Analysis," in Proceedings of ISMA International Conference on Noise and Vibration Engineering, 2006.

[41] P. Guillaume, P. Verboven, and S. Vanlanduit, "Frequency domain maximul likelihood identification of modal parameters with confidence intervals," in Proceedings of ISAM 23, Noise and Vibration Engineering , 1998.

[42] H. Van Der Auweraer, P. Guillaume, P. Verboven, and S. Vanlanduit, "Application of a fast stabilizing frequency domain parameter estimation method," ASME Journal of Dynamic System, Measurement and Control, vol. 123, no. 4, pp. 651-658, 2001.

[43] P. Verboven, "Frequency-domain system identification for modal analysis," PhD thesis, Department of Mechanical Engineering, V.U. Brussel, Belgium, 2002.

[44] P Guillaume, P Verboven, S. Vanlanduit, H. Van Der Auweraer, and Bart Peeters, "A polyreference implementation of the least square complex frequency 
domain estimator," in Proceedings of the IMAC 21, International Modal Aanlysis Conference, Kissimmee, Florida, USA, 2003.

[45] B Peeters et al., "Automative and aerospace applicaiton of PolyMAX modal parameter estimation method," in Proceedings of IMAC 22, the Internaitonal Modal Analysis Conference, Dearborn,MI, USA, 2004.

[46] B. Peeters, A. Vecchio, and H. Van der Auweraer, "PolyMAX modal parameter estimation from operational data," Proceedings of the 2004 International Conference on Noise and Vibration Engineering, pp. 1049-1063, 2004b.

[47] B. Cauberghe, P. Guillaume, P Verboven, S. Vanlanduit, and E. Parloo, "On the influence of the parameter constraint on the stability of the poles and the discrimination capabilities of the stabilisation diagrams," Mechanical Systems and Signal Processing, vol. 10, no. 5, pp. 989-1014, 2005.

[48] ]. Schoukens and R. Pintelon, Identification of Linear Systems: a Practical Guideline to Accurate Modelling. London, UK: Pergamon Press, 1991.

[49] R. Pintelon, P. Guillaume, Y. Rolain, J. Schoukens, and Van Hamme H., "Parametric identification of transfer functions in the frequency domain - a survey," IEEE Transactions on Automatic Control, vol. 39, no. 11, pp. 2245-2260, 1994.

[50] J.S. Bendat and A.G. Piersol, Engineering Applications of Correlation and Spectral Analysis. New York: John Wiley, 1980.

[51] P. Andersen et al., "Comparison of systems identification methods using 
References

ambient bridge test," in Proceedings of the 17th IMAC, International Modal Analysis Conference, Kissimmee, Florida, 1999.

[52] B. Peeters and Carlos Ventura, "Comparative study of modal analysis techniques for bridge dynamic characteristics," Mechanical Systems and Signal Processing, vol. 15, no. 5, pp. 965-988, 2003.

[53] T. Horyna and C. Ventura, "Summary of HCT Building Ambient Vibration Data Analysis," in Proceedings of the 18th IMAC, International Modal Analysis Conference, San Antonio, TX, USA, 2000, pp. 1095-1098.

[54] A. Cunha, E. Caetano, R. Brincker, and P. Andersen, "Identification from the Natural Response of the Vasco Da Gama Bridge," in Proceedings of the 22nd International Modal Analysis Conference (IMAC), Detroit, Michigan, 2004, pp. 202-209.

[55] L. Hermans and H. Van der Auweraer, "Modal Testing and Analysis of Structures Under Operationa Conditions: Industrial Applicatoins," Mechanical System and Signal Processing, vol. 13, no. 2, pp. 193-216, 1999.

[56] A. Vecchio, B. Peeters, and H. Van der Auweraer, "Application of Advanced Parameter Estimators to the Analysis of In-Flight Measured Data," in Proceedings of 20th IMAC, International Modal Analysis Conferene, LA, USA, 2002.

[57] B. Peeters, H. Van der Auweraer, F. Vanhollebeke, and P. Guillaume, 
"Operational modal analysis for estimating the dynamic properties of a stadium structure during a football game," Shock and Vibration, vol. 14, no. 4, pp. 283-303, 2007.

[58] L. Mevel, M. Basseville, A. Benveniste, and M. Goursat, "Merging sensor data from multiple measurement set-ups for non-stationary subspace-based modal analysis," Journal of Sound and Vibraiton, vol. 249, no. 4, pp. 719 741., 2002.

[59] N.J. Jacobsen, "Separating Structural Modes and Harmonic Components in Operational Modal Analysis," in Proceedings of the 24th IMAC, Internaitonal Modal Analysis Conference, St. Louis, MO, USA, 2006.

[60] Shashank Chauhan, "Parameter Estimation and Signal Processing Techniques for Operational Modal Analysis," Ph.d theis, Department of Mechanical Engineering, University of Cincinnati, Cincinnati, Ohio, USA, 2008.

[61] D Bernal and B. Gunes, "Damage Localization in Output-Only systems: A Flexibility Based approach," in Proceedings of the International Modal Analysis Conference (IMAC) XX, Los Angles, CA, USA, 2002.

[62] Diego F. Giraldo, Wei Song, Shirley J. Dyke, and Juan M. Caicedo, "Modal Identification through Ambient Vibration: Comparative Study," Journal of Engineering Mechanics, vol. 135, no. 8, pp. 759-770, 2009.

[63] Ryan Oconnell, "Advances in intelligent continuous structural helath monitoring of bridges," Maters thesis, Department of Civil \& 
References

vol. 9, no. 1, pp. 141-157, 2006.

[71] S. Desjardins, "Real-time computer platform for vibration-based structural health monitoring of the Confederation Bridge," Maters thesis, Department of Civil \& Environmental Engineering, Carleton University, Ottawa, Canada, 2004.

[72] N.A. Londono, D.T. Lau, and M. Rahman, "Characteristics of dynamic monitoirng data and observed behaviour of the Confederation Bridge due to operational load variations," Canadian Journal of Civil Engineering, in review.

[73] D.T. Lau, T Brown, Cheung M.S., and W.C. Li, "Dynamic modelling and behaviour of the Confederaion Bridge," Canadian Journal of Civil Engineering, vol. 31, no. 2, pp. 379-390, 2004.

[74] C. Devriendt, P. Guillaume, E. Reynders, and G. De Roeck, "Operational Modal Analysis of a Bridge Using Transmissibility Measurements," in Proceedings of the 25th IMAC, Orlando (FL), USA, 2007.

[75] D. Hanson et al., "Cyclostationary and the Cepstrum for Operational Modal Analysis of MIMO Systems- Part I: Modal Parameter Identification," Mechanical Systems and Signal Processing, vol. 21, no. 6, pp. 2441-2458, 2007.

[76] N.E. Huang et al., "The Empirical Mode Decomposition and the Hilbert Spectrum for Nonlinear and Non-Stationary Time Series Analysis," vol. 
References

454, no. 1971, pp. 903-995, 1998.

[77] J.N. Yang, Y. Lei, S. Pan, and N. Huang, "System identification of linear structures based on Hilbert-Huang spectral analysis. Part 1 : Normal modes," Journal of Earthquake Engineering and Structural Dymamics, vol. 32, no. 9, pp. 1443-1467, 2003.

[78] J.N. Yang, Y. Lei, S. Pan, and N. Huang, "System identification of linear structures based on Hilbert-Huang spectral analysis. Part 2 : Complex modes," Journal of Earthquake Engineering and Structural Dymamics, vol. 32, no. 10, pp. 1533-1554., 2003. 